\title{
AN EXPLORATION OF THEORIES OF ACTION IN LEADERSHIP DEVELOPMENT: A CASE STUDY
}

Scott J. Allen

\author{
A DISSERTATION \\ Submitted to the Ph.D. in Leadership \& Change Program \\ of Antioch University \\ in partial fulfillment \\ of the requirements for the degree of \\ Doctor of Philosophy
}




\section{SIGNATURE PAGE}

This is to certify that the dissertation entitled

\section{AN EXPLORATION OF THEORIES OF ACTION IN LEADERSHIP DEVELOPMENT: A CASE STUDY}

Prepared by

Scott J. Allen

is approved in partial fulfillment of the requirements for the degree of Doctor of Philosophy in Leadership and Change.

Approved by:

Chair - Jon Wergin, Ph.D.

Date

Committee Member - Richard Couto, Ph.D.

Date

Committee Member, Jim Crawford, Ph.D.

Date

External Reader, Cynthia McCauley, Ph.D.

Date 
Copyright 2006

Scott J. Allen

All rights reserved 


\begin{abstract}
The central purpose of the present study was to examine how academic notions of leadership development compare and contrast with the theory of action that guides corporate leadership development initiatives. A secondary purpose was to analyze the process and potential extensions of the user-focused theory of action approach.

Initial findings suggest that the user-focused theory of action approach is transferable to the case studied. In addition, an analysis of the leadership development literature and the Frontline Leadership Excellence System yielded a thought-provoking comparison of theory and practice. The study also provided an analysis of literature gaps and useful suggestions regarding the user-focused theory of action process and extensions for practice.
\end{abstract}




\section{ACKNOWLEDGEMENTS}

I would like to acknowledge the following people, places and things for their support and guidance:

My wife Jessica for her endless love and support.

Dr. Jon Wergin for being a wonderful guide and mentor throughout the dissertation process.

Dr. Richard Couto for his advice and direction throughout the Ph.D. program.

Dr. Jim Crawford for his enthusiasm and support throughout.

Dr. Cynthia McCauley — a mentor from afar.

Dr. Brenda Gardner and Dr. Sharon Korth for encouraging me to pursue a Ph.D. following graduate school.

Jack and David Allen for their support in making this research possible.

All participants who gave their time: Lynn, Oprah and Ms. Reeves.

Steve Becker for his thoughtful feedback.

Cohort Two.

Phoenix Coffee Shop.

The branch of philosophy that studies the nature of knowledge, its presuppositions and foundations, and its extent and validity. 


\section{DEDICATION}

This study is dedicated to my grandparents:

Wilmer and Shirley Kirkeby

Maxine and Harold Allen

The greatest use of a life is to spend it on something that will outlast it.

- William James 


\section{TABLE OF CONTENTS}

Title Page........................................................1

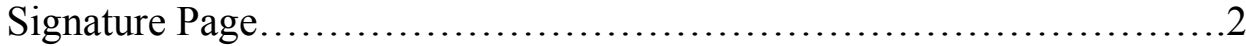

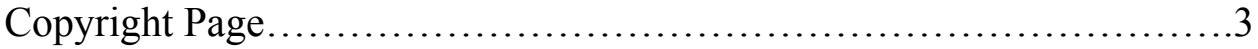

Abstract........................................................... 4

Acknowledgments............................................... 5

Dedication.......................................................

Table of Contents.................................................. 7

CHAPTER

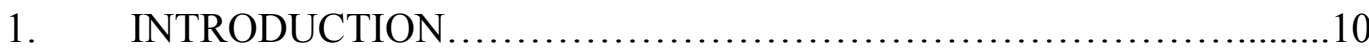

Challenges of Leadership Development........................11

The Theoretical Framework....................................16

Definitions................................................. 18

Study Design..........................................22

Limitations.............................................23

Delimitations - Boundaries...............................23

Assumptions...............................................23

Advance Organizer......................................24

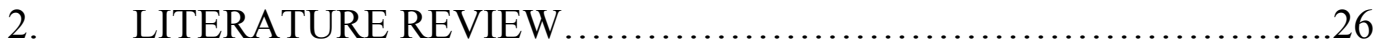

The Leadership Development Landscape.......................26

Limitations of the Leadership Development Literature.............29

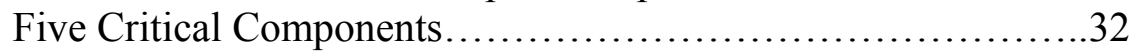

Leadership Theory..................................32

The Organizational Context............................34

Adult Development and Learning Theory................45

Development Tools..................................62

Evaluation..........................................83

Chapter Two Summary.................................. 92

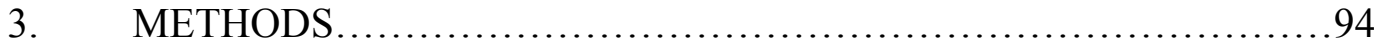

Situating Myself in the Research............................99

Case Study Methodology.....................................96

User-Focused Theory of Action Approach.......................100

The Pilot Study.............................................103

Primary Study - Procedures...............................109

Issues of Validity, Reliability and Generalizability...............117

Ethical Issues............................................119

Chapter Three Summary..................................120

4. DATA COLLECTION AND RESULTS ............................122 
The Beta Company Case Study ...............................123

Background Information..............................123

Current Organization Structure..........................127

Leadership Excellence and Organizational Research.....129

Background on the FLES.............................131

Identified Components of the FLES...................132

Development Tools....................................135

Beta Company's FLES Timeline.........................141

The Beta Company Theory of Action............................142

The Theory of Action Explained...........................148

Results, Reactions, and Findings - The Theory of Action ........157

Making Explicit the Theory of Action .....................157

The Process Sparked a Lively Conversation...............158

Multiple Levels of Discussion...........................160

The Process Sparked Informal Brainstorming.............161

Theory of Action as a Diagnostic Tool.....................161

Potential for Resistance, Defensiveness \& Frustration...163

No New Information....................................164

Discussion Points from the Literature.............................166

Leadership Theory ...................................168

The Organizational Context............................169

Adult Development and Learning........................170

Development Tools..................................171

Evaluation......................................176

Chapter Four Summary .................................... 179

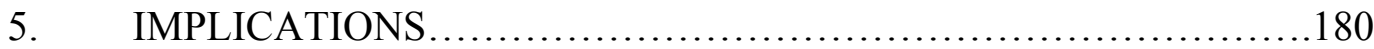

The Literature on Leadership Development...................181

Marketing/Communication...........................182

Implementation......................................183

The User-Focused Theory of Action Process.......................185

Organizational Awareness...............................186

Semi-Structured Interview Process.......................187

Adaptation of Patton's Original Process...................188

A Symbiotic Process of Theory Development.............190

Participant Ownership..................................191

Knowledge of Leadership Literature......................191

Time......................................................192

Willing and Open Participants..........................192

The User-Focused Theory of Action - Extensions for Use.......193

Developing a Realistic Theory of Action..................195

As a Planning Tool....................................196

As a Diagnostic Tool..................................198

A Springboard for Brainstorming.......................198

A Formal Examination of the Literature..................201 
Use Outside of Leadership Development.................202

Directions and Parameters for Use.....................202

Communicating the Technique........................204

Alignment with Appreciative Inquiry.................205

Making Explicit the Theory-in-Use..................207

Conclusion..............................................209

REFERENCES ......................................................... 212

APPENDIX A - MAPPING LEADERSHIP DEVELOPMENT ....................227

APPENDIX B - RESULTS FROM ALPHA COMPANY ..........................229

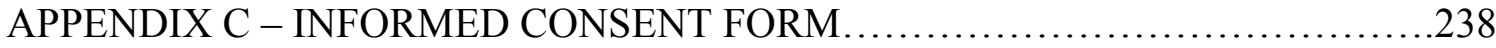




\section{INTRODUCTION: CHAPTER ONE}

Throughout history, scholars have written about the concepts of leadership and leadership development; according to Bass (1990), "leadership is a universal phenomenon in humans" (p. 4). Although its terminology and name has changed over time, leadership development - the process of developing leaders - has been on the minds and in the writings of major scholars. Confucius encouraged leaders of his time to set the moral example and, during four dynasties, Confucian education touched not only the prestigious, but also "commoners." Further, the principles of Taoism promoted the concept of "servant leadership" which places high value on people feeling that they have accomplished a task themselves without the help of a leader. In 2300 B.C., the Egyptians wrote hieroglyphics for leadership, leader, and follower and taught three attributes of a Pharaoh. Later, Plato suggested the notion of the Philosopher King; a man who endured years of education prior to taking his role as "leader." The Greeks discussed concepts and attributes such as justice and judgment, wisdom and counsel, shrewdness and cunning, and valor and activism. In what may have been the first research on leadership, Plutarch compared the leadership styles of 50 Roman and Greek leaders. In The Prince,

Machiavelli (1505) wrote, "There is nothing more difficult to take in hand, more perilous to conduct, or more uncertain in its success, than to take the lead in the introduction of a new order of things" (chapter XIV, para. 1). Later, Napoleon listed what he felt were the 115 qualities of a military leader. However, after thousands of years of thinking about leadership and how to develop leadership capacity, many questions remain. 
Today, the topic of leadership development remains an important one, and the numbers are staggering. For example:

- In the last decade, corporate expenditures for leadership development have surpassed $\$ 45$ billion (Vicere \& Fulmer, 1997). By some estimates, organizations spend more than $\$ 200$ billion annually on training interventions (Salas \& Cannon-Bowers, 2001).

- The American Society for Training \& Development (ASTD) reported that 60 percent of Fortune 500 companies surveyed in 1995 listed leadership development as a high priority - up from only 36 percent in 1990. The ASTD survey also revealed that more than 75 percent of responding firms sponsor leadership development initiatives of some type, and that 79 percent believe that leadership development is gaining in importance in their organizations.

- A survey sponsored by Training (Delahoussaye, 2001) found that 75 percent of organizations with 10,000 or more employees spend in excess of $\$ 7,500$ per employee on leadership development annually. Smaller firms of less than 500 spend about $\$ 6000$ per employee. The same study found that 21 percent of the 639 companies mandate participation in leadership development initiatives.

- About 85 percent of companies use classroom training as the primary vehicle for leadership development education (ASTD, 1995). Formal, in-class leadership development initiatives typically last three to five days and are often delivered at off-site locations. According to Vicere \& Fulmer (1997), "The cost of developing a one-week in-house leadership development program is expensive, ranging from $\$ 75,000$ to $\$ 242,000$ plus delivery costs of an extra $\$ 20,000$ to $\$ 100,000 "$ (p. 267).

\section{Challenges of Leadership Development}

This section focuses on the many challenges inherent in leadership development.

However, I must clarify that my intent for shining a light on the challenges is to make the glass "more full." Negativity and a pure focus on the "gaps" is not my purpose. In fact, it 
is quite the opposite. Appreciative Inquiry and positive psychology have gained popularity in recent years. According to Cooperrider \& Whitney (n.d.) "Appreciative Inquiry is about the coevolutionary search for the best in people, their organizations, and the relevant world around them. In its broadest focus, it involves systematic discovery of what gives 'life' to a living system when it is most alive, most effective, and most constructively capable in economic, ecological, and human terms" (p. 3). I see value in this approach. However, I suggest that it is of benefit to examine the "other side" as well; it is not an either/or; It is an "and."

Ultimately, corporations are spending millions of dollars in an effort to build the leadership capacity of the workforce. Unfortunately, not everyone agrees that it is money well spent. For example, an anonymous executive suggests, "Probably at least half of every training dollar we spend is wasted - we just don't know which half' (Martochhio \& Baldwin, 1997, p. 15). Others who are well known in the field of leadership have concerns as well. For instance, Conger (1992) asserts, "Most would agree that to seriously train individuals in the arts of leadership takes enormous time and resources perhaps more than societies or organizations possess, and certainly more than they are willing to expend" (p. 38-39). Are we putting too much stock in leadership development?

According to leadership scholars, additional challenges face leadership development initiatives. Although not always characterized as challenges, the absence of certain criteria potentially introduces inherent problems in design and implementation. These include:

- linkage to business systems.

- evaluation techniques.

- leadership theory. 
- adult learning and adult development.

A number of authors have discussed the need for organizations to link development to the business systems. McCauley, Moxley, \& VanVelsor (1998) assert that

To be fully effective, a development system must be integrated with the organization's other processes: management planning, performance management, job selection, reward and recognition systems, and even mistake systems. The confluence of these processes determines the relative effectiveness of any one development activity. (p. 228-229)

Avolio (1999) agrees, suggesting that organizational culture and norms of practice can serve as barriers to success. Avolio posits that

Training should not be conceived of as a discrete program, but rather as an organizational intervention supported by other interventions over time. Training must have a clear, central purpose that will affect how people perform their roles, ideally, the best training programs create a sense of identification with the core values and beliefs they are attempting to transfer to participants. (p. 130)

Similarly, Conger \& Benjamin (1999) stress that

Organizations themselves need to accept greater responsibility for post program activities. In practical terms, this means establishing a set of expectations for the participants upon completion of the program as well as a system of tangible rewards. It means providing a method of monitoring participants' progress toward meeting prescribed goals. Currently these types of appraisals rarely occur. (p. 65)

Evaluation of leadership development initiatives is another common discussion point of leadership development scholars. According to Avolio (2005), those interested 
will find that only ten percent of the leadership development interventions evaluate past Kirkpatrick's first level (reaction). Conger (1992) asserts that

The value of leadership is difficult to measure. The answer is that you cannot. This dilemma makes it extremely difficult for companies to commit large sums of money to something from which they will see no immediate tangible results. We want to see what we pay for. Leadership is an elusive, long-term investment, especially for a society that often looks only to the next quarter or the next year. (p. 190)

On the other hand, Avolio (2004) suggests that

Evaluating leadership development programs, is essentially testing the construct validity of the model that underlies leadership development. Taking the full range model as an example, there is an expectation that transformational leadership transforms followers into leaders. Having a valid theoretical model to guide leadership development efforts is fundamental to understanding how this 'black box' works. (p. 93)

Avolio's assertion leads the discussion to leadership theory as the third challenge facing leadership development initiatives (e.g., Avolio, 1999; Avolio, 2004; Avolio, 2005; Cacioppe, 1998; Conger, 1992; Conger \& Benjamin, 1999; Goleman, Boyatzis \& McKee, 2002; Popper \& Lipshitz, 1993; Vicere \& Fulmer, 1998). Each theory of leadership has inherent benefits and drawbacks. Regardless of the theory, leadership development initiatives should rest (as Avolio alluded) upon solid leadership theory. The theory provides the roadmap for what leadership development architects are hoping to develop in others. A leadership development initiative not built on a theoretical 
foundation is at a disadvantage and, in extreme cases, may teach concepts and topics having little to do with leadership.

An additional challenge is a lack of intentionally incorporating adult learning theory. Some authors mention this notion in passing, but rarely expand (e.g., Avolio, 1999; Conger \& Benjamin, 1999; Goleman, et al., 2002; London, 2002; Murphy \& Riggio, 2003; Wright, Rowitz, \& Merkle, 2001). For instance, Goleman, Boyatzis, \& McKee (2002) suggest that leadership development initiatives should be "based on the principles of adult learning and individual change" (p. 234). However, the authors offer few suggestions.

Similarly, a leadership development initiative should incorporate principles of adult development theory. In the phrase leadership development, the word development connotes change. If initiative architects hope to develop leaders, they should realize that they are asking leaders to change. Initiative architects are inviting leaders to: expand their world view; become aware of biases, prejudices and perceptions; potentially to create new insights; to become more self-aware and; change behavior. Heifetz \& Linsky (2002) suggest that "To persuade people to give up the love they know for a love they've never experienced means convincing them to take a leap of faith in themselves and in life" ( $p$. 26). Incorporating adult development theory into the discussion of leadership development helps program architects create better development experiences. In his book Learning to Lead, Jay Conger (1992) sums it up well. He suggests:

The development of leadership ability is a very complex process. It starts before birth, with a prerequisite of certain genes that favor intelligence, physical stamina, and perhaps other qualities. Family members, peers, education, sports, and other childhood experiences then influence the child's need for achievement, power, 
risk taking, and so on. Work experiences and mentors shape the raw leadership materials of childhood and early adulthood into actual leadership by providing essential knowledge and behavioral skills. Opportunity and luck are the final determinants of who gets a chance to lead. (p. 33)

\section{The Theoretical Framework}

Part of the answer to the above mentioned challenges can be found in an organization's employees. Every organization is comprised of individuals who have tacit knowledge. Polyani (1983) describes tacit knowledge when he suggests:

We can know more than we can tell. This fact seems obvious enough; but it is not easy to say exactly what it means. Take an example. We know a person's face, and can recognize it among a thousand, indeed among a million. Yet we usually cannot tell how we recognize a face we know. (p. 4)

I assert that the same is true of leadership development practitioners; they know when things are going well and when problems exist. They have years of experience, and there is opportunity to make this knowledge explicit. Moreover, making this knowledge explicit may be one part of the answer to addressing the previously mentioned challenges. Helping an organization uncover its implicit theory of action may be a starting point when assisting organizations in the creation (and continuation) of leadership development interventions that are transforming in nature. Chris Argyris and Donald Schön (1978) introduced the concept of theory of action. According to Argyris (1997):

Human beings hold two different master designs. The first incorporates the theories humans espouse about dealing effectively with others. The second design involves the theories of action they use (i.e., their theories-in-use). Whenever any 
issue is dealt with that activates embarrassment or threat, we have found a systemic discrepancy between the espoused theories and the theories-in-use and a systemic unawareness of the discrepancy while individuals are producing it. (p. 10)

Michael Quinn Patton took the above concept and developed the user-focused theory of action approach. According to Patton (1997), this process assists program developers in uncovering their theory of action. This involves bringing people together in an effort to "make explicit their assumptions and generate a model that could then be tested as a part of an evaluation" (p. 221); testing why practitioners do what they do and why they think what they do yields a desired result. At times, the theory of action is unknown to the practitioner and assisting in making this known can be the first step in examining assumptions, gaps in logic, and in the spirit of AI, opportunities. According to Patton (1997), a researcher using this method must do at least five things:

1. Make the process of theory articulation understandable.

2. Help participants be comfortable with the process intellectually and emotionally.

3. Provide direction for how to articulate espoused theories that participants believe undergrid their actions.

4. Facilitate a commitment to test espoused theories in the awareness that actual theories-in-use, as they emerge, may be substantially different from espoused theories. (Please note that this is not a goal of this study)

5. Keep the focus on this to make the evaluation useful. (p. 223) (Please note that this is not a goal of this study)

It is widely established that leadership development initiatives pervade institutions of higher education, not-for-profit organizations and corporate America. 
Scholars in a number of fields have discussed the need for inclusion of leadership theory, adult development and learning theory, linkage to organizational context, development tools and a sound methodology for evaluation. However, in practice, I suspect that a number of these suggested components are not included which may diminish the learning experience and undercut the overall effect. Therefore, the central purpose of this research was to examine how academic notions of leadership development compare and contrast with the theory of action that guides corporate leadership development initiatives. A secondary purpose was to analyze the process and potential extensions of the userfocused theory of action approach. Argyris and Schön focused their analysis on the discrepancies that occur between espoused theories and theories-in-use. That is not the intent of the present study. Rather, the intent is to determine an organization's theory of action for their leadership development initiatives and then benchmark this with existing literature. As a result, initiative architects have an opportunity to view their theory of action in its entirety and examine potential areas for investigation via the literature.

\section{Definitions}

\section{Leadership Development}

As with the term leadership, the term leadership development has no agreed upon definition. In this section, I review 15 statements that either define leadership development or describe it. Second, I synthesize the definitions and discuss four emerging themes. I then provide a proposed definition of leadership development and share an explanation of its major components.

Scholars have defined leadership development in the following ways: 
- "The stretch of one's capacity to become aware of and build skills around the dynamic of positive leader-follower outcomes" (Davis, 2001, p. 3).

- "planned and systemic efforts to improve the quality of leadership" (Popper \& Lipshitz, 1993, p. 23).

- "The expansion of one's capacity to be effective in leadership roles and processes, which are those things that enable groups of people to work together in productive and meaningful ways" (McCauley, Moxley \& Van Velsor, 1998, p. 4).

- 'Leader development is the expansion of a person's capacity to be effective in leadership roles and processes" (McCauley \& Van Velsor, 2005, p. 2).

- "Leadership development is the expansion of the organization's capacity to enact the basic leadership needed for collective work: setting direction, creating alignment, and maintaining commitment" (McCauley \& Van Velsor, 2005, p. 18).

- "not a program or a one shot training process. It is a system that takes into account how your organization functions, what it rewards, and what it values" (Sindell \& Hoang, 2001, p. 2).

- "Leadership development is a systemic process that begins with assessment of organizational needs, leadership capabilities, and developmental gaps" (Fleishman in London, M., 2002, p. xiii).

- "It can be viewed as a planned intervention in the life stream, where given a particular model, method, time period, and evaluation strategy, we expect to change the course in people's mental model, behavior, and direction of the life stream" (Avolio, 2005, p. 169).

- "Leadership development is the act of expanding the capacities of individuals, groups and organizations to participate effectively in leadership roles and capacities" (Day, 2004, p. 841).

- According to the US Army, "Leader development is [a] continuous, progressive, and sequential process through which leaders acquire skills, knowledge and behavior necessary to maintain a trained Army in peace-time to deter war" (O’Neil \& Fisher, 2004, p. 102). 
- Avolio (2004) defines development as "changes that occur over time due to both maturational processes and learning" (p. 127-128).

- "leadership development should broaden the horizons of participants so that they can see and understand different realities or alternative courses of action. At its best, leadership development should inspire and enable leaders to higher and higher levels of achievement" (Vicere \& Fulmer, 1996, p. 17).

- "to increase the capacity of the whole system to make sense of direction, commitment and adaptive challenges at all relevant levels of understanding and responsibility...The goal of leadership development would be for everyone, from entry-level operational employees on through first-line supervisors, middle managers, directors, vice- presidents and the top managers to construct a sense of what responsibility for leadership is appropriate and useful, how such a responsibility is carried out within their interrelationships in the organization, and when they should be expected to enlarge their sense of responsibility for leadership" (Drath, 2001, p. 165).

- Leader development is "individual-based knowledge, skills and abilities associated with formal leadership roles" (Day, 2001, p. 584).

- Leadership development focuses on "building and using interpersonal competence...key components of interpersonal competences include social awareness and social skills" (Day, 2001, p. 585).

The above statements contain four major themes. First, leadership development should be a "continuous, progressive, and sequential process" (O’Neil \& Fisher, 2004, p. 102) or "a system that takes into account how your organization functions, what it rewards, and what it values" (Sindell \& Hoang, 2001, p. 2). It should not be a one-time training experience. A second theme is that leadership development should "expand the capacities of individuals, groups and organizations" (Day, 2004, p. 841), "broaden horizons so they [leaders] can see new alternatives" (Vicere \& Fulmer, 1996, p. 17), and "change the course in people's mental model, behavior, and direction of the life stream" 
(Avolio, 2005, p. 169). A third purpose or theme is that it should "increase the capacity of the whole system" (Drath, 2001, p. 165). Along these lines, Avolio (2005) describes leadership development at different levels (individual, dyadic, group and strategic) and suggests that leadership development is always a multi-level endeavor. Finally, leadership development should create "positive leader-follower outcomes" (Davis, 2001, p. 3) and it should "enable groups of people to work together in productive and meaningful ways" (McCauley, Moxley \& Van Velsor, 1998, p. 4).

In an effort to synthesize the above, I propose the following definition of leadership development:

Leadership development is a continuous, systemic process designed to expand the capacities and awareness of individuals, groups, and organizations in an effort to meet shared goals and objectives.

Leadership development includes "planned and systemic efforts to improve the quality of leadership" (Popper \& Lipshitz, 1993, p. 23). Next, leadership development should challenge and expand the thinking of individuals. Bruce Avolio (2005) suggests, "leadership development is fundamentally a shift in perspective" (p. 77). In addition, leadership development should exist at all levels within the organization; Drath (2001) asserts, "The goal of leadership development in an organization could thus be to increase the capacity of the whole system to make sense of direction, commitment and adaptive challenges at all relevant levels of understanding and responsibility” (p. 165). Finally, leadership development should help the individual and the organization "work together in productive and meaningful ways" (McCauley, Moxley \& Van Velsor, 1998, p. 4) and "inspire and enable leaders to higher and higher levels of achievement" (Vicere \& Fulmer, 1996, p. 17). 
Leadership Development Initiative

A Leadership Development Initiative (LDI) is another way of saying "leadership development program" or "leadership development process."

Initiative Architect(s)

Leadership Development Initiative Architect(s) or Initiative Architect(s) are individuals or groups who have direct or indirect responsibility for the design, implementation, marketing, evaluation, delivery or content of a leadership development initiative.

End user

The individual whom the leadership development initiative is intended; they are the individuals participating in the development activities.

\section{Study Design}

Case study methodology served as a container and user-focused theory of action approach served as a technique for data collection in a global, for-profit organization. Sample selection occurred on two levels - the case and the individual sample within the case. Data collection occurred through two primary methods: interview and document review. 


\section{Limitations}

For the purpose of this study:

- Case studies can masquerade as the whole, when they are simply a "part." Moreover, case studies can oversimplify a situation, leading readers to false conclusions about the phenomenon.

- Issues of generalizability are a concern. Case studies are not intended to generalize to a broader population. This case study should not and cannot be generalized to any other organization. However, the case is described in sufficient detail that readers will be able to connect or transfer key findings and insights to their own organizations as appropriate.

- The researcher was the primary instrument of data collection and analysis and limited experience in analyzing data and interviewing could have affected results.

\section{Delimitations - Boundaries}

For the purpose of this study:

- This is a single case and focuses upon a single organization.

- The organization should have the following characteristics:

o Commitment of time - participants agreed to participation and committed to 90 minute meetings at four different times.

o Responsibility - data collection was limited to individuals within the organization with direct decision making authority over the leadership or professional responsibility for the leadership development initiative.

o Organizational experience - participants worked in the organization for at least three years to ensure a foundation of corporate knowledge.

\section{Assumptions}

- The data collected is dependent upon, and assume the honesty and integrity of participants throughout the data collection process.

- Leadership development is an activity with some primacy in the organization. 
- Key informants were willing to participate in the study.

\section{Advance Organizer}

I have discussed the purpose and overall theoretical framework of the study. Future chapters cover the following topics:

Chapter Two - Literature Review

The purpose of Chapter Two is three fold. First, I provide a broad overview of the leadership development literature. Within this overview, I discuss limitations therein. I continue with what I have deemed to be five major aspects of the literature on leadership development: leadership theory, organizational context, adult development and learning theory, development tools, and evaluation. Chapter Two concludes with a summary and rhetorical argument for the necessity of this research.

\section{Chapter Three - Methodology}

Chapter Three begins with a discussion situating myself in the research. In this section, I make transparent the potential political and cultural biases I bring to the study. Case study methodology served as a container and user-focused theory of action approach served as a technique for data collection; both are defined and discussed. Next, I will discuss the pilot study conducted and lessons learned. For the primary study, data collection occurred in a for-profit, global organization, with extensive leadership development initiatives. Interviews with senior leaders and human resource development professionals served as the primary data sources. Documents and program materials 
served as secondary sources of information. Chapter Three concludes with a discussion of methods to address validity, reliability and ethical considerations.

Chapter Four - Data Collection and Results

Chapter Four focuses on the study findings and discusses the specific case examined. I explain the organization's theory of action and the validity assumptions. I then focus on participant reactions and thoughts gathered at a debriefing meeting. The chapter concludes with a comparative benchmark of "Beta Company's" approach with the literature on leadership development.

\section{Chapter Five - Implications}

Chapter Five is divided into three sections. The first section is an examination of potential gaps within the leadership development literature. The second focuses on the user-focused theory of action approach and offers suggestions for practice. The third section focuses on the user-focused theory of action approach and its potential extensions within academic and business settings. 


\section{LITERATURE REVIEW: CHAPTER TWO}

The purpose of this study was to examine how academic notions of leadership development compare and contrast with the theory of action that guides corporate leadership development initiatives. A secondary purpose was to analyze the process and potential extensions of the user-focused theory of action approach. Chapter One introduced the background, purpose and problem statement. The purpose of Chapter Two is three fold. First, I provide a broad overview of the leadership development literature. Within this overview, I discuss limitations therein. I continue with a review of what I have concluded to be five major aspects of the literature on leadership development: leadership theory, organizational context, adult development and learning, development tools, and evaluation. Chapter Two concludes with a summary and rhetorical argument for the necessity of this research.

\section{The Leadership Development Landscape}

The literature on leadership development is a disparate and segmented base of literature. Authors writing on the topic of leadership development hail primarily from two fields: business (e.g., Jay Conger, Albert Vicere \& Robert Fulmer), and psychology (e.g., Bruce Avolio, David Day, Manuel London \& Cynthia McCauley). To a smaller extent, not-for-profit foundations and the military have also made contributions; however, this study does not focus on grass roots or military notions of leadership and leadership development. Information on leadership development in organizational life is located in three primary locations: books, journal articles and through organizations such as 
Linkage, Inc., the Center for Creative Leadership and Lominger. Relatively little of the literature is empirically validated.

The literature covers a number of topics with an emphasis on what I call development tools. Development tools are activities that facilitate learning. The primary focus of these articles surrounds development tools such as 360-degree assessments, coaching, action learning, instruments, developmental relationships, and the like. Less common are publications that cover issues such as evaluation, adult learning, adult development and linkage to organizational context. To date, I have located only one journal article focusing on the wider scope of leadership development which is David Day's (2002) Leadership Development: A Review in Context (which devotes more than half the article to development tools). In addition to Day's article, The Center for Creative Leadership (CCL) authored the most comprehensive overview of the topic in its Handbook of Leadership Development (McCauley \& Van Velsor, 2005). The handbook covers topics such as evaluation, linkage to business systems, diversity, and development tools. It also discusses the authors' philosophy on developing leadership capacity. Other topics found in the literature include definitions of leadership development, models of leadership development, the process of developing a leadership development initiative, adult learning theory and leadership theory extended to practice. To a smaller extent, topics such as leadership development in relation to race, gender, curriculum, technology and trends are included (see Appendix A).

However, I have not found an author who has investigated the concept of helping an organization make explicit its implicit theory of action in an effort to help construct leadership development initiatives based upon sound causal linkages; more specifically, 
how to consciously link initiative objectives with activities, systems, context, curriculum and the like.

A number of authors have proposed a desired process for developing leadership development initiatives (e.g., Cacioppe, 1998; Giber, Carter, \& Goldsmith, 2000; London, 2002; Van Velsor, Moxley \& Bunker, 2005; and Vicere \& Fulmer, 1998). They generally focus on the following components.

1. Business Diagnosis - Also called articulate strategic imperatives, this phase examines the rational and business driver for creating a leadership development initiative. This may also include a gap analysis, organizational diagnosis or scan of the environment.

2. Set Objectives for Development - Once strategic imperatives are determined, architects should set objectives for the leadership development process. These outline how the strategic imperatives turn into behaviors on the job.

3. Program Design - The program design includes dozens of interventions or development opportunities (also known as development tools). These may include fellowships, job enrichment/enlargement, personal development plans, action learning, 360-degree feedback, feedback-intensive experiences, coaching and instruments. Here, Vicere \& Fulmer (1998) and Cacioppe (1998) also recommend that organizations select providers to conduct the programming.

4. Implementation - This phase constitutes the formal leadership development initiative.

5. On-the-Job Support - This phase is concerned with the question, "How will learning in the program transfer to the job?" Vicere \& Fulmer (1998) articulate the need to link the development process to the human resource systems. These HR systems may include hiring, evaluation, career development, succession planning and performance management.

6. Evaluation - The final phase in the leadership development process is evaluation. 
Along with the six step process outlined, the above mentioned authors share examples of organizations that have embraced an aspect of this process. For instance, in their book Linkage, Inc. 's Best Practices in Leadership Development Handbook (2000), the authors share a number of case studies, but focus on only one aspect of leadership development. For instance, AlliedSignal created a “360-degree assessment-based leadership development initiative for leaders at all levels of the organization, designed to work in conjunction with the organization's human resource strategic plan and performance management process" (Giber, Carter, \& Goldsmith, 2000, p. 37). While the authors provide examples of the intended goals they do little to place them in a larger context. For instance, the authors described the Vision Behind the Initiative section for the case in the following manner - "AlliedSignal had to develop people who lead the company and contribute to its growth. This major task required a thoughtful evaluation of the strengths and development needs of current and potential leaders" (p. 39). I am interested to know more about Allied Signal's assumptions about the linkages between the current course of action and their desired output. Helping architects of leadership development initiatives critically examine their theory of action is of great importance. Doing so helps identify gaps in logic and help individuals examine the assumptions upon which their leadership development initiatives are predicated.

\section{Limitations of the Leadership Development Literature}

A thorough review of the literature identifies three significant limitations. First, there is little empirical support for the literature on leadership development (Day \& 
O’Conner, 2004). In general, authors such as Jay Conger, Albert Vicere, Robert Fulmer, Bruce Avolio and David Day base their writing largely upon their experience of working with, or studying organizations. For instance, in his book Leadership Development in Balance, Bruce Avolio (2005) proposed the model located in Figure 1.0.

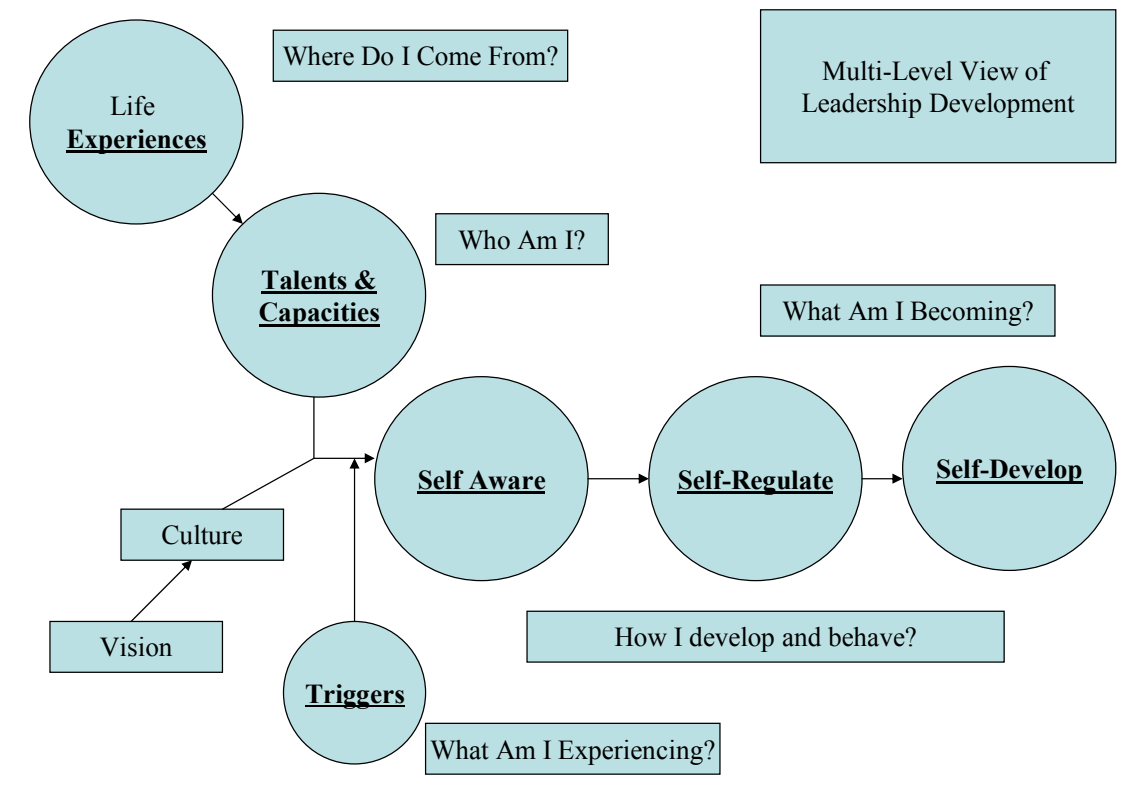

\section{Figure 1.0}

This model may have face validity but, beyond that, little is known about its empirical value. Moreover, authors offer assertions such as "To leverage the impact of leadership development efforts, they must be tightly linked to the organization's human resource management infrastructure, including performance management and reward systems, recruitment and selection procedures, and succession and executive resource planning processes. This final step ensures that a learning orientation becomes ingrained within the organization's culture and operating philosophy" (Vicere \& Fulmer, 1996, p. 
92). The assertion has clear face validity, but I know of no research that supports Vicere \& Fulmer's assertion. The area of leadership development with the strongest base of research is the literature on development tools such as developmental relationships, assessment centers, coaching and action learning. However, this body of empirical research is mixed.

A second flaw is that white, middle class males are the primary authors of literature on leadership development. The Center for Creative Leadership's Handbook of Leadership Development (2005) is the only publication advancing issues about leadership development and gender or leadership development and race. Publications with a clear bias toward white middle-class America bring inherent issues of generalizability when gender, race and the global community enter the conversation.

A third flaw of the literature is a lack of coherence. I have not found a single source for the various models and definitions of leadership development. Individual authors such as Bruce Avolio tend to advance their own model (in his case, the Full Range of Leadership Model) or organizations such as Linkage, Inc. do little more than provide examples of best practices found in organizations with which they work. As previously mentioned, the Center for Creative Leadership's Handbook of Leadership Development comes closest to an "all inclusive" discussion but even it does not cover leadership theory, adult learning theory, technology, a discussion of the various models, or descriptions of development tools such as action learning, e-learning, outdoor education and classroom-based education. 


\section{Five Critical Components}

For the purpose of this dissertation, I intentionally limit the scope of the literature to five components fundamental to leadership development within an organization: leadership theory, organizational context, adult development and learning, development tools, and evaluation. These five components, given proper attention, result in a strong foundation for a leadership development initiative. Each could comprise an entire literature review. As a result, I discuss only the major features of each and direct the reader to additional sources for further investigation.

\section{Leadership theory}

The leadership development process should be rooted in leadership theory (e.g., Avolio, 1999; Avolio, 2005; Cacioppe, 1998; Conger, 1992; Conger \& Benjamin, 1999; Goleman, Boyatzis \& McKee, 2002; Popper \& Lipshitz, 1993; Vicere \& Fulmer, 1998). Returning to Avolio (2004):

Evaluating leadership development programs is essentially testing the construct validity of the model that underlies leadership development. Taking the full range model as an example, there is an expectation that transformational leadership transforms followers into leaders. Having a valid theoretical model to guide leadership development efforts is fundamental to understanding how this 'black box' works. (p. 93)

For example, I worked in a medical center where a solid leader focused on the

following areas: customer, quality, community, culture and finance. All may be important in driving business results, but are they fundamental components of leadership? This is debatable. 
Leadership development should lie on a foundation of theory - not necessarily one specific theory, but theory nonetheless. By doing so, the leadership development initiative has a roadmap that not only provides a description of desired behaviors, competencies and/or skills, but also allows for evaluation down the road. As an aside, no one theory of leadership has all the answers; all have inherent benefits and drawbacks. For example, situational leadership has been panned by a number of authors in the literature (e.g., Blank \& Weitzel, 1990; Fernandez \& Vecchio, 1997; Goodson, McGee, \& Cashman, 1989; Hambleton \& Gumpert, 1982; Vecchio, 1987), but remains popular among practitioners in organizations (Fernandez \& Vecchio, 1997). On the other hand, transformational leadership has a stronger base of research behind it (Coleman, Patterson, Fuller, Hester \& Stringer, 1995; Gasper, 1992; and Lowe, Kroeck, \& Sivasubramaniam, 1996), but has not been embraced by the masses (corporate America). Contingency theory had strong empirical backing as well (Peters, Hartke \& Pohlman, 1985; Strube \& Garcia, 1982) - but where is it today? Research and practice of contingency theory has been at a virtual standstill since the early 1980s.

Leadership theory should be incorporated at some level and, as participants encounter differing contexts, they are better prepared to draw from a number of theories rather than a single approach. Leadership is a relationship between the leader, the followers and the context and as the context and makeup of the followers change, leaders should adjust their approach. Effective leaders are cognizant of, and adjust to, varied contexts. 
The organizational context

The organizational context is a topic discussed by a number of authors who write about leadership development (see Appendix A). In A Systems Approach to Leadership Development, Moxley and O'Conner-Wilson (1998) emphasize the importance of a supportive organizational context and assert that four components of organizational context exist: business context, target population, shared responsibility and supportive business systems.

How the leadership development initiative links to business objectives or context is one component of organizational context (e.g., Cacioppe, 1998; Giber, Carter \& Goldsmith, 2000; Klein, \& Ziegert, 2004; London, 2002; Moxley \& O’Conner-Wilson, 1998; Van Veslor, McCauley \& Moxley, 1998; Vicere \& Fulmer, 1998). The business context includes organizational objectives and how leadership development assists an organization in meeting those objectives. For example, the first phase of the BennisLinkage, Inc. process (Giber, Carter \& Goldsmith, 2000) is business diagnosis. This phase examines the rationale and business driver for creating a leadership development initiative. This may include a gap analysis, organizational diagnosis or scan of the environment. Questions in this phase may include:

- What are the company's current strengths?

- What are the gaps that should be bridged to avoid difficulty?

- How can leadership development help the organization meet its objectives?

Next, London (2002) suggests the need to determine appropriate leadership skills or competencies. A competency is "an underlying characteristic of a person which results in effective and/or superior performance in a job" (Boyatzis, 1982, p. 21). 
Along with organizational context, the organization should determine the target population for training (e.g., Conger \& Benjamin, 1999; Goleman, et al., 2002; Moxley \& O'Conner-Wilson, 1998). Of course, this variable changes with the context; however, it is important to identify how those in the target population assist the organization in meeting its strategic objectives. Of course, some suggest that leadership development should occur at all levels. Returning to Drath (2001):

The goal of leadership development in an organization could thus be to increase the capacity of the whole system to make sense of direction, commitment and adaptive challenges at all relevant levels of understanding and responsibility...The goal of leadership development would be for everyone, from entry-level operational employees on through first-line supervisors, middle managers, directors, vice presidents and the top managers to construct a sense of what responsibility for leadership is appropriate and useful, how such a responsibility is carried out within their interrelationships in the organization, and when they should be expected to enlarge their sense of responsibility for leadership. (p. 165)

Whether or not Drath's vision is feasible depends largely on the organizational context and how it links its strategic imperatives to the leadership development initiative. However, Drath's assertion (2001) is an important one. In his book, Leadership Development in Balance (2005), Avolio suggests that leadership development is always a multi-level endeavor.

Along with the target population, a shared responsibility for development should exist. Leadership development cannot be the sole responsibility of one individual or department to ensure success. In an organization with a culture of leadership 
development, this responsibility weaves through systems and processes and is everyone's responsibility.

Within the leadership development literature, the following supportive business systems are addressed: technology (e.g., Avolio, 2005; O’Neil \& Fisher, 2004; Spreitzer, 2003; Vicere \& Fulmer, 1998), personal development plans (e.g., Giber, Carter \& Goldsmith, 2000; McCauley, 2001), reward systems (e.g., Bass 1990; Klein, \& Ziegert, 2004; McCauley, 2001), the immediate supervisor (e.g., Bass, 1990; Conger \& Benjamin, 1999; Yukl, 2002), hiring (e.g., Conger, 1989), succession planning (e.g., Bass, 1990; Giber, Carter \& Goldsmith, 2000; McCauley, 2001), career development (e.g., London, 2002; Yukl, 2002) and performance management (e.g., Giber, Carter \& Goldsmith, 2000; London, 2002; McCauley, 2001).

In her chapter Leadership Development in the Virtual Workplace (2003), Gretchen Spreitzer discusses a number of technologies that may assist in the process of leadership development. These include desktop video conferencing, collaborative software systems, and internet/intranet systems. Desktop video conferencing can be installed for approximately $\$ 1,000$ per computer (Spreitzer, 2003) and is a way for individuals to have developmental opportunities and mentors in distant locations. A second resource is collaborative software systems, which "allow users to simultaneously work on documents, analyze data, or sketch out ideas on whiteboards - almost like being physically proximate" (Spreitzer, 2003, p. 76). Avolio (2005) suggests using groupware technology to share development plans and establish peer learning groups. This medium allows virtual action learning teams, individuals, trainers and organizational leaders the opportunity to develop in a virtual workplace. A third resource is internet/intranet 
systems. Leadership development activities can align nicely with an organization's intranet (internal web site). The internet/intranet can serve as an interface for tracking personal development plans, online courses and career development opportunities.

Other forms of technology are gaming and simulations. O’Neil \& Fisher (2004) found that computer games had a number of benefits to adult learning. These include promotion of motivation, (e.g., fun), enhancement of thinking skills, facilitation of metacognition, improvement of knowledge and skills, and building of attitude (p. 106). In fact, the U.S. military has used games such as Doom and Quake for training U.S. Marines. Another program that recently entered the market is SimuLearn's Virtual LeaderAccording to SimuLearn's website, "The Virtual Leader is a program designed to meet the challenges of the new economic era; a program that offers e-learning economies while elevating the coaching effectiveness of your staff" (www.simulearn.net). Other organizations such as the Center for Creative Leadership offer "webinars" which serve as virtual seminars with a well known author or a leading thinker in the field. Another interesting application is Avolio's (2005) use for technology in a coaching process; he suggests, "We are connecting virtual coaches together via online groupware systems, so that they can work with each other to come up with the best strategies for developing others" (p. 170). As the boundaries of organizations become "boundaryless," technology plays an important role in helping individuals develop leadership skills and competencies.

Personal development plans are an individualized approach to leadership development. Taylor and Edge (1997) define a personal development plan as "a process through which the individual prepares a training and development plan, and for which the individual takes responsibility" (p. 21). Higson \& Wilson (1995) developed a three part 
model for personal development plans. Part one calls for a gap analysis. Gaps in performance are suggested and recorded on the personal development plan. Next, Chappelow (1998) suggests choosing a theme which may include:

- focusing on an area of need;

- capitalizing on a strength;

- taking an area of need and developing it into a mid-range skill;

- "compensate for a weakness by owning it and adopting strategies to workaround it. Use a strength to tackle a weakness; and

- addressing an area in which he has limited experience" (p. 54-55).

Part two of Higson and Wilson's (1995) model consists of the learning plan. In this step, the employer and employee identify three "learning needs" which are recorded on the development plan. Chappelow (1998) suggests the following questions as a guide to select a development opportunity:

- Does the goal motivate and energize me?

- Will achieving this goal help me be more effective in my current position?

- Will my organization benefit from this goal? (p. 55)

Part three suggests the implementation of a learning log. The employee should complete the learning log on a weekly basis and record learning moments. The supervisor initials the document and monitors progress. Along with the learning log, individuals should determine a number of learning strategies to help participants achieve desired goals. These may include new job assignments, a developmental relationship, classroombased training, an external learning experience or other developmental experiences. 
One challenge surrounding personal development plans is that organizations do not track an individual's progress (McCauley, 2001). As a result, organizations do not have a track record of how individuals develop over time. However, new technology, may make this easier to accomplish.

Moxley \& O'Conner-Wilson (1998) only briefly mention reward and recognition systems. However, the authors do provide an example of how inappropriate reward systems can undermine a leadership development process:

One organization's leadership development program focused on helping people develop the skills needed to effectively operate in a flatter, more team-based environment. Yet, the performance appraisal and compensation system put more emphasis on individual performance. The reward system undermined the goal of developing a team-based work environment. (p. 229)

McCauley (2001) suggests "reward systems send a clear signal about what is valued in the organization" (p. 372). Organizations that espouse a belief in leadership development but in practice only reward individuals for "making goal" are sending a double message.

An additional aspect of a supportive business system is an individual's immediate supervisor. According to Bass (1990), "most important to whether training will modify behavior back on the job is the trainee's immediate supervisor" (p. 854). In support of Bass' assertion, Huczynski and Lewis (1980) found the level of support and overall attitude of a learner's boss have the greatest effect on transfer of skills. Another study by Facteau, Dobbins, Russell, Ladd, \& Kudisch (1995) supported these findings, which concluded that people who feel a high degree of support from their bosses report a higher level of motivation to attend and learn from training opportunities. 
Hiring is an individual's first impression of the organization. It is an opportunity to explain the job requirements, competencies, and expectations for employment from the beginning. Jay Conger (1989) asserts that "companies should begin leadership development at the very moment of recruiting" (p. 162). Organizations that have connected the dots and linked organizational context with leadership development have an opportunity to articulate clearly the corporate culture and development expectations during the hiring process. This allows individuals the opportunity to assess whether or not personal values align with organizational values.

Succession planning/management is a hot topic. However, some research suggests that organizations are not addressing this issue. For instance, one study cited by Wells (2003) found that only one-third of the 428 human resources professionals studied had implemented succession planning. Another study of 200 human resources professionals found that 94 percent of human resources professionals felt that their organizations had not "adequately prepared their younger workers to step into senior leadership positions" (Wells, 2003, p. 46).

Fulmer and Conger (2004) define succession planning as "all about identifying talent - what it looks like, who has it, who needs to develop it and how it can best be developed" (p. 11). However, if organizations are not adequately managing this process, it is difficult to plan for the development of individuals within the organization.

In The Leadership Pipeline, Charan, Drotter and Noel (2001) suggest that employees should move through six distinct "passages." Each passage brings inherent leadership development opportunities.

- Passage One - From managing self to managing others - Here, the individual moves from a line function to one of managing others in a line function. 
- Passage Two - From managing others to managing managers - According to the authors, organizations lack training for individuals at this level. Charan et al. (2001) suggest that "level two managers select and develop the people who will eventually become the company's leaders" (p. 18).

- Passage Three - From managing managers to functional manager - In this passage, individuals begin managing areas outside their expertise and should understand the interconnections among other functions.

- Passage Four - From functional manager to business manager - In passage four, an individual is running an entire function. According to the authors, individuals must make shifts in a number of skill sets. For example, the authors suggest that leaders integrate reflection and analysis into their functions rather than simply accomplishing tasks.

- Passage Five - From business manager to group manager - This passage requires the ability to run a number of businesses within an organization, and again, requires a new set of skills.

- Passage Six - From group manager to enterprise manager - The "C" level leaders think long-term and place greater emphasis on values and the management of external entities.

Charan, et al. (2001) suggest that each passage has a new set of skill requirements (new capabilities required to execute new responsibilities), time applications (new time frames that govern how one works) and work values (what people believe is important and becomes the focus of their effort) (p. 8). Naturally, the new skills may be completely different than those needed in previous capacities and each has a direct effect upon leadership development.

Career development is another traditional human resource function that, if aligned with leadership development, can affect an individual's development within an organization. Kirk, Downey, Duckett \& Woody (2000) define career development as “a 
process for achieving specific employee and organization goals, including providing career information to employees, helping employees identify advancement opportunities, promoting job satisfaction, and improving employee productivity" (p. 205).

Career development interventions should be an organized set of programs that work together as a system (Leobowitz, 1987). In her research, Leobowitz found that the first step in creating a career development program is defining organizational needs and opportunities. Based on these, a number of interventions are available for use.

In Name Your Career Development Intervention, Kirk et al. (2000) cover a number of interventions relating to career development.

- Alternative career paths - allow employees the opportunity to transfer current skills to a new role within the organization. This may be a lateral move or even downshifting.

- Assessment centers - provide employees with an enormous amount of feedback through the use of instruments. These instruments help employees clarify their goals and identify areas of interest and may focus on aptitude, personality and vocational interest (Bowen \& Hall, 1977).

- Career coaching/counseling - allows an employee the opportunity to work with a "coach" who can help with "planning and implementing his career goals in a one-on-one counseling session" (Zheng \& Kleiner, 2001, p.36).

- Career pathing - a process of "outlining an individual career plan, usually within an organization. Career pathing is most often used as a part of management training and development, although individuals may develop their own career track" (Kirk et al., 2000, p. 207).

- Cross-training - an opportunity for employees to learn new skills outside their areas of expertise. For instance, two individuals in a department may learn each other's tasks in an effort to cover for one another. 
- Dual career paths - prepare an employee to move within the organization, but not necessarily in a vertical direction. After all, not everyone wants to be a manager.

- Flextime - allows employees the opportunity to balance work-life needs; they have the opportunity to choose a schedule that works best for them.

- Job enlargement - sometimes referred to as "horizontal job loading," this intervention increases the number of tasks (at the same level) for which an individual is responsible.

- Job enrichment - also called "vertical job loading," this intervention increases an employee's responsibility within the organization. This technique allows individuals opportunities to expand their skill base without leaving their current role.

- Job rotation - allows an individual the opportunity to see a number of different departments within an organization. Medical students are involved in various rotations throughout training.

- Job sharing - allows two individuals to share one role or function within an organization. Job sharing is a way to retain valued employees who no longer wish to work full time.

- Phased retirement - this method gradually decreases the work schedule of employees until full retirement.

- Sabbaticals - these are extended leaves of absence. This intervention is generally associated with teachers. A sabbatical offers an individual a respite and serves as a retention tool for valued employees.

- Temporary assignments - also known as "project work," this intervention keeps valued employees engaged and can help them avoid burnout. It allows employees the opportunity to gain new skills and even work with new individuals. 
Organizations that integrate career development functions with leadership development maximize their effect and allow flexibility to meet the individual needs of employees.

Along with career development, Performance Management can link to leadership development and not solely measurable business objectives such as widgets sold, budget, and the like. If an organization hopes to develop leaders, an evaluation of leadership abilities makes sense. Linking development to performance appraisal is important for two primary reasons: accountability and culture. First, linking performance appraisal to leadership development adds accountability to the process. To view it another way, tracking the performance of development plans reward those who have truly worked to develop their skills. Linking personal development plans with performance appraisal helps integrate systems and create a culture of leadership development within the organization. If every individual focuses on three concrete and objective goals each year, like compounding interest, capacity grows over time. A culture of leadership development exists when it weaves throughout the organization and serves as a foundation for everything else.

There is an opportunity to weave each of these systems together in a way that will have a greater effect on the individual learner or "end user." Linking these systems together provides a "united front." It provides a culture of development and the benefits could significantly affect an organization's bottom line. Vicere \& Fulmer (1998) suggest that "organizations should make leadership development part of a consistent human resource strategy that blends the processes of recruitment, selection, development, appraisal, and reward into an integrated system for talent pool management, rooted in the 
ideas of the organization and focused on the marketplace" (p. 52). Linking leadership development activities to organizational context builds a foundation for the process, and provides incentives for employees who prioritize learning.

\section{Adult development and learning theory}

How adults develop and learn is of fundamental importance to leadership development. In the phrase leadership development, the word development connotes change. Initiative architects are asking leaders to expand their world view, become aware of biases, prejudices, perceptions and are potentially asking leaders to create new insights. Returning to Heifetz \& Linsky, (2002) "To persuade people to give up the love they know for a love they've never experienced means convincing them to take a leap of faith in themselves and in life" (p. 26). It means that architects of leadership development initiatives view participants as individuals who enter the process at different points based upon their psychological attributes and previous life experiences.

Two pioneers in the field of adult development theory are Erik Erikson and Daniel Levinson. Erikson's theory of identity development permeates the majority of adult development theory. Erikson (1959) suggests that adult development occurs throughout an individual's life span and is not bound by time. He asserts that adults continually face life's dilemmas. The "three stages of adulthood" are intimacy vs. isolation, generativity vs. self-abortion and stagnation, and ego integrity vs. despair. Intimacy vs. isolation focuses on the adult's ability to establish deep and meaningful relationships with other human beings. The second stage of adulthood is generativity vs. self-abortion and stagnation. Generativity is the "interest in establishing and guiding the next generation, although there are people who, from misfortune or because of special 
and genuine gifts in other forms of altruistic concern and of creativity, which may absorb their kind of parental responsibility" (Erikson, 1959, p. 103). Ego integrity vs. despair is the acceptance of one's life cycle and all that has comprised the journey; it is individuals taking responsibility for their destiny.

Like Erikson, Daniel Levinson focused on adult development and authored The Seasons of a Man's Life. Levinson's thinking was similar in that he suggested that all adults move through stages (or seasons). Levinson proposed ten stages.

- Early adult transition

- Entering the adult world

- Age 30 transition

- Culmination of early adulthood: settling down

- Midlife transition

- Entering middle adulthood

- Age 50 transition

- Culmination of middle adulthood

- Late-adult transition

- Late adulthood
1-22

22-28

28-33

33-40

40-45

45-50

50-55

55-60

60-65

$65+$

Like Erickson, Levinson asserted that generally speaking, all adults pass through stages and, although variations exist, he proposes that there is an underlying order in the life course. However, Erikson suggested that adults who pass through these stages have a more healthy and happy adulthood. Levinson differed from Erikson in that he simply viewed the stages (or seasons) as common difficulties associated with a certain age. Levinson (1978) asserts that "the tasks of one period are not better or more advanced than those of another, except in the general sense that each period builds upon the work of earlier ones and represents a later phase in the cycle" (p. 320). 
Robert Kegan's constructivist/developmental theory took the thinking of Erikson and Levinson to another level. Erickson and Levinson see development as a phenomenon rooted in time and stages of life. Kegan, a stage theorist, asserts that individuals may never develop past certain ways of being. Rather than time, the individual is the agent of development and programs that aid in this process are worthwhile.

Constructivist/developmental theory gives attention to how "individuals perceive or make meaning of the world around them" (Avolio \& Gibbons, 1989, p. 286). Kegan \& Lahey (1984) suggest that development is the ability to make meaning of experiences regardless of age. How individuals interpret a situation or an event is dependent upon their life construct and developmental level; this is a subjective process. According to Kuhnert \& Lewis (1987), constructivist personality theories posit that people differ in how they construct and make meaning of experiences in their physical, social and personal environments. The authors suggest that "understanding the process through which people construct meaning out of their experiences may advance our knowledge of how leaders understand, experience, and approach the enterprise of leading” (p. 650).

According to Day (2004), "Individuals at higher levels of development are able to use a greater number of knowledge principles to construct their experiences (differentiation) and to make more interconnections among these principles (integration). This results in a broader perspective on how things are interrelated (inclusiveness)" (p. 43). Therefore, an individual's ways of knowing guide his lives and actions. According to Kegan \& Lahey (1984) this does not link to age, because three different adults could experience the same event and interpret the happenings in three different ways. Kegan \& Lahey (1984), define development as "a process of outgrowing one system of meaning by 
integrating it (as a subsystem) into a new system of meaning; what was "the whole" becomes "part" of a new whole. Kegan (1994) calls this the "subject-object" relationship. According to Kegan,

'object' refers to those elements of our knowing or organizing that we can reflect on, handle, look at, be responsible for, relate to each other, take control of, internalize, assimilate and otherwise operate upon. All of these expressions suggest that the element of knowing is not the whole of us; it is distinct enough from us that we can do something with it.

'subject' refers to those elements of our knowing that we are identified with, tied to, fused with, or embedded in. We have object we are subject. We cannot be responsible, in control of, or reflect upon that which is subject. Subject is immediate; object is mediate. (p. 32)

For example, leaders who have little awareness of their emotions and how they affect others are subject to these behaviors; they do not have control or in some cases, the ability to reflect upon their actions. Kuhnert \& Lewis (2001) describe it this way: "What is subject for some is object for those at higher stages of development” (p. 651). Kegan and his colleagues developed the "subject-object" interview to help determine an individual's epistemology (Lahey, Souvaine, Kegan, Goodman, \& Felix, 1988, n.p.). As a result, according to Kegan, individuals make different meanings of leadership depending on their level of development. Kegan's theory outlines five distinct stages of development but, within the context of this discussion, I examine three: imperial (stage two), interpersonal (stage three) and institutional (stage four).

The imperial stage (stage two) finds individuals focused heavily on individual needs and goals. An example offered by Kegan (1982) is that if individuals at this stage 
do something wrong, they are likely filled with worries of "being caught" rather than guilt. Kuhnert \& Lewis (2001) posit that leaders at this stage only have the capacity to work out of the transactional leadership style (transactional leaders focus on task completion and compliance - these leaders rely heavily on organizational rewards and punishments to influence employee performance). The authors go on to suggest that "Stage two leaders may say that they aspire to higher order transactions (e.g., team spirit, mutual respect), but from the perspective of cognitive/developmental theory they have not developed the organizing processes (subject) necessary for understanding or participating in mutual experiences and shared perceptions" (p. 652). Leaders at this stage do not have the capacity to reflect on their agendas. They are their agendas.

At the interpersonal stage (stage three), leaders focus on personal needs and the needs of others. They can hold their own interests and the interests of others simultaneously. They are more likely to connect with those around them and experience increased levels of trust, connectedness and commitment to others. According to Kuhnert \& Lewis (2001), “whereas the stage two leaders negotiate with their employers to satisfy personal agendas, stage three leaders sacrifice their personal goals in order to maintain connections with their employers. Thus, the key transactions for the stage three leaders are mutual support, expectations, obligation and rewards" (p. 652). Although still working out of transactional leadership style, stage three leaders are moving away from their own needs to an interconnection between their needs and the needs of others.

Stage four is the institutional stage. Kegan (1982) suggests that individuals at this stage have developed a consistency across arenas, developing their own identity. This self-identity and reliance on personal standards and commitments is the hallmark of stage 
four. Stage four leaders, in a sense, "stand on their own." As Kegan (1982) puts it, they move from "I am my relationships" to "I have relationships" (p. 100). They work through what Burns (1978) may call “end values.” At this stage of development, leaders may make their decisions out of a strong set of values and principles rather than goals or relationships. Moreover, the individual has the capacity to reflect and modify these values (Kegan \& Lahey, 1984). According to Kuhnert \& Lewis (1987), "unless leaders have progressed to stage four personality structures, they will be unable to transcend the personal needs and commitments of others and they will be unable to pursue their own end values" (p. 653). Because of this, Kuhnert \& Lewis assert that transformational leadership begins at this level. Although pieces exist in stage three, it is here where an individual acts holistically out of a place of transformational leadership. Kuhnert \& Lewis (1987) assert that “transforming leadership is made possible when leaders' end values (internal standards) are adopted by followers, thereby producing changes in the attitudes, beliefs and goals of followers" (p. 653).

The constructive/developmental view of leadership has a number of implications for the study of leadership and leadership development. First, Day (2004) suggests that individuals at lower levels of development will likely construct leadership out of a place of dominance: a transactional place. According to Day (2004), "this is not a wrong way to construct leadership, but it is inherently limiting because an individual leader is expected to act as a sort of hero" (p. 44). A more sophisticated level of leadership requires interpersonal influence, which may be more inclusive and allow the leader more flexibility. Helping leaders understand and examine where they work from develops selfawareness and provides additional tools for success. 
Second, according Avolio \& Gibbons (1989), “A leader who operates at a lower developmental level than his or her followers cannot transform followers to a higher level than his or her own. Conversely, a leader who views the world from a developmental level that is not understood by his or her followers will also have difficulty transforming followers to his or her way of thinking" (p. 294). The leader may need to be aware of how followers make meaning and approach the conversation or relationship from their level. This is an important piece of the puzzle, because leadership development initiatives should meet people where they are; one size simply cannot fit all. A program developed and constructed at stage four may sound and be completely foreign to an individual at stage two. The concepts of stage four may be a jump. Day and Halpin (2003) agree and suggest "there is an inherent asymmetry in the development process in which those at higher levels of complexity can understand the thinking of those at lower levels (if motivated to do so), but those at lower levels cannot understand the thinking of those at higher development levels" (p. 14).

A third implication for leadership development is the concept of meaning-making and perception. VanVelsor and Drath (2005) exemplify this notion through the following suggestion: "what he learns will be framed and limited by the ways in which he can make what he learned meaningful. Everything learned will cohere within that developmental framework" (p. 396). Each person views the world through a different lens depending on life experience and developmental level. This concept alone can help leaders make better sense of their situation and the environmental context. For instance, leaders who work out of stage three may begin to understand why some have a difficult time understanding them literally and conceptually. If surrounded by a number of competitive stage two team 
members, it will be a challenging task to work together and truly develop a sense of team; team members will be too busy thinking about their own needs.

Finally, Kegan's thinking can increase the self-awareness of the leader. Learning about this and other theories allow leaders an opportunity to reflect on their own developmental stages and how this affects them and their associates. Leadership development initiatives that intentionally assist participants in perspective transformation likely have a greater effect on participants.

Along with adult development theory, adult learning theory is an important factor in the leadership development equation. However, it receives only a cursory mention by leadership scholars. Like adult development, adult learning is a personal process. Merriam \& Caffarella (1999) assert that "the context of adult life and the societal context shape what an adult needs and wants to learn and, to a somewhat lesser extent, when and where learning takes place” (p. 1). For instance, Antioch's Ph.D. in Leadership and Change has had a major effect on the societal context of what it means to earn a Ph.D. Moreover, it has helped adult learners work through the issues of "when" and "where."

Merriam \& Caffarella (1999) suggest five primary orientations to learning: behaviorism, cognitivism, humanist, social learning and constructivist. Behaviorism's primary purpose is to elicit behavioral change in a new and desired direction. While behaviorists are concerned with behavioral change, cognitivists focus on developing “capacity and skills to learn better" (p. 264). Humanists, on the other hand, are primarily concerned with the learner attaining self-actualization and an autonomous, self-directed process to fulfill personal needs. Proponents of social learning examine the intersection 
of the social context and the learner. Finally, constructivists are concerned with the learners' construction of reality and how individuals make meaning from experiences.

In this dissertation, I focus on three of the above concepts: behaviorism, social learning and constructivist (transformative learning). I provide a brief description of each and link their applications to leadership development. I also discuss "transfer of learning" - an important element of any leadership development initiative.

Behavioral learning theorists implement objectives-centered instruction when creating leadership development initiatives. Leadership theories that focus a large portion of time on leader competencies and/or skill building (such as emotional intelligence) benefit from this approach. Behaviorists suggest that reinforcement of learning be quick and undesirable performance corrected immediately. In addition, repetition and testing should occur on a regular basis. As a result, leadership development initiatives should incorporate a number of "real-time" opportunities for learners to practice and perform new behaviors. This real-time practice combined with coaching from independent observers may prove beneficial. Moreover, behaviorists argue that learners be placed in situations that elicit anxiety so an incentive to learn exists. Activities that force participants to move from their comfort zones are valued.

Behaviorists assert that learning occurs when someone wants something and sees learning as a means to an end. Behaviorists suggest that training be linked with a prestige or a desired outcome; a promotion, a degree, a certificate or another reward that motivates learners to incorporate and internalize new behaviors.

Instructors hoping to utilize this method of teaching should: encourage repetition of acts performed correctly, give frequent examinations to gather feedback on the 
learning process, suggest objectives clearly in advance, provide different variations of the same stimuli (because each stimulus-response bond is unique), vary subjects so learners do not become fatigued, avoid punishment, make learning experiences as individualized as possible, measure behavioral change, create an environment of anxiety and allow learners to reward themselves for their accomplishments.

The concepts of behaviorism have real links to leadership development. First, a program design with objectives-centered outcomes likely appease those funding leadership development initiatives. After all, a part of developing leaders rests upon the ability of the leadership development initiative to foster new behaviors that have a positive effect on one's abilities. A second benefit of this approach is the notion that participants see a concrete benefit for participation. A third potential benefit of behaviorism is the notion of stretching learners outside their comfort zones. After all, one goal of all leadership development initiatives is to challenge individuals to practice new ways of being; this can be uncomfortable and challenging for the learner. A number of scholars discuss the concept of challenge as an essential piece of development (e.g., Heifetz \& Linsky, 2003; McCauley \& Van Velsor, 2005).

While behaviorism focuses on repetition, skill, and competency building, social learning focuses on one's environment as major force for learning. Albert Bandura (1977) is the founder of social learning theory which posits that people learn behavior (e.g., leadership, aggression) based on modeling in their environments. Bandura (1977) suggests:

Learning would be exceedingly laborious, not to mention hazardous, if people had to rely solely on the effects of their own actions to inform them what to do. Fortunately, most human behavior is learned observationally through modeling: 
from observing others one forms an idea of how new behaviors are performed, and on later occasions this coded information serves as a guide for action. (p. 22)

Therefore, learning is a relationship between the learner and the environment. Merriam \& Caffarella (1999) suggest, "behavior is a function of the interaction between the person with the environment. This is a reciprocal concept in that people influence their environment, which in turn influences the way they behave" (p. 260).

Interestingly, Charbonneau, Barling, \& Kelloway (2000) explain leadership development through a social learning framework. The authors found that adolescents tend to mirror behavior displayed by their fathers and in turn, display these characteristics with their peers. Additionally, Zacharatos, Barling \& Kelloway (2000) found that, if attributes of transformational leadership exist in youth, this may have a major effect on later leadership in adulthood. In their research, the authors determined that children who perceived their parents to be transformational tended to display these behaviors. These same adolescents were more likely thought of as transformational by their peers and coaches.

Social learning theory is an important piece of the leadership development puzzle. First, leadership is contextual; what works in one situation may not work in another. Leadership development opportunities should help participants better understand their environment and how it affects those within in it. People are products of their environment and have learned what is, and is not, socially acceptable within their organization. At times, the real culture is different from the espoused culture. A culture that promotes communication, honesty, ethical behavior, and transparency may not accept individuals with differing values (and vice versa). 
In addition, social learning underscores the importance of congruence between leadership development and the corresponding culture. Returning to the example offered by Moxley \& O'Conner-Wilson (1998) underscores this assertion:

One organization's leadership development program focused on helping people develop the skills needed to effectively operate in a flatter, more team-based environment. Yet, the performance appraisal and compensation system put more emphasis on individual performance. The reward system undermined the goal of developing a team-based work environment. (p. 229)

Leadership development initiatives that do not align with the "real" organizational culture encounter challenges from the outset. Mixed messages likely occur and, in the end, the individual is forced (or encouraged) to act in a manner congruent with the organization's theory-in-use rather than the espoused theory.

On a more individualized level, social learning emphasizes the need for leaders or teachers to exemplify the desired behavior(s). Proponents of social learning assert that teachers or leaders who do not model the desired behavior undermines efforts to effect lasting change. For instance, supervisors who promote one course of action, yet do not themselves exemplify this behavior likely undermines their efforts. To summarize, people learn behavior(s) based on modeling in their environment; this concept can either help or hinder leadership development initiatives depending on the cultural context once participants return to their work environments.

While behavioral approaches of adult learning focus on skill and competency building, and social learning theory focuses on one's environment, developmentalism closely examines the learner's meaning-making system. Linked closely to the concepts of Kegan's constructivist/developmental theory, perhaps the best known theory of 
developmentalism is Mezirow's transformative learning (also known as transformational learning).

Transformative learning occurs when individuals critically reflect upon their environment and learning. Through intense reflection, individuals transform their thinking and view of the world. Jack Mezirow introduced the topic of transformative learning in 1978. Central to the theory of transformative learning is the notion that adults make new meaning of their experiences. In the words of Mezirow (2000):

That is why it is so important that adult learning emphasize contextual understanding, critical reflection on assumptions, and validating meaning by assessing reasons. The justification for much of what we know and believe, our values and our feelings, depends on the context - biographical, historical, cultural - in which they are embedded. We make meaning with different dimensions of awareness and understanding; in adulthood we may more clearly understand our experiences when we know under what conditions an expressed idea is true or justified. (p. 4-5)

For Mezirow, adult learning is about developing autonomous thinking. According to Mezirow (2000), learning occurs in the following ways: by elaborating existing frames of reference, by learning new frames of reference, by transforming points of view, or by transforming habits of mind. Learning occurs when meaning structures (also known as a "frame of reference") change. Frames of reference are displayed in two distinct ways. One is a habit of mind and the other is a point of view. A habit of mind may be a political stance such as liberal or conservative, a preference for introversion or extroversion and other orientations or world views. A point of view is the habit of mind expressed and “arbitrarily determines what we see and how we see it - cause-effect relationships, 
scenarios of sequences of events, what others will be like and our idealized self image" (Mezirow, 2000, p. 18).

Imel asserts (1998), "perspective transformation explains how the meaning structures that adults have acquired over a lifetime become transformed" (n.p.). Mezirow and others reinforce the need for critical reflection in order for transformative learning to occur. Critical reflection assists learners in confronting their political, economic, social, cultural, and religious viewpoints; allowing individuals to become more aware of how these (and others) affect their view of the world. Regarding critical reflection, Brookfield (1996) asserts, "education is centrally concerned with the development of a critically aware frame of mind, not with the uncritical assimilation of previously defined skills or bodies of knowledge" (p. 17). For example, encouraging adults to better understand the reasoning behind policies, procedures, and cultural norms assists in helping the organization grow and troubleshoot problems or areas of concern.

Another central theme of Mezirow's work is the concept of a disorienting dilemma. A disorienting dilemma is a life event or crisis that forces individuals to see their world, their relationships, and/or their lives in different and new ways. As an aside, it does not necessarily have to be one event; a disorienting dilemma can be a string of events or combination of events that cause people to change their views. Transformative learning fosters a critical change in an individual's meaning structures and, as a result, individuals develop new frames of reference. In ways, transformative learning is the how to Kegan's constructivist/developmental theory of development. As individuals' frames of reference and meaning-making develop, so do their views and perspectives of the world. As a result, this method may help participants increase their self-awareness, which 
is a major theme in the leadership development literature. For instance, Goleman et al. (2002) assert that self-awareness means having a deep understanding of one's emotions, as well as one's strengths and limitations and one's values and motives" (p. 40). Personal growth and self-awareness permeate the literature on leadership development. Personal growth programs are "based, generally, on the assumption that leaders are individuals who are deeply in touch with their personal dreams and talents and who will act to fulfill them (Conger, 1992, p. 45-46).

Another important concept from the adult learning literature is Transfer of learning. Transfer of learning is a crucial piece of leadership development often left unplanned. Caffarella (2002) defines transfer of learning as "the effective application by program participants of what they learned as a result of attending an education or training program" (p. 204). On balance, if the education does not result in perspective transformation, learning, or change in behavior, it could be argued that the investment was a poor one. According to Phillips, Jones, and Schmidt (2000), learning does not transfer to the job in 90 percent of cases. If true, this is a staggering number for those involved in leadership development. Caffarella (2002, p. 212) devotes an entire chapter to this topic and highlights a number of enhancers and barriers to transfer of learning. She also compares these barriers and enhancers at a number of levels. These levels include:

- Program Participants

- Program Design and Execution

- Program Content

- Changes Required to Apply Learning

- Organizational Context

- Community or Societal Forces 
A sample of "Program Design and Execution" (one of the above-mentioned levels) is found in Figure 2.0:

\begin{tabular}{|l|l|l|}
\hline \multicolumn{1}{|c|}{ Barriers } & \multicolumn{1}{|c|}{ Factors } & \multicolumn{1}{c|}{ Enhancers } \\
\hline & Program Design \& Execution & \\
\hline $\begin{array}{l}\text { Instructional methods invoke } \\
\text { passive learning }\end{array}$ & & $\begin{array}{l}\text { Active Learning, including } \\
\text { application exercises, is used } \\
\text { extensively }\end{array}$ \\
\hline $\begin{array}{l}\text { Little match between the training } \\
\text { environment and the applications } \\
\text { context }\end{array}$ & & $\begin{array}{l}\text { Close match between the training } \\
\text { environment and the applications } \\
\text { context }\end{array}$ \\
\hline $\begin{array}{l}\text { Unrealistic transfer-of-learning } \\
\text { strategies or no strategies are } \\
\text { included }\end{array}$ & & $\begin{array}{l}\text { Trasnfer-of-learning strategies are } \\
\text { useful and negotiable }\end{array}$ \\
\hline
\end{tabular}

FIGURE 2.0

Planning for transfer of learning at all levels of programming is a crucial step in the learning and leadership development process. Taking the above model as an example, I recently taught an introduction to business course for undergraduates. When discussing a matrix organizational structure or human resources functions, a natural barrier is that there is little match between the learning environment and the application context. In fact, a student may not have an opportunity to experience this context for years. Therefore, it is a foreign concept and will likely be lost by the time the undergraduate experiences it first hand. On the other hand, if I were working with adult students who work in a matrix organization and interacted with human resources on a regular basis, a different learning experience would exist. 
Another approach to transfer of learning is offering post-program activities. The Center for Creative Leadership incorporates these in their feedback-intensive programs (FIP). According to Guthrie and King (2005), "we know that change takes time. Research indicates that it can take more than a year to begin to perceive a behavior change as a result of a single FIP experience (p. 32). However, initiative architects have instituted the following to aid in learning transfer:

- Individual activities

o Goal letters

o Goal setting reports

o Formal coaching

o Blended application

- Informal coaching

o Peer-group discussions

o Learning partners

o Alumni programs

- Organizational activities

o Action learning

o Program debriefings

o Extended use of 360 instruments

To conclude this section, I return to Heifetz \& Linsky (2002) who suggest, "Habits, values, and attitudes, even dysfunctional ones, are part of one's identity. To change the way people see and do things is to challenge how they define themselves" ( $p$. 27). Every individual is a product of nature and nurture. Adult development and learning theory helps individuals examine what has shaped them. For some, family has had an enormous effect. For others, it may be athletics, work, their circle of friends, and/or a simple accumulation of life events. Understanding and studying this concept can be 
beneficial to leaders for a number of reasons. First, it assists leaders in "getting their own shops in order." As Bass (1985) suggests, leaders with "their own shops in order" are in a better position to work out of the "end values." Second, it helps leaders better understand those around them. Leaders who are aware of these adult development and learning dynamics are in a better position to assist others in their development. Finally, the combination of understanding self and others help leaders better understand the context. In some instances, a stage two (transactional) style of leadership may be appropriate. In others, it will not. Adult development theory can open the door for the discussion of context and its relevance to leadership development. However, white males have done the vast majority of writing on the topic. Of course, this fact brings with it inherent challenges; primarily that it views development from a lens of privilege.

\section{Development tools}

Another theme in the leadership development literature is the use of learning activities to accommodate different learning styles and objectives. For this dissertation, I call these development tools. Development tools take on differing characteristics and are the primary methods for delivering leadership development learning activities before, during and after the leadership development initiative. At times, development tools are mixed and matched depending upon the objectives of the initiative. At times, organizations use single development tools as the mechanism for leadership development. In reality, a combination of development tools likely yield the best results (McCauley, et al., 1998). Examples of development tools include: job rotation, job enlargement, job enrichment, developmental assignments, games, simulations, e-learning, 
360-degree feedback, assessment centers, instruments, feedback-intensive programs, equine leadership development, fellowships, service learning, sabbaticals, hardships, personal development plans, action learning, coaching, outdoor education, classroombased education and developmental relationships.

All development tools have benefits and drawbacks (depending upon the context) and each has its time and place in a leadership development initiative. This section examines the following development tools: developmental relationships, developmental assignments, and feedback mechanisms. For each, I provide a brief and general description and provide examples. The primary purpose of the section is to provide a broad brush. By no means is it an all-inclusive description of each development tool. However, it is important to have a general idea of how development tools aid in the leadership development process. Please note that, by far, the most popular development tool is classroom-based training. For a detailed description of this topic, see Salas \& Cannon-Bowers (2001) and Tannenbaum \& Yukl (1992).

Relationships exist at all levels within an organization. Depending on when and where individuals entered the organization, it is likely that a network of relationships has developed. These relationships, formal and informal, can be important to the leadership development process. Developmental relationships are a "means for providing an individual with the information, support, and challenge which they need now to meet their development needs" (Clarkson \& Shaw, 1992, p. 24).

Developmental relationships may be formal or informal in nature. For instance, a formal developmental relationship may occur in a mentor/protégé relationship. An informal developmental relationship may simply be a senior manager "who facilitates the 
career advancement and personal growth of a less experienced manager" (McCauley \& Young, 1993, p. 219). McCauley and Douglas (1998) suggest that employees have different needs depending upon their situation and career level and, as a result, may benefit from a number of developmental relationships. The authors offer five suggestions for helping individuals develop a plan for creating developmental relationships:

1. Seek out multiple relationships for development - People should cultivate a number of relationships at varied levels of the organization. In addition, relationships outside the organization may prove beneficial depending upon the need of the individual.

2. Figure out which roles are needed for the current development goals and find the right people for those roles - It is likely that individuals have a number of areas for development. Awareness of these areas and a rich network of people who can assist, benefit the individual and aid growth.

3. Fully use lateral, subordinate, or external relationships - Relationships should not be limited to individuals above the individual seeking development. This is limiting. Developmental relationships are found in peers, subordinates and outside the organization.

4. Don't assume that relationships need to be long-term or intense to be developmental - With a clear purpose for the relationship in mind, individuals have an opportunity to determine if objectives area accomplished. Not all relationships are life-altering or lifelong in nature.

5. Be especially aware during times of transition - McCauley \& Douglas (1998) suggest that individuals should be particularly aware of their developmental needs during times of transition. Developmental relationships can assist in networking or merely serve as a sounding board.

McCauley \& Young (1993) assert that

if organizations are to enhance management development through more access to relationships, and if they are to do it in a way that moves beyond existing in-house 
assigned mentoring programs, they should understand (a) what makes an experience developmental; (b) which roles of the myriad others can play in our experiences, are central to development; and (c) how various strategies compare in terms of the developmental roles provided. (p. 220)

McCauley and Young (1993) also discuss four types of developmental relationships: Peer networks, work-site change partners, executive facilitators and process advisors. These relationships were used in specific programming offered, but natural bridges could be made to organizational practice. Peer networks are developmental relationships that pair individuals at the same level within the organization. At times, these relationships are based on knowledge. For instance, someone may have certain skills or areas of expertise that the other individual can learn. Work-site change partners are individuals within the organization with whom individuals can pair to help facilitate changes in their work lives. For instance, two individuals may agree to hold one another accountable for meeting a goal. Executive facilitators are individuals with whom executives can confide and learn. Also known as executive coaches, these individuals may provide feedback and serve as "general counsel" to the executive. Process advisors are process experts on a given topic. These individuals "maximize self-awareness building by helping participants interpret and integrate their scores on various instruments and the feedback they receive during the program" ( $\mathrm{p}$. 226).

The literature is supportive of developmental relationships even though determining return on investment for this method can be difficult. Through developmental relationships, leadership development architects have an opportunity to 
help employees develop networks that can assist in a number of ways. On an individual level, the employee may feel more connected and will have an outlet for questions, feedback and development. On an organizational level, developmental relationships can create a web of relationships at multiple levels of the organization. This could aid in retention, recruitment, development and overall employee satisfaction. However, organizations and individuals ought to be intentional about establishing these relationships; without this intentionality, potential opportunities for development are lost. Developmental assignments are changes in size or scope that effect the learning and growth of an individual. Under the umbrella of developmental assignments, I discuss the general concept along with specific methods such as job rotation, job enrichment and action learning.

Along with classroom-based training, developmental assignments (also known as job assignments) are perhaps the most widely-used development tool in corporate America. Historically, this is the primary development tool for organizations. Conger (1989) asserts that "research shows that challenging assignments are the most helpful experience for developing executive talent" (p. 166). In their book The Lessons of Experience, McCall, Lombardo, and Morrison (1988) studied 91 successful executives to determine which development tools helped them along the way. The vast majority reported that experience was the most effective. According to the research, most modern day CEOs did not have coaches and classroom training; real life business was their classroom.

McCall, et al. (1988) found that executives "learn when they need to or have to... because of the demanding nature of these assignments, learning was not a nicety - 
something to be done out of interest or because it might be helpful. Learning was something these managers did because they had no choice but to take action - stab at problems even if they weren't sure what they were doing, because doing nothing was surely unacceptable” (p. 63). In Kotter's (1982) research on general managers, he found that, throughout their careers, the GMs he studied changed positions every 2.7 years. This may support the notion held by McCall et al. (1988) that these individuals did not spend time in the classroom; they were too busy learning in "real time."

For assignments to be developmental in nature, two attributes should be present. First, there should be a level of challenge in the assignment for it to be "developmental" (Brutus, Ruderman, Ohlott, \& McCauley, 2000). Second, developmental assignments should provide individuals with the opportunity and motivation to learn. The opportunity to try out new skills, behaviors and thinking is crucial (McCauley, Ruderman, Ohlott, \& Morrow, 1994).

Because work and life offers a number of opportunities for development, a number of experiences cited by executives as developmental exist. In their research, McCall, et al. (1988) found that these include: early work experiences, first supervisory job, project and task force jobs, line-to-staff switches, starting from scratch, fix it/turn around jobs, and a leap in scope. Early work experiences are an individual's introduction to corporate life. In early work experiences, formal education meets "real life." It is here that individuals realize that classroom learning does not always come to fruition in the “real world." An individual's first supervisory position is another learning moment recognized in the research of McCall, et al. (1988); a whole host of new learning moments await the young manager. Developmental projects or task forces are another 
type of developmental assignment. These are specific opportunities to serve on a committee and work through a particular organizational challenge. The upside of these experiences is the opportunity to connect the young leader to a wide cross section of people within the organization. Further, this may be one of the first opportunities for an individual to network with people in senior management. In addition, these special projects are most likely "piled on" to normal job responsibilities, offering a new level of time management and work/life skill building. McCall, et al. (1988) suggest that developmental projects or task forces "demand that mangers give up an illusion of mastery and instead use the skills of others to complete the project" (p. 33). Line-to-staff switches occur when managers "were plucked, even pushed into one- or two-year assignments in corporate staff roles. All had been in operational jobs where they were responsible for some bottom line numbers. With the switch to a staff assignment, they were suddenly on alien turf. The managers usually relocated to corporate headquarters and reported to or worked with executives several levels up from them, while struggling with a new technical area" (p. 35 \& 37). Starting from scratch may mean a new product or product line, a new plant, or a new division. As previously discussed, Charan et al. (2001) suggest that each passage requires a new set of skills (new capabilities required to execute new responsibilities), time applications (new time frames that govern how one works) and work values (what people believe is important and becomes the focus of their effort) (p. 8). Naturally, these new skills may be completely different than those needed in their previous capacities.

A number of scholars have discussed the learning that occurs through developmental assignments (e.g., McCall, et al., 1988; McCauley et al., 1994; McCauley 
Eastman \& Ohlott, 1995). For instance, Davies and Easterby-Smith (1984) assert that "managers develop primarily through confrontation with novel situations and problems where their existing repertoire of behaviors are inadequate and where they have to develop new ways of dealing with these situations" (p. 180). Patricia Ohlott (1998) categorizes a number of inherent challenges/situations in developmental assignments. These inherent challenges include: job transitions, creating change, high levels of responsibility, nonauthority relationships and obstacles. These align nicely with the types of developmental assignments listed above.

- Job transitions - Job transitions involve personal change. Unfamiliar situations require leaders to build relationships, learn the business and work through others to be successful. Along with these challenges, leaders feel inherent pressure to succeed in their new roles.

- $\quad$ Task related characteristics

o Creating change - Few people have degrees in change management. However, at times this is what developmental assignments require of the leader. Creating change is setting a new direction for the organization or dramatically reducing the workforce.

o High levels of responsibility - With an increase in assignments comes an increase in responsibility. As a result, individuals find themselves in the spotlight and defined as the winners or losers. The stakes are higher and external pressures can be intense. Because of this, work/life balance may be non-existent and leaders may find themselves on overload.

o Nonauthority relationships - The essence of leadership is influence. Individuals who have the ability to influence and persuade others without using formal power are working from a place of leadership more so than a place of management. As a 
result, developmental assignments challenge individuals to develop these skills so people feel ownership in the process.

- Obstacles - Obstacles are inherent in business and can arrive at a leader's doorstep at any time. How an individual works through these obstacles likely determine success or failure; subordinates, bosses and boards may serve as additional obstacles.

Developmental assignments are a rich resource of leadership development, if reflected upon; otherwise, they are simply assignments in the minds of the participants. In his article Executive Development as a Strategic Tool, McCall (1992) suggests that If senior management believes that leadership is not a critical source of competitive advantage, that the current supply of leadership is adequate for the present and the future, the executive ability cannot be developed, or that the cream rises without any help, then executive development will end up as a showpiece rather than a strategic tool. (p. 31)

To capitalize on developmental assignments organizations should set objectives for development and use these experiences strategically to maximize learning. Initiative architects should be intentional about developmental assignments. By doing so, this cost effective development tool will yield optimal results.

Job rotation is a developmental assignment that fosters "lateral transfers of employees between jobs in an organization" (Campion, Cheraskin, \& Stevens, 1994, 1518-1519). Bennett (2003) defines job rotation as "a planned movement of people between jobs over a period of time and for one or more of a number of purposes" (examples section, para. 1) and describes two specific types of job rotation: withinfunction rotation and cross-functional rotation. A within-function rotation is a rotation at 
a similar level within an individual's current function in the organization. Crossfunctional rotations move an employee into different divisions or units of the organization.

The perceived benefits of job rotation as a leadership development tool focus heavily on the development of the individual. Some assert job rotation aids in the employee's improved skills (technical, business and administrative) and abilities (e.g., Bass, 1990; Bennett, 2003; Cheraskin \& Campion, 1996; Yukl, 2002). In addition, job rotation orients new employees to a wide range of business practices (Cheraskin \& Campion, 1996) and "enhance career development because of the adjustments and knowledge acquisition new jobs require" (Campion, Cheraskin, \& Stevens, 1994, p. 1520). Others suggest that job rotation provides employees with varied experience within an organization which creates generalists with a balanced background (Bass, 1990; Bennett, 2003; Campion, Cheraskin, \& Stevens, 1994; Yukl, 2002) and a number of cross-functional relationships (Bennett, 2003). Finally, job rotation may increase commitment, overall satisfaction (Cheraskin \& Campion, 1996) and offset the experience of a plateaued employee by adding new stimulation to the environment (Campion, Cheraskin, \& Stevens, 1994).

Little research has verified the benefits of job rotation and the literature base is sparse even with its wide use in industries such as banking, technology and healthcare. In addition, this development tool may be difficult to evaluate and calculate a return on investment. However, the link to leadership development is a natural one. For those interested in developing employees, this development tool may be one answer depending on the needs and context of the leadership development initiative. It is relatively easy to 
implement and it is a cost effective way to develop employees. Job rotation can provide employees with a new perspective and cross-functional relationships that could aid in their careers. However, job rotation alone does not "do the trick." It should link to a comprehensive development system for maximum effect.

A third developmental assignment, job enrichment, grew out of the ashes of job enlargement in the early 1960 s. Job enlargement, or horizontal job loading simply meant "diversifying the tasks that an employee carried out to make the work psychologically more attractive and demanding" (Patten, 1977, p. 3). However, in the end, job enlargement did little to increase motivation because, from the perspective of the employee, it was simply more work without reciprocal benefit. As a result, the concept of job enrichment or vertical job loading was introduced. Job enrichment meant that "jobs were enriched by adding tasks that were of greater and lesser responsibility (including supervisory and staff activities such as planning and controlling) to the employee's job; a newer more meaningful job was supposedly created out of vertical job loading" (Patten, 1977, p. 3). As a result, the theory posits that, with more autonomy and an increased level of control, employees have an increased sense of self-efficacy (Parker, 1998). According to Frederick Herzberg, job enrichment serves as a long-term motivator of employees. In a 1968 Harvard Business Review article, Herzberg suggested:

The motivation-hygiene theory suggests that work be enriched to bring about effective utilization of personnel. Such a systematic attempt to motivate employees by manipulating the motivator factors is just beginning. The term job enrichment is an embryonic movement. An older term, job enlargement, should be avoided because it is associated with past failures stemming from a misunderstanding of the problem. Job enrichment provides the opportunity for the 
employee's psychological growth, while job enlargement merely makes a job structurally bigger. (p. 92)

The potential benefits of job enrichment exist in the research. In his research of 58 organizations that implemented job enrichment, Alber (1979) found that job enrichment had a positive effect on quality, resource utilization, operating benefits, absenteeism, and turnover. In their literature review, Pierce \& Dunham (1976) found that job enrichment has generally been associated with employee satisfaction. Pierce and Dunham (1976) found that "it appears from the evidence amassed from these studies that the affective and motivational responses are more strongly related to task design than are the behavioral responses" (p. 87). However, Umstot, Mitchell and Bell (1978) suggest that "the relatively weak link between job enrichment and productivity does not mean that the bottom line is unaffected. Job enrichment results in higher quality, less waste, less turnover and absenteeism, fewer grievances, and a generally more committed workforce" (p. 868-869). Additional research focused on the results found in industry. Two organizations with an important role in these studies were Texas Instruments and AT\&T. At Texas Instruments, assembly time was reduced from 75 hours to 32 hours per unit over three quarters (Janson, 1970). At AT\&T, Robert Ford announced that in its 19 internal studies on job enrichment, nine were "outstandingly successful," one was a complete "flop" and nine were "moderately successful" (Reif \& Luthans, 1972).

Reif \& Luthans (1972) suggest, "like all sound management programs, job enrichment ought to be used selectively and with due consideration to situational variables such as the characteristics of the job, the organizational level, and the personal characteristics of the employees" (p. 36). Although an older concept, and one that in its 
heyday was primarily focused on factory jobs, the thinking behind Herzberg's Motivation Hygiene theory is relevant. At face value, this development tool is easy to implement and a cost effective way to develop employees. However, the research and literature base is mixed in its support.

Another kind of developmental assignment is action learning. Action learning was introduced in England by Professor Reginald Revans in the 1940s. In his early days, Professor Revans concluded that traditional classroom training was insufficient and ineffective. Since its inception, different variations of action learning exist in theory and in practice. However, a commonly held definition may look something like:

Action learning, in brief, is learning from concrete experience and critical reflection on that experience - through group discussion, trial and error, discovery, and learning from and with each other. It is a process by which groups of people (whether managers, academics, teachers, students or 'learners' generally) address actual workplace issues or problems, in complex situations and conditions. The solutions they develop may require changes to be made in the organization, and these solutions often pose challenges to senior management. (Zuber-Skerritt, 2002, p. 114-115)

In his book The Origins and Growth of Action Learning (cited in Edmonstone, 2002), Revans made the distinction between puzzles and problems. Puzzles have solutions containing right and wrong answers. In the words of Heifetz \& Linsky (2002), there is a "technical solution" to the puzzle. Problems, on the other hand, do not have easy answers and, if left untreated, results in escalating issues. Heifetz and Linsky (2002) call these adaptive challenges which are problems that do not have technical solutions. In 
adaptive challenges, there is a gap between the ideal and real and somewhere in the middle is a solution. According to Revans, problems are perfect for action learning.

Once a problem emerges, an action learning "set" of between four and eight people convenes to tackle it. Ideally these teams consist of managers with varied levels of experience and mixed backgrounds (Conger \& Benjamin, 1999; Keys, 1994). Regarding the set of participants, Parkes (1998) underscores the importance of voluntary participation; people who have to be there will not be as productive. Once the set is established, it meets for a period of time, discusses the strategic mandate, determines objectives and discusses the issue - generally working to come up with a solution (Conger \& Benjamin, 1999; Doltlich \& Noel, 1998). To achieve the objectives, it is important that the group create a positive culture with clearly defined ground rules and norms of practice. At times, a facilitator or set advisor may be included who is charged with monitoring the group and its process. In certain instances, the set advisor keeps the group on task and provides education for set members as needed. In other instances, this individual does little more than observe.

In addition to the set advisor, the action learning set may benefit from sponsors who are individuals "who are ready to act on behalf of the firm, should the need to do so arise within the set" (De Loo, 2002, p. 247). This need for a sponsor or champion in upper management pervades the literature (Conger \& Benjamin, 1999; Doltlich \& Noel, 1998; Zuber-Skeritt, 2002). Another constituent of the set is clients. These are people with a vested interest in solving the problem. Once the set has determined a course of action, executives agree to attend an evaluation meeting and review the results obtained 
by the action learning sets (Keys, 1994; Vicere \& Fulmer, 1996). Next, the decision makers determine the level of implementation (Peters \& Smith, 1998).

Conger \& Benjamin (1999) underscore the need for reflective learning throughout the process and assert that "the better designed programs powerfully blend reflective learning experiences with the pressures and deadlines of a significant undertaking" ( $p$. 223). Further, Doltlich \& Noel (1998) suggest that "self-reflection is what distinguishes action learning from normal work" (p. 31).

The objectives of an action learning project vary depending on the task. However, direct and indirect objectives may include: teambuilding, networking, action and reflection, problem identification and problem solving. Advantages for participants are improvement in: strategic thinking ability, understanding of group dynamics, relationships across departments and exposure to organizational challenges.

According to Yukl (2002), few studies evaluate the results of action learning. The studies that have empirically investigated action learning came up with mixed results. However, a number of anecdotal success stories exist (examples can be found in Doltich $\&$ Noel, 1998). As a developmental tool, action learning is a step above simple experience. Smith \& O'Neil (2003) suggest:

It is well known that experience itself is a slippery teacher; most of the time we have experiences from which we never learn. Action learning seeks to throw a net around slippery experiences and capture them as learning, i.e., as replicable behavior in similar contexts and as a source of questioning in differing contexts. (p. 64)

However, if leadership development is a primary objective of the action learning project, the initiative architects should carefully link it to appropriate levels of theory 
both in setting up the project and in reflection throughout the project. Returning to adult development and learning theory, the action learning project should help transform the perspective of participants and offer an opportunity to reflect on the process and outcomes. On balance, it is here where the learning occurs.

Feedback-based development tools increase the level of self-awareness in participants. Some suggest that self-awareness is a natural starting point (e.g., Cacioppe, 1998; Conger, 1992; London, 2002; Yukl, 2002; Van Veslor, McCauley \& Moxley, 1998) of leadership development initiatives. Three common development tools to aid in helping an individual become more self aware are 360-degree feedback, instruments and coaching.

The 360-degree feedback process is a widely accepted tool to help leaders examine the perceptions of their co-workers. Also know as multi-rater or multisource feedback, a 360-degree feedback instrument facilitates feedback from supervisors, direct reports, peers and others working closely with the individual (e.g., customers and vendors). Sometimes, the participant also performs a self-assessment. According to the Center for Creative Leadership, several studies have shown that "360-degree feedback can improve performance and lead to behavior change" (Chappelow, 1998, p. 32).

A 360-degree feedback process necessitates several considerations. These considerations include choosing an applicable instrument, confidentiality, leadership commitment, organizational alignment, an established and well-developed feedback process, follow-up support and a goal setting component to ensure follow-through on the part of the employee. 
An organization that aligns the instrument with its values, standards and goals likely increases the instrument's effect. Alignment of the instrument with these variables not only reinforces organizational values, but also assists in holding individuals accountable to the mission of the organization. Moreover, if executed correctly, the tool aligns the individual's needs with those of the organization as a whole. According to Garavan, Morley, \& Flynn (1997), the instrument should focus on behavior and not merely traits of an individual. The authors suggest that "the instrument should ask raters whether the manager does or does not do something rather than whether the manager possesses some personal characteristics" (p. 139).

Receiving feedback can be a difficult process. Even individuals with a high level of self-awareness may have a difficult time receiving feedback. Therefore, it is extremely important that individuals feel supported throughout the feedback process. Some organizations contract with professional, third party individuals trained in feedback to take on this task. Other feedback sessions are conducted by supervisors - hopefully in a professional and meaningful manner. Turning the feedback into action is the end goal of the instrument. A well-aligned 360-degree feedback instrument helps individuals see where to focus energy and attention. In an effort to capitalize on the experience, leaders ought to assist employees in developing action plans that "become part of an individual's yearly performance plan and are thus linked to performance management" (Davis, 2001, p. 29). Further, organizational leaders should provide individuals with the resources to develop, grow and change behavior.

Finally, McCauley \& Moxley (1996) stress the importance of organizational support for feedback mechanisms. In others words, the organization should be supportive 
and willing to assist individuals in their new desire to change behavior. The system of feedback and development planning should occur at all levels of the organization and everyone should participate in the process. Finally, resources (e.g., coaching, mentoring, and training) should be made available in an effort to support those wishing to develop and grow.

Instruments are a fundamental contribution to an individual's leadership development. An American Management Association (1991) survey on workplace testing determined that 31 percent of organizations responding use assessments to evaluate employees. Another estimate is that organizations spend upwards of $\$ 100$ million a year on instruments (Zemke, 1992). On the path to self-awareness, leaders should be familiar with their styles of learning, confrontation and communication. According to Harland (2003), organizations utilize assessments "to enhance self awareness and self-knowledge, identify strengths and weaknesses and enhance team effectiveness" (p. 286). Hundreds of instruments exist and examine virtually every aspect of an individual's personality.

As development tools, instruments are used for a number of reasons. For instance, assessment centers may use instruments to determine an individual's readiness for management. Other organizations use instruments in the leadership/management development process to aid in self-awareness. Examples of instruments associated with leadership development include: The Myers Briggs-Type Indicator (MBTI), Emotional Competency Inventory (ECI), The Multi-Factor Leadership Questionnaires (MLQ), Leadership Practices Inventory (LPI), and the Leadership Action Profile (LAP).

Executive coaching as a discipline entered the scene in the mid 1980s. Since then, few empirical studies have been conducted. In their article Executive Coaching: A 
Comprehensive Review of the Literature, Kokesch-Kampa \& Anderson (2001) suggested that only seven empirical articles on the topic had been conducted at the time of publishing. Despite this fact, hundreds of articles exist and the industry is still growing. According to Training Strategies for Tomorrow (2003), approximately 10,000 coaches fill the marketplace today and there could be as many as 50,000 worldwide by 2007; and with fees of $\$ 1,500$ to $\$ 15,000$ per day, it is a lucrative business for consultants (p. 17).

The literature on executive coaching comes from three primary sources: business, psychology, and training and development. A number of definitions for coaching exist. One that encompasses different perspectives is:

a helping relationship formed between a client who has managerial authority and responsibility in an organization and a consultant who uses a wide variety of behavioral techniques and methods to help the client achieve a mutually identified set of goals to improve his or her professional performance and personal satisfaction and, consequently, to improve the effectiveness of the client's organization within a formally defined coaching agreement. (Kilburg, 2000, p. 67)

As the industry has grown and developed, different types of coaching have emerged. For instance, Peterson (1996) discuses three distinct forms of coaching: targeted coaching, intensive coaching, and executive coaching. Targeted coaching is a "relatively focused, practical, skills-based approach to coaching that is offered to individuals who are motivated to round out their skill set in one or two key areas" (p. 84). Intensive coaching is a more intensive form of coaching for individuals who, for one reason or another, need to improve their skills and competencies in a short period of time (which can be a tall order). Finally, executive coaching is a form of coaching delivered by 
seasoned consultants who serve as sounding boards and objective observers for organizational executives.

Another perspective is put forth by Witherspoon and White (1996) who divide coaching into three distinct categories: coaching for skills, coaching for performance and coaching for development. Coaching for skills helps individuals acquire specific knowledge, skills and attitudes and tends to have a high level of clarity (they reader may think back to the behavioral approach to adult learning). Coaching for performance assists individuals in performing better in their current roles to correct ineffective behaviors in a reactive or proactive manner; however, it is usually reactive. In this instance, the individual may not see the need for the coaching intervention and goal clarity may be difficult to define. As a result, coaching for performance is a long-term endeavor. Coaching for development focuses on the future; this is coaching conducted in a proactive manner for high potential individuals.

A final distinction is internal vs. external coaching. Internal coaching is conducted by an individual within the organization and is "a one-on-one developmental intervention supported by the organization and provided by a colleague of those coached who is trusted to shape and deliver a program yielding individual professional growth" (Frisch, 2001, p. 242).

A number of benefits of executive coaching exist. First, the term coaching implies a long-term and in-depth relationship as opposed to a one-off seminar or classroom experience (Niemes, 2002; Tobias, 1996). In addition, coaching meets the needs of the individual. It is not a "carpet-bomb" approach to leadership development. As a result, the intervention can meet the specific needs of individuals (Niemes, 2002; Tobias, 1996; 
Witherspoon \& White, 1996). Moreover, a coach can offer individuals an opportunity to stay focused on their areas of attention. This constant focus assists the individual in developing new habits and ways of being (Tobias, 1996). A final benefit is that the learning is of immediate relevance to the individual being coached (Bennett, 2003; Niemes, 2002).

On the negative side, coaching has few industry standards. As a result, few regulations regarding standards, qualifications and ethics exist (Brotman et al., 1998; Kokesch-Kampa \& Anderson, 2001). As Brotman et al. (1998) suggest, "Psychologists have a duty to define the competencies required to achieve sustained behavior through the medium of executive coaching and to be proactive in conveying these standards of competence to the public" (p. 45). In a similar vein, there is concern as to the thin line between coaching and psychotherapy. As a result, some have apprehension about the qualifications one should possess to serve as an executive coach. A number of individuals assert that executive coaches should have knowledge in psychology, business, and adult development as well as politics (Kiel et al., 1996; Laske, 1999; Levinson, 1996; Tobias, 1996). In addition, Training Strategies for Tomorrow (2003) suggests that it may be difficult to maintain a balance between psychotherapy and coaching; "it is not always easy to differentiate between a problem executive and an executive with a problem" ( $\mathrm{p}$. 19). Further, there is little empirical research to support the perceived benefits and drawbacks of coaching (Kokesch-Kampa \& Anderson, 2001; Kilburg, 1996). London (2002) suggests that a "scorecard" outline goals and concrete outcomes so that executive coaches may display their value and effectiveness to the organization. 


\section{Evaluation}

The evaluation of leadership development initiatives is a challenging endeavor, especially when utilizing a number of different tools to assist in development. However, when evaluating development programs and whole systems of programs, the real goal is to find a causal link between initiative objectives and behavior change or "development."

Curriculum designers utilize different evaluation methods depending on the objectives. For instance, some measure skill building activities through direct observation or production numbers. Likewise, action learning projects may yield concrete results based on the outcomes of the project. Kegan and Lahey (19994) measure cognitive development through a method known as the "subject-object" interview. The "subjectobject" interview is a qualitative method based on an interview that measures the cognitive development of individuals. Naturally, competencies such as empathy or complex decision making are more difficult to measure and quantify. However, instruments such as 360s measure the perceptions within an individual's sphere of influence. Over time, improvement in can be measured. However, it is not an exact science.

Therefore, depending on the objectives, there is a need for quantitative and qualitative evaluation of leadership development initiatives. Including both systems of measurement provides a more holistic evaluation for stakeholders, participants, educators and architects of leadership development initiatives. As the task becomes more complex or a "higher order" activity, it is generally more of a challenge to measure.

The cardinal rule of evaluation is that it is planned for at the beginning of the process. Evaluating the effect of leadership development initiatives "after the fact" 
simply will not work (Waagen, 1999). Evaluation should be incorporated into the design of the program/development tool for three primary reasons. First, those individuals funding the initiative may want to know the effect of the training intervention. Second, evaluation can assist architects of leadership development initiatives in measuring their effect on the participant. Finally, Kirkpatrick (1994) suggests that those who evaluate will more likely keep their jobs, improve the overall quality of deliverables, please those in upper management and, potentially, obtain additional funding.

Even though evaluation has a number of benefits, it often takes a back seat to other priorities. Abernathy (1999, p. 21) suggests several reasons evaluation fails in organizations; these reasons include: lack of planning, lack of sponsorship, lack of budget, incompetence, and lack of valid measurements. Generally speaking, working through all of these barriers is a complex undertaking. Along with the above-mentioned barriers, some assert that leadership simply cannot be evaluated. Returning to Jay Conger, he suggests (1992) "the value of leadership is difficult to measure. The answer is that you cannot" (p. 190). However, others suggest that leadership development can be measured and doing so, simply means calculating return on investment through a series of equations (Casico, 1991). Although many models for evaluation exist (e.g., Patton, 1997; Grove, Kibel \& Hass, 2005) the scope of this section is primarily limited to Kirkpatrick's (1994) model of evaluation.

Perhaps the best known model for evaluating training \& development is Kirkpatrick's four levels framework. The four levels determined by Kirkpatrick (1994) include: (1) participant satisfaction/reaction, (2) learning, (3) transfer of behavior and (4) 
business results. Phillips (1994) introduced a fifth level known as return on investment (ROI).

Phillips (1994) recommends the following as target levels for evaluation.

- Level one - Participant satisfaction/reaction 100 percent

- Level two - Learning 70 percent

- Level three - Transfer of behavior 50 percent

- Level four - Business results 10 percent

A major benefit of this model is that, if done correctly, it includes both quantitative and qualitative data. Quantitative data can reveal some of the specific statistics, while qualitative data allows evaluators opportunities to incorporate narratives and stories into the final report.

Level one of Kirkpatrick's model is reaction. At this level, the trainer is concerned with the participant's reaction and the stakeholders' satisfaction with the program. Kirkpatrick (1994) suggests that "it is important not only to get a reaction, but a positive reaction...if participants do not react favorably, they probably will not be motivated to learn. Positive reaction may not ensure learning, but negative reaction almost certainly reduces the possibility of its occurring" (p. 22).

According to Phillips, Wright \& Pettit-Sleet (2000), data from level one evaluation can determine: participant satisfaction with the training, strengths and weaknesses of the training, if objectives were met, the abilities of the presenter, if the appropriate audience was reached and marketing for future trainings. Prior to training, objectives are established so relevant data is collected from participants. Evaluation architects may consider a number of factors when constructing the instrument. First, the 
evaluation may ask a number of forced choice questions represented through quantitative data. A second factor is rater anonymity which fosters an increase in honest reactions to the event. Third, the evaluation architect may include one or more open-ended questions for feedback, especially if a participant had a strong positive or negative reaction. Fourth, the instrument length should be no more than 10 questions (Phillips, Wright \& PettitSleet, 2000). Finally, Phillips, Wright and Pettit-Sleet (2000) incorporated planned action which is "important data (that can be) ultimately used in ROI calculations...participants are asked specifically how they plan to use the program material and the results they expect to achieve. They are asked to convert their accomplishments to an annual monetary value to show the basis for developing the values" (p. 11-12).

Most evaluations are of the above-mentioned nature and simply gauge the reaction of participants (Silberman, 1998). It is the easiest form of feedback to obtain and trainers gain immediate perspective on the experience of the learner. Further, level one evaluation may offer an opportunity to discover the perceived usefulness on the part of the learner. Level two, takes this a step further and measures the learning that occurred.

Level two of Kirkpatrick's model involves the amount of information learned in a given experience. For example, a trainer may subject participants to pre- and post-tests to determine the level of knowledge attained by participants. Level two evaluation is a quick way to see if strategies for teaching participants are working. Kirkpatrick (1994) defines learning as "the extent to which participants change attitudes, improve knowledge, and/or increase skill as a result of attending the program" (p. 22). Evaluation at this level helps examine a participant's ability to understand, comprehend, apply and even synthesize 
information. At this level, it is extremely important that course objectives and testing criteria align.

Some propose that it is not appropriate to evaluate all programs at level two. However, Kirkpatrick (1994) offers the following guidelines for evaluating learning: "use a control group if practical; evaluate knowledge, skills and/or attitudes both before and after the program. Use a paper-and-pencil to measure knowledge and attitudes, and use a performance test to measure skills; get a 100 percent response; and use the results of the evaluation to take appropriate action" (p. 43). Now that participant satisfaction and learning is measured, Kirkpatrick's model turns transfer of learning into action.

Level three evaluation involves the change of behavior following a training intervention. Level three may also measure the implementation of new behaviors. According to Phillips, Jones, and Schmidt (2000), learning does not transfer to the job in 90 percent of cases. If true, this is a staggering number for those involved in leadership development. When deciding which programs warrant level three evaluation, Phillips, et al. (2000) suggest the following criteria for evaluation: (1) level three evaluation has been planned; (2) the program is one of high visibility; (3) the program is integral to meeting business objectives; (4) behavior change is an essential for the organization; and (5) a large amount of money is spent to conduct the program.

Collection methods for level three may include follow-up surveys or questionnaires, follow-up interviews, follow-up focus groups, on-the-job observation, and action plans. In addition, surveys and questionnaires are a quick and easy way to gather follow-up information. Online collection methods such as www.zoomerang.com or www.surveymonkey.com are generally the most cost effective and efficient way of 
collecting level three information. Survey or questionnaire content should focus on progress on objectives, action plan implementation, relevance of the program, use of program materials, knowledge or skill enhancement, skills used, improvements linked to the program (and potential monetary value), barriers and suggestions for improvement (Phillips, et al., 2000).

A more in-depth approach to level three evaluation is a follow-up interview. A benefit of this approach is the depth of information and opportunity for follow-up questions. Further, this approach allows for additional flexibility and data gathering; additional information is discovered depending on the conversation. For instance, the interviewer has an opportunity to ask questions such as, "Could you provide additional information?" or "What made you feel this way?" These questions are not realistic in traditional surveys or questionnaires. However, Kirkpatrick (1994) suggests that "interviews are very time consuming, and only a few can be conducted if the availability of the person doing the interview is limited. Therefore a small sample of those trained can be interviewed" (p. 56). A variation of the follow-up interview is a focus group which gathers a number of former participants. This approach allows six to 12 participants the opportunity to provide feedback at one time. However, this approach may call for a skilled facilitator who is adept at facilitating a group and maneuvering the dynamics inherent in this process.

On-the-job observation is an additional way to ensure level three evaluation. For instance, in the case of CPR training, a participant should pass a written examination as well as a demonstration. This demonstration shows a transfer of learning has occurred. Level three evaluation may also occur through the use of videotaping, computer 
monitoring, simulation, and supervisor assistance once the participant has returned to work. A final method of level three evaluation is action planning. Action planning helps participants develop an implementation plan for new behaviors and skills. Action plans should be detailed and concrete in nature. An individual evaluating at level three may check in with participants at varied intervals to gauge progress. These plans are strengthened if an individual's supervisor helps ensure compliance and implementation.

Data collection at this level is challenging for five reasons. First, data collection differs for each situation; no templates exist. Second, it is time consuming and can become costly to implement. Third, level three requires the collaboration of the participants (and potentially their supervisors) after the course is complete (Dixon, 1996). Former IBM Chief Training Officer Jack Bowsher (1998) shares two other reasons cited for a lack of evaluation at this level: the fast pace of business and the sheer number of factors to be evaluated.

Assuming participants enjoyed the experience, learned in the classroom and transferred these behaviors, it is important to review how the intervention has affected the business. The fourth level in Kirkpatrick's framework is business impact. It is here that the trainer should show the cause and effect of the training; as a result of " $\mathrm{x}$ " intervention, "y" occurred.. Kirkpatrick (1994) offers the following guidelines for this level of evaluation. He suggests that trainers make use of a control group, allow time for results, measure both before and after the program, conduct a cost-benefit analysis and be satisfied with evidence if solid proof is not obtainable. Kirkpatrick also asserts that "in a court of law, juries are asked to determine if the defendant is guilty beyond a reasonable doubt...The situation is similar when we evaluate results. We look for evidence beyond a 
reasonable doubt that the results occurred because of a training program” (p. 68).

However, Phillips and Stone (2000) disagree.

Phillips and Stone (2000) propose that three questions be answered prior to embarking on evaluation at this level.

- Can data be obtained that stakeholders would regard as evidence of business results?

- Is the program capable of creating an impact on measurable results?

- Can the effects of the program be isolated? (p. 2)

Business results divide into hard or soft results. Examples of hard results (data) may include units produced, equipment downtime, tons manufactured, overtime, items assembled, variable costs, product defects, and overhead cost. Examples or soft results (data) may include grievances resolved, violations of safety rules, counseling problems solved, listening skills, frequency of use of new skills, employee complaints and job satisfaction (Phillips, 1996). According to Phillips and Stone (2000), "the most common approach is to monitor hard performance data such as output, quality, cost and time" (p. 5). However, in his book Costing Human Resources: The Financial Impact of Behavior in Organizations, Casico (1991) developed formulas to calculate the financial effect of turnover, absenteeism, sick leave, smoking in the workplace, employee assistance programs, employee attitudes, job performance, and human resource development (leadership development) programs.

Jack Phillips (1991) introduced a fifth level known as "return on investment" or ROI. Return on investment is determined by subtracting the cost of the training from the determined organizational benefits and multiplying by 100. Ideally, the calculation 
determines return on investment for every dollar spent. Gathering baseline data for all items is a necessity. Whether it is customer satisfaction or the number of widgets produced, the architect of the leadership development plan should have this data prior to the program's inception (Pernick, 2001). In certain instances, trainers may want to locate and study a control group as well.

When determining whether or not to utilize ROI, Phillips (1994) asserts an organization should examine its resources (human, time, financial), determine the level of staff expertise, establish the organization's commitment to measurement of its training programs and determine the amount of pressure felt by the program developers to show ROI. Parry (1997) cites four ways to measure training ROI. The first is to measure ROI with hard data. A good number of technical training programs have hard data to support their efforts. A second is through estimates by trainees and their managers. With this approach, learners share their subjective estimates as to the ROI of a given program. Further, asking the participants and their supervisors this question helps assign a number to their perceived ROI. A third method for calculating ROI is through action planning and manager's briefing. At the conclusion of a program, participants develop action plans which are reviewed with their supervisors. Sometime after the training, participants report on their progress and the ROI for the organization.

A variation on ROI is Return on Expectations (ROE). According to Goldwasser (2001), Verizon implemented ROE by closely examining a senior level administrator's expectations for the training and, following the training, revisiting those expectations. Executives then placed a monetary value on the results and used them as "reasonable 
evidence" for the ROI calculation. According to Verizon, parallel ROI and ROE evaluations yield similar results.

An additional method utilized by Spitzer (1999) is a causal chain. Spitzer argues that is difficult to isolate and make the case for a direct "causality." This falls in line with Kirkpatrick's "shadow of a doubt" stance on this level of evaluation. According to Spitzer (1999), a causal chain "maps the indirect relationship between training and organizational impact... it is a critical path that links a training initiative to a final effect on the business" (p. 46). This methodology has distinct similarities to the user-focused theory of action approach of this dissertation described in Chapter Three.

Phillips (1994) outlines a number of challenges associated with evaluating ROI. First, organizations have different definitions of cost standards. For instance, one organization may consider participant time in the cost of the training, while others may not. Another challenge is that the design of the evaluation intervention itself can vary significantly. For instance, pre- and post-testing or the use of control groups may or may not be used, which can diminish the results of an evaluation exercise. A third challenge is the task of isolating the training event as the reason for change in behavior. It is up to interpretation as to whether or not training had an effect. A final challenge is converting results into monetary terms. By definition, this should be the case to show return on investment. However, this can be a challenge when calculating the value of soft skills.

\section{Chapter Two Summary}

Chapter Two served as a general overview of the leadership development literature. The chapter began with a broad overview of the landscape, discussed a few inherent challenges, and then narrowed to focus on five specific areas of leadership 
development: leadership theory, organizational context, adult development and learning, development tools, and evaluation. As suggested, the literature is vast and covers a wide range of topics; however, these five specific areas serve as a backdrop and academic foundation for the proposed study.

A clear gap in the literature is a discussion of the theory of action that guides the development of leadership development initiatives. The implicit theory of action has real consequences. Not only does it link directly the experience of participants, it also determines organizational effect. The purpose of this study was to examine how academic notions of leadership development compare and contrast with the theory of action that guides corporate leadership development initiatives. A secondary purpose was to analyze the process and potential extensions of the user-focused theory of action approach. Within the scope of the leadership development literature, no study has attempted to examine the implicit theories that organizations use when designing leadership development and how these benchmark against recommended attributes found in the literature. Chapter Three lays out the methodology and procedures for pursuing the research question. 


\section{METHODS: CHAPTER THREE}

The purpose of this study was to examine how academic notions of leadership development compare and contrast with the theory of action that guides corporate leadership development initiatives. A secondary purpose was to analyze the process and potential extensions of the user-focused theory of action approach. Chapter One introduced the background, purpose and problem statement. Chapter Two served as a general overview of the leadership development literature. The chapter began with a broad overview of the landscape and narrowed to focus on five specific areas of leadership development: leadership theory, organizational context, adult development and learning, development tools, and evaluation. Chapter Three begins with a discussion situating myself in the research. In this section, I reveal the potential political, social and cultural biases I bring to the study. Case study methodology served as a container and user-focused theory of action approach served as a technique for data collection; both are defined and discussed. Next, I discuss the pilot study and the overall study procedure. Chapter Three concludes with a discussion of methods to address validity, reliability and ethical considerations.

\section{Situating Myself in the Research}

A number of political, cultural and social influences potentially affected my investigation of this topic. I have been studying the literature on leadership development for more than three years. Because of this, I have developed certain biases and perceptions surrounding the topic. For instance, it is realistic to think that I have 
gravitated primarily toward the concepts in the literature that strengthen $m y$ past experiences and perceptions of leadership development. In addition, those concepts or theories that did not connect with my experience base, or others that I do not yet understand, could be placed to the side and not given proper attention or inclusion. Also, I brought political biases to this study of leadership development. For example, I believe leadership can be taught and that organizations are the institutions best positioned to do so. Further, I have a bias against one-off programs not linked to the organization context and think such programs do little to truly affect the individual and the organization over the long-term. As with its financial system, leadership development should weave throughout the organization's fabric for it to truly affect the individual and the organization.

My experience base in industry primarily lies in the not-for-profit sector and this fact may have affected how I viewed the organization with which I conducted research. My experience has been working in mission-driven organizations and the "bottom-line" was rarely a driving force. Moreover, I have to be cognizant that I was seeing only a small portion of the organization as a whole.

From a cultural perspective, a number of factors could have influenced my work. On a micro-level, classmates, professors and the literature of scholars on the topic of leadership development influence my thinking. The fundamental structure of the Ph.D. program at Antioch University has been an additional influence. Another factor that may have affected the study was that I am a white male who resides in the middle-class. As a white male, I experience the world in a different way from individuals of differing economic status, races, sexual orientation, ability and/or gender. I am aware that I am 
relatively inexperienced and blind to concepts such as institutional racism; rightly or wrongly, it simply has not been a part of my reality. An example may be the fact that I did not consciously look for literature on women and leadership development or minorities and leadership development while conducting the literature review. It simply never occurred to me. On a larger scale, the fact that I am a member of Generation X affects my view of the world. The media, technology, the women's liberation movement, the civil rights movement, the divorce rate, and the American paradigm of the world and countless other factors have influenced who I am and what I brought to this study.

\section{Case Study Methodology}

To investigate my topic, I chose case study methodology. Schwandt (2001) defines case study as "a strategy for doing social inquiry...the case is at center stage, not variables" (p. 22-23). Creswell (1998) defines case study as "an exploration of a bounded system or a case (or multiple cases) over time through detailed, in-depth data collection involving multiple sources of information rich in context" (p. 61). Merriam asserts that (1998) "By concentrating on a single phenomenon or entity (the case), the researcher aims to uncover the interaction of significant factors characteristic of the phenomenon. The case study focuses on holistic description and explanation" (p. 29). The following section provides an in-depth description of case study methodology along with a discussion of its inherent benefits and drawbacks.

Reichardt \& Cook (1979) assert that case study is especially effective when looking at a process, which aligns nicely with the question I have proposed for this study. One can view the "process" of case study in two ways. The first meaning of "process" is 
monitoring: "describing the context and the population of the study, discovering the extent to which the treatment or program has been implemented, providing immediate feedback of a formative type and the like" (p. 33) The second meaning of "process" is causal explanation: "discovering or confirming the process by which the treatment had the effect that it did" (p. 33). A large portion of this study focused on the process a corporation uses to develop its leadership development initiative(s). Therefore, case study is a natural fit.

According to Merriam (1998), case study methodology has three primary characteristics which are particularistic, descriptive and heuristic. Case studies are particularistic because they focus upon a single case, phenomenon, suggest of affairs or occurrence. Investigating a case may provide important information or transferable data regarding the phenomenon studied. Second, Merriam suggests that descriptive means that the end product of a case study is a rich, "thick" description of the phenomenon under study. Thick description is a term from anthropology and means "the complete, literal description of the incident or entity being investigated" (p. 29-30). Finally, case studies are heuristic and extend the reader's understanding of the case. Through the research, the author may extend theory, add insight, or name phenomena that were previously unnamed.

Stake (1995) asserts four other characteristics of effective case study methodology; that it is holistic, empirical, interpretive and empathetic. Stake's use of holism has similarity with Merriam's notion of descriptive in that the contextuality of the case is well developed. In addition, Stake (1995) suggests that holism suggests the case is the primary focus of the investigation and is not necessarily a comparison between it and 
other cases. Case study is also empirical. Case study takes place in the field and focuses upon that which can be observed. In addition, "it strives to be naturalistic, noninterventionalistic; and there is a relative preference for natural language description, sometimes disdaining grand constructs" (p. 47). Case study is interpretive and relies on the researcher's intuition as new information and variables present themselves. In ways, its design emerges; the researcher does not begin the research with pre-determined variables for investigation. Finally, case study methodology is empathetic, and provides the reader with a vicarious experience (Stake, 1995). In addition to the four characteristics mentioned above, Stake (1995) asserts that interpretations of observations be immediately validated and there should be a deliberate effort to "disconfirm" personal interpretations of an event. In addition, the report should allow readers to arrive at their own conclusions and not be overly suggestive in its interpretation.

Like all methods of inquiry, case study brings with it inherent benefits and drawbacks. By giving attention to the inherent drawbacks, the researcher has an opportunity to minimize their effect upon the study. Case study methodology can be time intensive on the part of researcher and participants. Finding individuals and organizations comfortable with the time commitment can be a challenge. Next, a question that challenges researchers is: What should I include? How much is too much? At times, case studies can be long, arduous and cumbersome; especially for individuals hoping to make policy decisions based on the work. Conversely, case studies can oversimplify phenomena and lead the reader to inaccurate conclusions - because the case is presented as a "whole" when it is simply a "slice." The researcher should arrive at a balance between the two extremes given the context and purpose of study. 
Because the researcher is the primary instrument of evaluation, a whole host of challenges may exist. First, awareness of personal biases, political orientations, social and cultural influences is a necessity. If these are not at the forefront of the researcher's thinking, they may present themselves in the data gathering, analysis and interpretation phases. Moreover, because case study does not have a formula, a large portion of the investigation depends on the intuition of the researcher. The researcher's skills in interviewing, observing, analyzing and writing all affect the final product. With this responsibility comes potential for mistakes and poor decisions throughout the process of investigation. Along with the above mentioned concerns come issues of ethics, reliability and validity. The researcher should plan for these challenges in the design phase of the study. For instance, a researcher wishing to increase validity may incorporate member checks and peer examination into the design. Likewise, an audit trail may increase the case study's reliability. Finally, situating oneself in the research and working closely with the IRB keeps ethical issues at the forefront of the researcher's mind as the study progresses.

Along with the drawbacks, case methodology affords the researcher a number of benefits. Merriam (1995) asserts that "The case study offers a means of investigating complex social units consisting of multiple variables of potential importance in understanding the phenomenon" (p. 41). In quantitative research, the researcher determines the research variables ahead of time, which inherently allows little room for the emergence of additional variables. Therefore, important variables that have a real effect on the phenomenon could go unnoticed. By nature, case study can result in a rich description of the phenomenon under investigation, a description that quantitative 
methods have difficulty matching. This description may help readers better understand the phenomenon and develop their own opinions, thoughts, theories or ideas. At times, these new thoughts can serve as a springboard for theory-building or future research.

\section{User-Focused Theory of Action Approach}

Case study methodology served as a container for this study and user-focused theory of action approach served as a technique for data collection. This approach requires that practitioners involved in the leadership development initiative (decision makers or those with direct responsibility) express their theory of action. Argyris and Schön (1978) introduced the concept of theory of action. According to Argyris (1997):

Human beings hold two different master designs. The first incorporates the theories humans espouse about dealing effectively with others. The second design involves the theories of action they use (i.e., their theories-in-use). Whenever any issue is dealt with that activates embarrassment or threat, we have found a systemic discrepancy between the espoused theories and the theories-in-use and a systemic unawareness of the discrepancy while individuals are producing it. (p. 10)

Note that Argyris and Schön focused their analysis on the discrepancies that occur between espoused theories and theories-in-use. That is not the intent of the present study. An analysis of how the organization's theory of action for leadership development "in use" is beyond the scope of this study. However, the concept is important, and provides background and a foundation for the reader. Rather, the intent is to uncover an organization's implicit theory of action that guides the development of its leadership development initiative and then benchmark these with existing literature found in Chapter Two. 
To make explicit the theory of action, Patton (1997) suggests that the researcher examine the question "To what extent and in what ways do the processes, activities, and treatments of a program cause or affect the behaviors, attitudes, skills, knowledge, and feelings of targeted participants?" (p. 217). Making the implicit theory of action explicit can be a challenging process because individuals may be unaware of the principles guiding their decision making process. Patton (1997) suggests that

The purpose of thoroughly delineating a program's theory of action is to assist practitioners in making explicit their assumptions about the linkages between inputs, activities, immediate outputs, intermediate outcomes, and ultimate goals. Suchman (1967) called beliefs about cause effect relationships the program's validity assumptions. As validity assumptions are articulated in a means-ends hierarchy, the evaluator can work with intended users to focus the evaluation on those critical linkages where information is most needed at that particular point in the life of the program...In a utilization-focused evaluation, the evaluator works with the primary intended users to identify the critical validity assumptions where reduction of uncertainty about causal linkages could make the most difference. (p. 225)

A large portion of my research involved doing what is described above - helping the practitioners "in making explicit their assumptions about the linkages between inputs, activities, immediate outputs, intermediate outcomes, and ultimate goals" (Patton, 1967, 225). Please note that I am using the terms "outputs" and "objectives" interchangeably. Patton (1997) asserts that the researcher must do at least five things when assisting an organization in uncovering its theory of action:

1. Makes the process of theory articulation understandable.

2. Helps participants be comfortable with the process intellectually and emotionally. 
3. Provides direction for how to articulate espoused theories that participants believe undergrid their actions.

4. Facilitate a commitment to test espoused theories in the awareness that actual theories-in-use, as they emerge, may be substantially different from espoused theories. (Please note that this is not a goal of this study)

5. Keep the focus on this to make the evaluation useful. (p. 223) (Please note that this is not a goal of this study)

As with other methods, advantages and disadvantages exist. One advantage is that discussing a program's theory of action allows practitioners the opportunity to reflect upon core foundations of their leadership development initiative. In addition, the practitioner is a part of the process and has the opportunity to validate the causal linkages as the researcher works to map the theory of action. As a result, the practitioner has an increased level of ownership in the process. Naturally, the approach has disadvantages as well. Patton (1997) suggests that, as practitioners struggle to share their theory of action, they may become defensive or frustrated by the process. An additional challenge is that this approach has no precedents in the study of leadership development. In essence, this study broke new ground in the study of leadership development initiatives.

In this study, I express the theory of action through one visual. The visual expresses the program's theory of action and links validity assumptions with the program's ultimate, intermediate and immediate objectives. Therefore, I first had to determine the organization's ultimate, intermediate and immediate objectives for the leadership development initiative. Next, I worked to clarify the validity assumptions (perceived cause/effect relationships) for each of the objectives. Below (Figure 3.0) is a sample adapted from Patton (1997). 


\section{Sample Theory of Action}

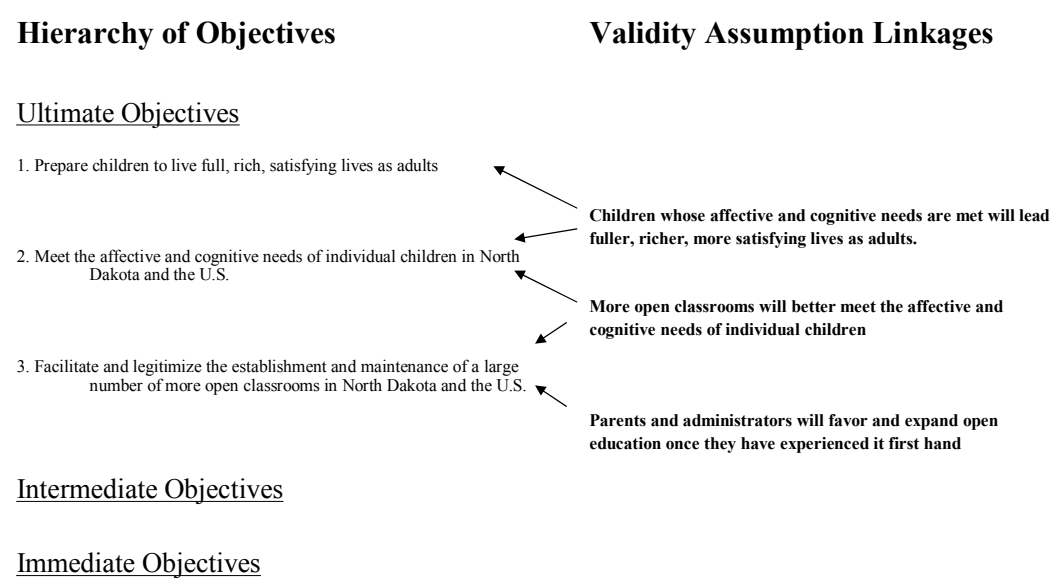

The validity assumptions (right column) link objectives (left column). Arrows indicate to which objectives the assumptions apply.

\section{FIGURE 3.0}

\section{The Pilot Study}

Pilot Study - About the Organization

The pilot study occurred in a 9,400 person organization located in the Northeastern United States; I call the organization "Alpha Company.” The organization helps organizations streamline a number of business processes and was founded in 1971 . Alpha Company has more than 100 offices and serves more than 500,000 clients across the United States. In recent years, Alpha Company has been recognized in a number of “Top 100" lists for their efforts. 


\section{Pilot Study-Process and Procedure}

I gained entrance into Alpha Company through my cousin who is a former employee. He had had a level of involvement with the management development team and made the initial contact. After a preliminary conversation, he provided me with contact information for "Lucy," one of the individuals involved in the five-member management development team. I spoke with Lucy on the phone and also shared the dissertation concept paper. After two conversations, Lucy agreed to participate and located two others closely involved in Alpha Company's leadership and management development. The pilot study design called for two, one-hour meetings with three individuals on two consecutive days. The intent of the study was to practice Patton's methodology (user-focused theory of action) and not to develop a case study. The three study participants were:

- Lucy - A 39 year-old Caucasian female who works on the management development team within the training and development department at Alpha Company. The management development function has two specific roles training and development. Lucy focuses little time on training and a large portion on development or internal coaching and consulting. She is also responsible for a division-wide initiative entitled "Vital-Skills" which focuses heavily on post-modern principles of leadership. Lucy has been with the organization a little more than five years.

- Peter - A 40 year-old Caucasian male who works on the management development team of the training and development department at Alpha Company. Peter has been with the organization more than five years. He spends a large portion of his time in the classroom teaching leadership and management skills.

- Sandy - A 39 year-old Caucasian female who serves as a senior training manager in the training and development department. She has worked for 
Alpha Company for more than 13 years. Prior to becoming a manager, she served on the management development team.

All three met the primary requirements of the study:

- Commitment of time - agree to participation and commit to meeting at least twice over a period of two months.

- Responsibility - have direct responsibility for, or influence on, the leadership development initiative.

- Organizational experience - should have worked in the organization for at least three years to ensure a foundation of corporate knowledge.

I obtained IRB approval and, prior to the research, Lucy, Peter and Sandy each signed an informed consent which read as follows:

"This study examines how academic notions of leadership development compare and contrast with the theory of action that guides corporate leadership development programs. It is performed as partial fulfillment of the requirements for the researcher's (Scott Allen) Ph.D. in Leadership and Change at Antioch University.

As a participant in this study, the researcher will ask you to engage in an interview related to the leadership development program in your organization. The interviews will be about an hour in length and there will be two interviews in all. The interviews will take place on two consecutive days and will be tape recorded for later analysis by the researcher. You will have an opportunity in the second interview to review the researcher's understanding of your ideas as they emerged in the first interview. At the conclusion of the research process, the researcher will be available to each participant to discuss the overall findings of the study. If any quotations from the interviews are used in the final summary, you as the interviewee will be asked to approve their inclusion.

There are no foreseeable risks with this research. The main potential benefit is in contributing to scientific knowledge on leadership development. No costs or payments are associated with participating in the study. If you have any questions about the nature and purpose of this research, the researcher will be happy to answer your questions prior to the beginning of our interview. If at anytime during the interview you feel uncomfortable, you may stop the process and terminate your participation in the study. 
I agree to participate in this research project and I understand that

1. my participation is entirely voluntary. I may terminate my participation at any time without penalty.

2. all responses are confidential and all tape recordings will be destroyed after completion of the study.

3. if I have questions about the research or, if I would like a copy of the aggregate findings of the study when it is complete, I can contact the researcher at sallen@phd.antioch.edu or the supervising faculty member, Professor Jon Wergin at jwergin@phd.antioch.edu.”

The interview format was loose and informal. I determined that leading questions about topics such as evaluation, linkage to HR systems and adult development may cloud what the participant thinks about leadership development. The only two scripted questions were:

- Tell me about your role in the organization.

- Tell me about leadership development at Alpha Company.

The first interview focused on the above questions. However, in reality, participants spent a large portion of time explaining the many leadership development initiatives at Alpha Company. After the initial meetings, I mapped out the leadership development initiative as I understood it and member checked for the first half of meeting two. This proved to be beneficial, because all three had additions to the information. After I gained agreement, I began discussing validity assumptions by asking, "Based upon the model we have outlined, what must be present for all of this to work and live after participants leave your program?" 


\section{Pilot Study - Findings and Learning Moments}

The learning moments ranged in scope from how to use the digital voice recorder to major design flaws discussed later in this section. Please note that the theory of action for each of the three participants is in Appendix B.

As a result of the pilot study, I learned five major lessons. First, two back-to-back, one-hour interviews were not enough to understand the organization adequately, put together a theory of action, formulate its theory of action and discuss validity assumptions. A more realistic approach is to request four 1.5 -hour interviews over a period of two months. The meetings should focus on the following topics.

- Meeting one - In meeting one, I focus on establishing rapport, understanding the individual, their role(s) in the organization, their role(s) in relation to the leadership development initiative, organizational structure, and gaining a broad overview of the leadership development initiative.

- Meeting two - In meeting two, I member check information from meeting one and closely examine the leadership development initiative.

- Meeting three - In meeting three, I member check information from previous meetings and begin discussing validity assumptions based upon the theory of action developed in meetings one and two.

- Meeting four - In meeting four, I bring the three participants together to discuss the overall theory of action. Because there will be differences in responses, this meeting aligns the three perspectives into one theory of action and corresponding validity assumptions.

The second learning moment was understanding that this research should focus on one specific piece of leadership development and not the whole development system. In Alpha Company, I focused on the overall organizational approach to leadership development - making results difficult to obtain. This proved to be too wide a scope for 
this research. For instance, Alpha Company has more than 20 programs running for any number of people (at multiple levels) in the organization. Although similar in scope, I needed more time to understand each program fully and how it fits into the overall picture of development. In addition, some programs do not focus on leadership. Aspects of management development or skills-based training such as budgeting are at the fore. As a result, the findings in Appendix B do not represent fully what I was able to accomplish in the actual research as its scope was narrower.

A third lesson learned is that I need more time between meetings. First, I need a full hour after each meeting to write down thoughts, record comments and capture field notes. In addition, I need at least two days between each meeting to transcribe notes and make sense of the information provided by participants. I felt pressure in Alpha Company because there was not enough time to adequately comprehend and synthesize the massive amount of information.

Fourth, I learned that the participants struggled with the concept of what I was investigating. All three had read the concept paper and I had explained the research but, when repeating the purpose of the research back to me, participants had a difficult time expressing what I was actually doing. For instance, one thought I was looking at "return on investment" and another thought I was trying to prove why it is" impossible to evaluate leadership development initiatives.” In the primary study, I have addressed this issue in the following ways:

- Explain the approach in a way that minimizes academic jargon.

- Use examples to explain the process.

- Ask participants to repeat back to me what I have conveyed.

- Ensure the discussion occurs from the onset. 
Finally, I was amazed at the differences among the three theories of action. For instance, one person was not aware that employees' action plans link to their evaluations; according to one participant, this is even on the organization's official employee evaluation form. In addition, the three individuals had differing perceptions when it came to validity assumptions. One participant, Lucy, focused on the high level assumptions while the other two focused on the lower level assumptions made by initiative architects. As a result, I decided to meet with all three participants in the actual study in a group setting to gain consensus regarding the organization's theory of action and validity assumptions and thus generate one synthesized theory of action.

\section{Primary Study - Procedures}

I have established that leadership development initiatives are prevalent in today's society. Billions of dollars are spent on them and leadership initiatives permeate higher education, not-for-profit foundations and membership organizations. Moreover, I have established that the literature has not investigated how organizations choose to develop their leadership programs. Little is known about the underlying factors that contribute to how these initiatives are developed and, in turn, how these factors benchmark with what has been written on the topic. The central purpose of this research was to examine how academic notions of leadership development compare and contrast with the theory of action that guides corporate leadership development initiatives. A secondary purpose was to analyze the process and potential extensions of the user-focused theory of action approach. As previously mentioned, case study methodology served as a container for 
this study and user-focused theory of action approach served as a technique for data collection.

\section{Sample Selection}

Sample selection occurred on two levels - the case and the individual sample within the case. Both were guided by certain criteria. Case selection was determined in large part by specific guiding principles. First, the organization had made a reasonable commitment to its leadership development initiative. Indicators of "reasonable" were investment of time, financial and human resources. A second variable was that the leadership development initiative had been in continuous existence for three years. This provided a level of perspective as to the program's evolution. An additional criterion variable was the organization's willingness to participate in the study. On an individual level, I sought out organizational leaders who had direct responsibility for leadership development and were willing to set aside the needed time and resources. In addition, I determined that participants should have at least three years of experience within the organization which provided a certain level of institutional knowledge.

Based on the above attributes, I chose to enter Beta Company. Beta Company is located in the Northeastern United States and is known for its "breakthroughs, and cutting-edge technologies... The company ranks as a premier multinational corporation, with a brand recognized in virtually every country around the world" (source: Beta Company web site). The organization has more than 55,000 employees with operations in 30 countries.

I gained entrance into the organization through my uncle who was a former employee. My uncle was in contact with the "Vice President, Human Resources 
Director, Leadership Excellence, Organization Research, Talent Acquisition and Development" (“Ms. Reeves") who, in turn, placed me in contact with an employee ("Oprah") who works directly in leadership development for the company. In early July 2005 , I met with her for one hour to determine if there was a fit between the organization and my study. Oprah discussed the programs within the organization and their Frontline Leadership Excellence System (FLES) seemed to be a perfect match for this research. Following our meeting, I contacted Ms. Reeves to request entrance into the organization. In late July, she approved entrance and secured permission for me to interview her and two other participants directly responsible for the FLES.

Regarding the sample within the case, Merriam (1998) asserts that "since generalization, in a statistical sense is not a goal of qualitative research, probabilistic is not necessary or even justifiable in qualitative research. Thus non-probability sampling is the method of choice for most qualitative research" (p. 61). Researchers should locate a group of participants rich in knowledge and experience - this is "purposive" or "purposeful" sampling. Therefore, I located individuals within the organization who had the following attributes:

- Commitment of time - agreed to participate and commit to 90 minute meetings on four different occasions (note that an exception was made for Ms. Reeves).

- Responsibility - data collection was limited to individuals within the corporation with direct decision making authority over the leadership or professional responsibility for the leadership development initiative.

- Organizational experience - participants worked in the organization for at least three years to ensure a foundation of corporate knowledge. 
In the end, I spent about four hours with two front-line employees and 75 minutes with the Vice President. All three signed the informed consent form (Appendix C), which contained instructions for participants. Informants for the study had the following characteristics:

- Participant \#1 Name: Oprah

Gender: Female

Title: Senior Organizational Effectiveness Consultant

Role: Global 360 Leadership Assessment Coordinator

Years with the Organization: 16 at Beta Company, 3 in current role

- Participant \#2

Name: Lynn

Gender: Female

Title: Director, Leadership Worldwide Center of Excellence

Role: Leads Beta Company's global approach to frontline leadership excellence

Years with the Organization: 21 years at Beta Company, 3 years in current role

- Participant \#3

Name: Ms. Reeves

Gender: Female

Race/Ethnicity: Caucasian

Title: Director Leadership Excellence \& Organization Research, Vice President of Human Resources

Role: Responsible for defining the company's leadership development strategy, enhancing current leadership processes, and the coaching of key executives.

Years with the Organization: 21 years at Beta Company, 3 years in current role

\section{Data Collection}

Data were collected through two primary methods: interview and document review. Person-to-person interviews served as the primary data collection format. Merriam (1998) suggests that "interviewing is necessary when we cannot observe behavior, feelings, or how people interpret the world around them" (p. 72). Given the nature of this study, how participants interpret, define and perceive the leadership development initiative was the primary objective. Stake (1995) suggests that "qualitative 
case study seldom proceeds as a survey with the same questions asked for each

respondent. Rather, each interviewee is expected to have unique experiences, special stories to tell” (p. 64).

Interview questions were semi-structured and changed depending on the purpose of the meeting. The following questions served as guideposts and were developed based upon practice interviews, results of the pilot study and the needs of the study.

- Meeting one

o Tell me about your role in the organization and its structure.

o Tell me about the role of your department.

o Tell me about your role within the department.

o Tell me abut leadership development within the organization.

- Meeting two

o Based on our first meeting, I gathered the following information. I would like to check in with you to be sure I captured everything accurately.

o For the three primary components (360, e-learning, employee development plans)...

- Talk about the thinking and process you used to arrive at this decision to use this as a development method.

- Ideally, how should this activity affect participants?

- What would some indicators be that your efforts are working?

- How was that intended to lead to particular behavior change in participants?

- How should it affect the organization?

- What are the ultimate objectives of the leadership development initiative?

- What benefits will Beta Company supervisors realize from their participation?

- How will you know you have succeeded? How do you know it is accomplishing what it was intended to? 
- What are your thoughts? Benefits? Challenges?

- Meeting Three

o Based on our first two meetings, I gathered the following information. I would like to check in with you to be sure I captured everything accurately.

- Based upon the theory of action I have developed, what factors must be present for each of these pieces to yield the desired results?

- Meeting four (with all three participants)

o As a group let's walk through the theory of action just to be sure we are on the same page.

- Does this activity cause you to think differently about FLES goals and expectations? Where to invest resources for implementation; potential implementation problems?

o As you review the final product, what would some of the next steps be in your mind? What additional steps could be taken to add value to Beta Company?

o How else could an organization use a tool of this nature?

o Do you see a value in a tool of this nature?

- If so, please explain...

- If not, please explain...

o As you reflect on the process we went through (essentially four one-hour meetings), can you think of improvements?

o What other feedback or comments do you have?

Throughout the study, the primary type of question was interpretive. Merriam (1998) defines interpretive questions as providing "a check for what you think you are understanding, as well as provide an opportunity for yet more information, opinions, feelings to be revealed" (p. 78). In ways, this was the most difficult piece of the study - 
asking the right questions, probing when needed, and extrapolating needed information to build a "thick" description.

A final discussion thread on the topic of interviews is the actual interview itself. At the outset, I shared the following with participants through their informed consent:

- The purpose of the research and its goals

- How the information will be used and with whom it will be shared

- The reason they have been selected for participation

- Issues of confidentiality

Additionally, I did my best to provide a comfortable environment with a strong level of rapport. In addition, I was cognizant of remaining neutral regarding the content of the information shared.

Document review or "mining data" was the second data source for this study. In some cases documents were altered to maintain organizational anonymity. Stake (1995) suggests that "gathering data by studying documents follows the same line of thinking as observing and interviewing. One needs to have one's mind organized, yet be open for unexpected clues" (p. 68). The types of documents that contained materials relevant to this study included evaluation instruments, evaluation results, participant materials, facilitator guides, organizational charts, departmental goals and materials relevant to the Frontline Leadership Excellence System (Beta Company's leadership development initiative). Analysis of these documents provided additional assumptions and foundational information regarding the theory of action guiding the leadership development initiative. Merriam (1998) suggests that

Although some documents might be prepared at the investigator's request, most are produced independently of the research study. They are thus, non-reactive and 
grounded in the context under study. Because they are produced for reasons other than the study at hand, some ingenuity is needed in locating documents that bear on the problem and then in analyzing their content. Congruence between documents and the research problem depends on the researcher's flexibility in construing the problem and related questions. (p. 133)

Organizational documents served as secondary to the interviews.

\section{Data Analysis}

Stake (1995) suggests that "perhaps the most important thing is to insist on ample time and space immediately following the interview to prepare the facsimile (report) and interpretive commentary" (p. 66). This thinking was incorporated into the design. All interviews were recorded with the use of a digital voice recorder and transcribed in Microsoft Word format - limited field notes were taken as well.

It is important to note that, throughout the process, different individuals had varying perceptions of the theory of action that guides the leadership development initiative. However, I made the assumption that individuals at similar levels would have similar perceptions. This proved to be the case.

\section{Timeline}

- August, 13, 2005 - Dissertation proposal in Yellow Springs, Ohio COMPLETE

- August 2005 - Secure subject interview times and locations - COMPLETE

- August 2005 - Ask Steve Becker (friend) to copy-edit the first three chapters - COMPLETE 
- September 2005 - Subject interview one (early September) and interview two (late September) - COMPLETE

- October 2005 - Subject interview three (early October) and interview four (late October) - COMPLETE

- November/December 2005 - Complete chapters four and five of the dissertation-COMPLETE

- December 2005 - Gain approval from chair to proceed with formal defense COMPLETE

- December 2005 - Ask Steve Becker (friend) to copy-edit the final two chapters - COMPLETE

- January 2006 - Formally defend the dissertation in Seattle, Washington COMPLETE

\section{Issues of Validity, Reliability, Generalizability (External Validity)}

Issues of validity, reliability and generalizability were addressed and given attention during the design of the study. The following section introduces these concepts and shares how I addressed these potential pitfalls.

Internal validity addresses the level to which research findings match reality. Of course, this definition can lead to a debate as to the meaning of "reality." Regardless of one's position on that question, Stake (1995) asserts that those conducting case study research have "ethical obligations to minimize misrepresentation and misunderstanding. We need certain triangulation protocols or procedures which researchers and readers alike come to expect, efforts that go beyond simple repetition of data gathering to deliberative effort to find the validity of data observed" (p. 109). Efforts to represent the phenomenon accurately are of value. Within the scope of this study, I have chosen two basic strategies for enhancing internal validity. First, I have been clear regarding my 
biases and the cultural, social and political factors that may have an effect on the study (see the situating myself in the research section of this chapter). A second strategy is through member checking. In the process of member checking, participants "are requested to examine rough drafts of writing where the actions or words of the actor are featured, sometimes when written up but usually when no further data will be collected from him or her" (Stake, 1995, p. 115). I took special care to consistently verify and re-verify my interpretation of the organization's theory of action, and participants' comments and quotes.

In traditional terms, reliability refers to the extent to which a research study can be reproduced by another. A number of qualitative researchers suggest that the notion of "reliability" within case study research is fundamentally flawed. Case study research is not conducted so that phenomena can be isolated and replicated. In fact, some argue that rather than looking for consistency, one should be concerned with "whether the results are consistent with the data" (Merriam, 1998, p. 206). I chose one strategy to increase the reliability of the study. An audit is a "procedure whereby an independent, third-party examiner systemically reviews an audit trail maintained by the inquirer" (Schwandt, 2001, p. 8). An audit trial is an opportunity for others to examine how the researcher collected data, arrived at conclusions and designed the study. By nature of the dissertation process, I was provided an audit trial, allowing for all decisions and actions surrounding the study to be closely examined by the dissertation committee.

In addition to internal validity and reliability is the concern for external validity or generalizability. Generalizability is the level to which one study transfers to other situations. For instance, to what extent will the findings of this case study identify similar 
phenomena in comparable organizations? The answer is, "very little.” Qualitative research requires little or no attempt to generalize beyond the case to an assumed population of cases; instead, the case should be potentially transferable to other contexts. Schwandt (2001) suggests that

Case-to-case transfer, an activity that is the responsibility of the reader of research, can be accomplished if the inquirer provides sufficient detail about the circumstance of the situation or case that was studied so that readers can engage in reasonable but modest speculations about whether findings are applicable to other cases with similar circumstances. (p. 107)

Therefore, it is my job to assure that the reader has enough information to engage in speculations about the research and its application to other situations. However, the userfocused theory of action approach may be generalizable and applied to other situations.

The level to which one applies the concepts of validity and reliability directly to qualitative research is a large debate that is not discussed in this dissertation. However, I consciously worked to present clear and accurate data to the reader; strategies such as member checks, audits and peer examination assisted in this endeavor.

\section{Ethical issues}

Ethical practice in research means that "to gain support from participants, a qualitative researcher conveys to participants that they are participating in a study, explains the purpose of the study, and does not engage in deception about the nature of the study" (Creswell, 1998 p. 132). Ethics is a topic that deserves special attention in all phases of the study. 
Research is a ripe arena for the emergence of ethical dilemmas. Whether in the design of the study, the gathering of data or the dissemination of the research, a researcher should work within the guidelines of ethical standards and keep the issue at the forefront of their thinking. Schwandt (2001) asserts:

Inquirers cannot rightly understand their ethics - habits, obligations and modes of thought that shape and define their interactions as social scientists with others without simultaneously thinking through what constitutes legitimate, warranted knowledge of social life as well as what compromises their political commitments and responsibilities as inquirers into the nature and meaning of human affairs. (p.

Situating myself in the research, member checking, the dissertation committee and the institutional review board (IRB) were strategies for maintaining ethical integrity. Two areas of specific importance are the collection of data and the analysis and dissemination of information. For example, within the interview process respondents should decide what kind of information to share and at what level of candor. The information shared may have long-term consequences for them as individuals or their immediate spheres of influence. In addition, participants may have a difficult time putting their feelings, thoughts, ideas and experiences into words. As a result, I focused upon gathering data through non-judgmental and ethical means. The analysis and dissemination of data was another phase deserving of special attention.

\section{Chapter Three Summary}

Chapter Three began with a discussion situating myself in the research and I made transparent the potential political, social and cultural biases I brought to the study. I then discussed case study methodology and its inherent benefits and drawbacks. As 
mentioned, case study methodology served as a container for this study and user-focused theory of action approach served as a technique for data collection. I discussed this approach and then shared the framework and results of the pilot study and the overall study design. Chapter Three concluded with a discussion of methods of addressing validity, reliability and ethical considerations inherent in research.

Chapter Four focuses on the study and the findings therein. I develop a rich description of the leadership development initiative and place the material within the framework of Patton's user-focused theory of action approach. In addition, I make "explicit their assumptions about the linkages between inputs, activities, immediate outputs, intermediate outcomes, and ultimate goals" (Patton, 1997) regarding Beta Company's leadership development initiative, the Frontline Leadership Excellence System (FLES). Next, I provide the reader with seven results, reactions, and findings based on my debriefing meeting with the three participants. Finally, Beta Company's theory of action is benchmarked against the literature discussed in Chapter Two. Implications for practice are discussed in Chapter Five. 


\section{DATA COLLECTION \& RESULTS: CHAPTER FOUR}

The purpose of this study was to examine how academic notions of leadership development compare and contrast with the theory of action that guides corporate leadership development initiatives. A secondary purpose was to analyze the process and potential extensions of the user-focused theory of action approach. Chapter One introduced the background, purpose and problem statement. Chapter Two served as a general overview of the leadership development literature and began with a broad overview of the landscape and narrowed the focus on five specific areas of leadership development: leadership theory, organizational context, adult development and learning, development tools, and evaluation. Chapter Three began with a discussion situating me in the research wherein I made transparent the political, social and cultural biases I potentially bring to the study. I then discussed case study methodology and its inherent benefits and drawbacks. Case study methodology served as a container for this study and the user-focused theory of action approach served as the technique for data collection. I discussed this approach and then shared the framework and results of the pilot study and the overall study design. Chapter Three concluded with a discussion of methods of addressing validity, reliability and ethical considerations inherent in research.

Chapter Four focuses on the study findings and discusses the specific case examined. I explain the organization's theory of action and the validity assumptions. I then focus on participant reactions and thoughts gathered at a debriefing meeting. The chapter concludes with a comparative benchmark of Beta Company's approach with the literature on leadership development. 


\section{The Beta Company Case Study}

\section{Background information}

Over a period of two months, I met with three leaders of Beta Company. The first participant was “Oprah.” She serves as a Senior Organizational Effectiveness Consultant and focuses the majority of her time on the Global 360 Leadership Assessment Process. She has been with Beta Company for 16 years and has spent three years in her current role. The second participant was "Lynn," Director, Leadership Worldwide Center of Excellence. One of her roles in the organization is to lead Beta Company's global approach for supervisors known as the Frontline Leadership Excellence System. Lynn has been with Beta Company for 21 years and has spent three years in her current role. Finally, I spent time with "Ms. Reeves," who is Director of Leadership Excellence \& Organization Research and Vice President of Human Resources. Ms. Reeves is responsible for defining the company's leadership development strategy, enhancing current leadership processes, and coaching key executives. She has been with Beta Company for 21 years and has spent three years in her current role.

I met individually with Oprah and Lynn three times each and had one phone conversation with Ms. Reeves. Data were gathered primarily through interviews. Each of the meetings was roughly an hour in length, and all (with the exception of a phone conversation with Ms. Reeves) were recorded and transcribed. After a draft theory of action was developed, I spent time on the phone with Ms. Reeves to gain her agreement. Following that conversation, I held a debriefing meeting with all three participants to validate findings, investigate their thoughts and reactions to the process, and potential 
extensions to practice. To a lesser degree, data were collected through organizational documents entitled: Beta Company Leadership Center for Excellence; Frontline Leader Capability Development, 2005 “Quality of Feedback”: Frontline Leadership Employee Development Planning Survey; 2006 Beta Company 360 Leadership Assessment Process; 360 Follow-Up Survey; 2005 Leadership Imperatives; Development Path for New Supervisors - Global Guidelines Overview; Beta Company's Leadership Excellence: Global Resources and Approach; and a 8.3.05 Beta Company Organizational Chart.

Beta Company is an organization in transition. The majority of our discussion focused on the current structure; however, throughout the interview process, Oprah and Lynn expressed uncertainty about the future of their roles. The following passage from Oprah highlighted this ambiguity:

We are restructuring as we speak, so that is important for you to keep in mind. I can't tell you a lot about the new organization, but I will tell you the little I know about my future. Lynn's Center of Excellence is going to be disbanded. Lynn's job has been eliminated. However, it does not mean that Lynn has been eliminated; simply the role is eliminated. I am not sure what that means as we restructure the organization. My position has been retained. That doesn't necessarily mean that $I$ will be retained, although I am continuing to operate under the assumption that I will continue to lead the work I lead today.

The interviews were peppered with uncertainty. This restructuring was occurring throughout the organization - so it was not limited to this one department. As a result, I chose to focus on the current structure with the knowledge that the structure could change tomorrow, next week or within the year. 
All three participants have been with the organization for more than 15 years, and a couple of times mentioned the "pendulum swinging." The "pendulum" is the corporate philosophy or approach toward leadership development; all have seen it at both extremes. Lynn described this when she suggested, "You talk about a change in corporate philosophies: When 'Pete Williams' was the president, he was an advocate of training and he established a minimum expectation that every employee would have at least 40 hours of training." Today, the only required development activity at the corporate level is that everyone in management (including frontline managers) has an Employee Development Plan (EDP) which is a learning and development plan. There are a number of capability development resources available, but each individual business within the organization (e.g., Business A, Business C, Display and Components Group) is responsible for determining its own approach to leadership development.

Another foundation of Beta Company's leadership development initiatives are the 2005 Leadership Imperatives developed by the new CEO and disseminated throughout the organization.

1. $\quad$ Drives to Succeed

- Conveys shared vision/strategy - creates and conveys a clear, compelling shared vision and strategy for successful business.

- Increases shareholder value through focus and accountabilityEstablished stretch goals, is accountable, and holds others accountable for achieving aggressive commitments which increases shareholder value. Focuses on solving BIG customer problems that ultimately will deliver value to shareholders. 
- Creates a Winning/Inclusive Environment - creates a leadership team with a "winning and inclusive" environment that stimulates new thinking, creativity and debate.

- Drives a lean organization - Drives a business operation using lean principles; communicates goals, decisions, directions and priorities so that people in the organization know what they have to do.

- Leads Change - Is willing to take risks, challenge the status quo, champion change, and let go of practices that are no longer effective.

- Collaborates Across Boundaries - Works collaboratively across boundaries for the good of the Company; is willing to confront conflict and manage disagreement to solve business issues.

2. Develop Leaders

- Gives/receives feedback and coaching - Seizes every opportunity to provide and receive feedback and coaching.

- Develops diverse successors - Develops a set of successors that is diverse in the broadest sense, for key leadership positions contributing to Beta Company's long-term success.

3. Leads with Values - drives to win in a way that demonstrates Beta Company Values

- Respect for the Dignity of the Individual

- Uncompromising Integrity

- Trust

- Credibility

- Continuous Improvement and Renewal

- Recognition and Celebration

In addition, the 2005 Leadership Imperatives serve as a foundational document for the 360 Leadership Assessment Process and are the focus of an e-learning module. 
The following sections outline the case study. The quotes are selected from more than 120 pages of single-spaced interview transcripts. First, I provide an overview of the organizational structure of Beta Company and, more specifically, the function of the Leadership Excellence and Organizational Research (LEOR) department within which I conducted my research. Next, I provide historical background and detail surrounding Beta Company's Worldwide Leadership Center of Excellence and its leadership system for all frontline leaders - the Frontline Leadership Excellence System (FLES). Finally, I provide a more in-depth examination of the FLES; examining its varied components and boundaries.

\section{Current organization structure}

Beta Company is headquartered in the Northeastern United States and is known for technology. Beta Company is an organization of 50,000 employees in 58 countries. Of the 50,000 employees, about 10 percent serve as frontline supervisors. For this dissertation, I have chosen to focus specifically on programming targeted for this population.

Beta Company is a matrix organization (a hybrid organizational structure), with both individual businesses and regions playing a role in the organization; including learning and development. According to Lynn, regions are "a geographic area. They are locations where we have either marketing and sales, manufacturing, research and development and grouped all of those locations in a way that made sense; both culturally and across time zones." The regions include:

- REGION 1 - European Region (Europe, Africa and Middle East)

- REGION 2 - Latin America Region 
- REGION 3 - Greater Asia Region

- REGION 4 - Japan Region

- REGION 5 - United States and Canada Region

Along with the regions, the organization has several businesses. In ways, the businesses act as independent organizations throughout the world, and are simply responsible for results at year's end. The businesses include:

- Business A

- Business B

- Business $\mathrm{C}$

- Business D

- Business E

- Business F

Each business is led by a senior vice president and all report directly to the $\mathrm{CEO} /$ President. Additional functions within the corporate structure include a chief administrative officer, chief financial officer, chief marketing officer, director of corporate strategy \& corporate business development, chief technical officer, chief diversity officer, general counsel, chief information officer, chief human resources officer, and chief Beta Company operating system officer. The functional group in which I conducted research is human resources, which is led by Senior Vice President "Bill Johnson.” According to Lynn:

We are part of the HR organization. Bill Johnson has a group of people that report to him that are either HR directors for each of the business units or they're the directors for some of the functional groups... and I reside in one of the functional groups. I work for Ms. Reeves who is also vice president in charge of leadership 
excellence and we've split the work around leadership excellence and leadership development into two areas. One is those activities and strategies affects executives and high potential employees and the other is aimed at frontline leaders. So that's the group that I coordinate.

A visual of this reporting structure is below.

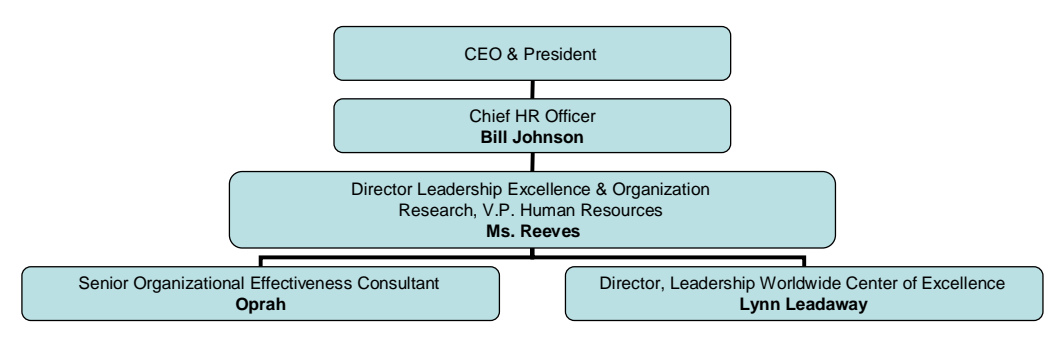

FIGURE 4.0

\section{Leadership excellence and organizational research}

As previously mentioned, the function within which the three participants work is Leadership Excellence and Organizational Research (LEOR). When asked about her area's function, Oprah suggested, "Its strategy, and the actual implementation of that strategy, is to develop leadership excellence in Beta Company's frontline through the 
executive level." Another function of the department is to coordinate the World Wide Leadership Center of Excellence. According to Lynn:

I am coordinating a group of about seven or eight people who are the Center of Excellence (COE). There is a regional leadership development/learning and development professional in each of our regions as well as a couple of other people who are focused on particular aspects of our leadership development strategies. Oprah is part of it - she coordinates all of the 360 Leadership Assessment Process. We also have a guy from our e-Learning Center of Excellence who is part of the group.

Organizational documents say this about the World Wide Leadership Center of Excellence:

We are global network of learning and development professionals positioned to:

- Develop strategies, processes, and initiatives which strengthen Beta Company's Leadership Excellence System and build leadership capabilities across the company, around the world.

- Partner with HR and business leaders to effectively execute plans to help Beta Company "win" in this digital age.

Beta Company has a corporate culture where:

- Leaders are developed through varied channels

- "Great Feedback" is a major development channel

- Leaders are actively involved and accountable for developing other leaders

- Resources that support leadership development are readily available (JIT)

- Leaders are accountable for continual self-assessment and personal development. 
Along with the World Wide Leadership Center of Excellence, Oprah explained, "There's research that's conducted at a corporate level - employee opinion surveys and specific assessment tools that are conducted to help us look at how we are operating as a company." This is another function of the department.

In summary, the Leadership Excellence and Organizational Research group is responsible for developing the corporate strategy for leadership development among frontline and executive leaders of Beta Company. The group is part of Human Resources and, in addition to coordinating development activities, conducts organizational research.

\section{Background on the Frontline Leadership Excellence System}

One output of the Worldwide Leadership Center of Excellence was the Frontline Leadership Excellence System (FLES). When asked about the background of the FLES, both Oprah and Lynn had interesting perspectives on how the FLES came to fruition. Oprah said:

Lynn led a global team (fabulous work). We realized that we needed to improve the capability of our frontline leaders. There was some survey work done to look at people's confidence in their leaders, and we knew we needed stronger leadership - bottom line. There were just a whole bunch of things that indicated that that's where we needed to invest our energy.

Similarly, Lynn said:

Up until 2003, I would say Beta Company, as a corporation, really did not have a complicit corporate approach to leadership development or leadership excellence except at the executive level. So we've always had very strong programs, processes and development venues for executives, or people who are targeted as high potential people but, in terms of new supervisors or second-level supervisors, 
or even third-level supervisors - it was more or less 'sink or swim'....In 2003, Bill Johnson sponsored a global project team to make a recommendation of what Beta Company should do to help improve the consistency of leadership excellence at the frontline level. There were about 16 people on the team from nine different countries and I was the project leader for that. We worked together and basically came up with a recommendation around a Frontline Leadership Excellence System.

\section{Identified components of the Frontline Leadership Excellence System}

The Frontline Leadership Excellence System has a number of components. Lynn summarized these components and the philosophy behind the "system" approach to leadership development when she said, "We got all these great minds around the world together to agree that Beta Company is going to make good progress relative to supervisors and their leadership excellence." The group determined that a simple training module on leadership was not the answer if Beta Company was serious about leadership development. Lynn continued:

We identified elements that needed to be addressed. So, there need to be clear leadership expectations, number one. Then we need to make sure we have a selection process so we are sure we have people with the right kind of capabilities. We need mechanisms to assess leadership effectiveness so that we can provide feedback and coaching - the basis for development. Then, we need development resources in place. In addition, our performance improvement planning process, our performance appraisal process, our employment development process, and pay delivery process all need to be aligned with the expectations. Further, we need a job design that allows people enough time to lead. After all, many times supervisors are also individual contributors so they have major projects and management responsibilities as well. 
In addition, the committee identified the following areas of need: clear behavioral and performance expectations, an understanding of departmental or unit goals, individual goals, the need for a support system (mentor), an understanding of labor laws and corporate policies, how to create a winning and inclusive culture, understanding the performance management process, understanding compensation and benefits, giving and receiving feedback (360 process) and the development of leadership skills. A number of resources support the frontline leader. Lynn explained that the project was "massive," and shared a three-ring binder and $\mathrm{CD}$ that was distributed to frontline supervisors. In addition to the printed materials, Beta Company produced two videos: one explaining leadership expectations, and one about creating an inclusive environment. Today, these resources are located on Beta Company's eCampus and the resources are translated into more than ten languages. Lynn also outlined the ideal "intake" process of a new frontline supervisor:

First of all, if you're a new supervisor, you and your manager would get a note saying 'congratulations' and it would point you to this place on eCampus where you can go to access this development pack. So if you went into our eCampus, you would be directed to go to 'development path for new supervisors' and when you clicked on it, the link would take you to this document which suggests development objectives that should be put in place. For instance, a manager and a supervisor should create objectives for the new supervisor. It also suggests some action items for supervisors and recommends resources (e.g., a mix of e-courses) to help meet the particular development objective.

At the same time these were developed, a set of Minimum Corporate Requirements were created for frontline leaders. Lynn explained that the committee "worked with the HR leadership team to establish the Minimum Corporate Requirements. 
This was the first time that Beta Company had Minimum Corporate Requirements around the globe and we developed some global tools that were disseminated around the world to help organizations meet the minimum corporate requirements." The minimum requirements identified by the committee in 2003 included:

- Clear Expectations

o Align existing leadership competencies with Beta Company's Leadership Competencies for Growth. Help frontline leaders understand what they mean to them on their jobs (now known as the 2005 Leadership Imperatives).

- Effective Supervisor Selection Process

o The interview includes questions related to The Competencies for Growth (now known as the 2005 Leadership Imperatives).

- Leadership Assessment Process

o Potential Supervisor

- Before becoming a supervisor, individuals must complete a self-assessment and debrief it with a "coach."

o Experienced Supervisor

- Organizations will be expected to identify key leadership populations to participate in the 360 Degree Leadership Assessment in 2003 based on business needs.

- Learning and Development

o Potential Supervisors create an understanding of:

- The Competencies for Growth (now known as the 2005 Leadership Imperatives).

- Key roles and responsibilities of a supervisor (so they can make an informed decision).

o New Supervisors

- Create an understanding of The Competencies for Growth (now known as the 2005 Leadership Imperatives).

- The importance of building an "inclusive environment."

o Experienced Supervisor

- Create an understanding of The Competencies for Growth (now known as the 2005 Leadership Imperatives).

\section{- Support Mechanisms}

o All new supervisors (less than 1 yr.) should be provided a form of formal support to help them in their early weeks/months as a supervisor. 
Although identified, the Minimum Corporate Requirements were not fully implemented throughout the organization. As Lynn explained:

There are still Minimum Corporate Requirements and, you might ask, how do you monitor that and make sure it happens? And I would tell you that we were really rigorous about that when we first came up with Minimum Corporate Requirements. However, we've gotten very lax because it takes resources and it takes time. And when you are a company in a huge transition like Beta Company is in...you're changing business models, you're changing the organization structure, you're shutting down operations and you're moving operations. It's not a textbook situation and you do what you need to do...you know? And as a matter of prioritizing and getting your resources or your people to work on your highest priority issues or work... that's one of the things that has just dropped off the plate.

To summarize, the Frontline Leadership Excellence System rests upon the foundation of the Minimum Corporate Requirements and the 2005 Leadership Imperatives. In essence, these foundational documents are guidelines for the new leader. However, for frontline leaders, relatively little accountability is built into the system. The only consistent requirement throughout the organization is that leaders have an Employee Development Plan. The following section discusses the development tools created to help support the FLES.

\section{Development tools}

Leadership development tools are the primary methods for delivering leadership development programming to frontline managers. In the case of Beta Company, the primary development tools utilized at the corporate level are e-learning/eCampus, a 360 
Leadership Assessment Process, and Employee Development Plans. To a lesser extent, the organization is using Leader Learning Labs. These four development tools are Beta Company's Global Platform and are the resources managed by corporate. In the past and in the future, each individual business (e.g., Business C, Business D) determines to what extent these resources are utilized and leveraged. In certain instances, the individual businesses may develop its own set of learning experiences (e.g., coaching and action learning) as well. As a result, what a frontline supervisor experiences in Business C may be different from what is experienced by an employee in Business $\mathrm{F}$ - it all depends. Oprah described the ambiguity well when asked who will be responsible in each business for leadership development - "Resources in the businesses will be dedicated to leadership development. We are embarking on a new model...it isn't clear to me what will be tracked and at what level and by whom - business, region or corporate." Since our initial discussion, it has become clear that, essentially, one person within each business will be responsible for the leadership development of frontline leaders. However, it is likely that the leadership development of frontline leaders will not be their sole responsibility.

Similar to other major corporations throughout the world, Beta Company had a traditional, classroom-based training and development facility in the Northeast. However, in 2004, the facility was sold. According to Lynn, the organization moved to a model of e-learning for a number of reasons:

In terms of e-learning, you're forced to take that route when you've got a global population that you're dealing with. It's just practical and things change so much. For instance, we used to have a corporate orientation class for new employees. However, it's very tough to do something like that globally. Secondly, things change so fast; you get one class completed and, by the next time you're ready to 
conduct it, the whole business model has changed. So, as an alternative to that, we developed an orientation web site, which is updated every few months.

Oprah agreed and added:

e-learning became the new opportunity and there's a lot of cost saving because travel budgets have been restricted. There is also the convenience of doing it when and where you want. For instance, maybe I would like to do it over my lunch hour, over the course of the day, or over the course of five days. Perhaps I want to do it in the evening, or on the weekend or I am in sales and someone who travels and I have time on planes. So we have grown and grown. I'd say most of our learning today is conducted via e-learning.

Currently, the organization develops e-learning courses in-house, and also uses external resources such as OPAL (DDI), Harvard courses and other resources such as SkillsSoft which, according to Lynn, "are suppliers with a whole catalogue of e-based offerings on all kinds of different topics." In general, classroom based training has been all but eliminated (from a corporate standpoint) within Beta Company. Individual businesses may have classroom training but, for the most part, e-learning is the delivery method of choice.

A second development tool, the 360 Leadership Assessment Process, was developed in-house along with a vendor and has been a cornerstone of Beta Company's Frontline Leadership Excellence System. According to Oprah, the nexus of this development tool comes from the fact that “we don't have a very good feedback and coaching culture...everyone will tell you Beta Company is known for being nice, we are comprised of extremely hard working and dedicated people. However, it is not unusual to hear cases where someone had a performance appraisal review, and receives a mixed 
message." Lynn explained that the 360 was chosen because it was well known by committee members and seemed to be the best option based on the needs of the organization.

As mentioned, Beta Company partnered with an external organization and began developing its process. The 360 Leadership Assessment Process is simply a 360 instrument for direct reports, peers/others and supervisors to provide the individual with feedback. According to organizational documents, "If fewer than three Direct Reports responded their responses will be combined with those of Peers/Others. If fewer than three Peers/Others responded their responses will be combined with those of Direct Reports in the Peer/Other line.” Originally, the instrument for frontline leaders was different from those in the executive ranks. However, Oprah suggested, "We are migrating to one Beta Company 360 Leadership Assessment for all leaders in 2006. We will have one site, one system, one assessment tool and one feedback report for all leaders (executive and frontline). The tool will contain 30 items and four open-ended questions."

Along with the 360 instrument, a number of e-learning support resources have been developed. Oprah described some of these when she said, last year we developed a number of online training materials. So there is a 360 training overview on our eCampus, there is a 360 feedback interpretation and delivery module and then there is a 360 follow-up survey overview. These are PowerPoint presentations you can download and they are only available in English at this point; I don't know that we will do any more than that from a resource standpoint. It would be wonderful to have it available in all 10 languages that we administer the 360 process ...I just don't know at the end of the day that we have the resources to do that. 
Turning to the deployment strategy of the 360 Leadership Assessment Process, in recent years, 360s were allocated from the corporate level to the regions by Oprah and her support network. Oprah explained this process in detail when she said:

I had 2000 assessments and had to assess demand, and I had a greater demand than supply so, in the first year, it was based on what percentage of a frontline leadership population resided in that region. For instance, if 80 percent resided in the U.S. and Canada, I gave them an 80 percent allocation of the $2000 \mathrm{I}$ had. And then, as we went through the year, I would reallocate as needed. Our network met on a monthly basis and talked about progress, discussed improvements, tested the instrument and worked through 'localization' issues.

As Beta Company looks to the future, this process and deployment strategy will change - Oprah continued:

We are moving to a business unit deployment strategy. In the previous strategy, I allocated across the regions and then the regions figured out how to allocate them within the businesses. Now, the worldwide business units will establish their own 360 strategies and they make decisions regarding the number of assessments they will conduct globally. The business units will also be accountable for funding their 360 utilization. So it is a huge shift.

With this shift comes a level of uncertainty because, in the future, utilization levels are unclear. Corporate no longer allocates and funds the 360 process. Oprah said, "The assessment is $\$ 50$ per person and the follow-up survey is $\$ 12$ per person, and they (the businesses) will have decision rights on who goes, when, and how often...that's how we are leaving it ...so I think we will see a drop off, but that is okay. People should use it because it makes sense and they value feedback." 
Following the 360 Leadership Assessment Process is a 360 Follow-Up Survey.

This survey is sent to participants in the feedback process six months following the initial run and asks three questions:

1. Did your leader share his/her development actions with you?

2. Do you think your leaders valued and appreciated your feedback?

- Please explain why

3. Have you seen any improvement in his/her leadership effectiveness?

- If yes, please explain what the leader has done to improve his/her leadership effectiveness.

- If no, please explain what the elder can do to improve his/her leadership effectiveness.

A third development tool is the linkage of the FLES to performance management and development planning in particular. In fact, the corporate organization has mandated that all Beta Company supervisors (frontline through executive) have an Employee Development Plan (EDP). For senior executives, the 360 Leadership Assessment Process is mandatory, linked to individual performance appraisals and tied to compensation. However, at the frontline level, the 360 process is voluntary (unless mandated by the individual business) and not tied to an employee's compensation. Lynn explained the process when she said, "That set of processes really falls under the umbrella of the performance management process and that's how we address performance management through performance expectations, performance review and appraisal and then, employee development planning." The manager of the supervisor reviews the employee development plan. When asked about process and follow through regarding EDPs, Lynn 
responded, "It varies. The suggestion is quarterly. I think that probably happens in some areas and in some areas it doesn't."

A final development tool is the Leader Learning Labs. New in 2005, Lynn described the learning labs as an opportunity for supervisors to gather and discuss relevant issues. Similar to open space technology or encounter groups, topics of discussion emerge from the group. As a result, the role of the facilitator is to assist with the process and locate resources as needs arise. Regarding implementation, Lynn explained, "We've got one pilot that we're starting out next month in the Northeast. There's another pilot starting in Germany which is in the planning stage right now. There are also a couple of groups going on in China."

Beta Company's FLES timeline

Worldwide Center of Excellence committee is convened 2002

Competencies for Growth are developed 2002

Frontline Leadership Excellence System is introduced 2003

Minimum Corporate Requirements are introduced 2003

e-learning replaces classroom education as dominant paradigm 2003

360 Leadership Assessment Process are conducted/First round 2003

360 Leadership Assessment Process are conducted/Second round 2004

360 Follow-Up Survey Process is introduced 2004

360 Feedback Training Module is introduced 2004

2005 Leadership Imperatives are introduced 2005 
360 is offline and aligned to fit the 2005 leadership imperatives

Quality of Feedback Survey: Employment Development Planning

As suggested in the introduction, Beta Company is an organization in the midst of change. Along with its changing business model comes a change in how the organization hopes to develop its frontline leaders - placing an increased level of authority within each of the businesses (e.g., Business A, Business B, Business C, Business D, Business E, and Business F).

\section{The Beta Company Theory of Action}

Based on the case outlined, I began to develop the organization's theory of action regarding leadership development at the frontline level. Oprah shared the theory of action in her own words:

You have ongoing development through implementation of your EDP and quarterly development reviews with your manager. Hopefully, throughout, you are utilizing some of these resources. For instance, maybe as a group, you are getting together and looking at some of these things in a meeting but, as an individual, it depends...maybe you are new, and you are going to find a mentor if that is what your region decides to do, or you have been a assigned a group, a learning group, where you get together and talk. Say it's performance assessment (PA) time, and 'gee, none of us have done this so let's do a tutorial about what the PA process looks like, and what the rating scale is, and how you do performance reviews.' So I think your manager works with you, you have the roadmap, you set development objectives, and you may go on eCampus and identify something to 
help you along the way. You may go through the 360 because your entire group goes through it, or you have been identified to go through it....you participate, get your report, review it with your manager, you share your development actions with your direct reports and peers and others from whom you requested input. Then, there would be some developmental actions you take in an effort to close whatever those gaps were and you periodically update people and (in a perfect world), solicit feedback. Then, those who provided you with feedback on your initial 360 would go through the 360 follow-up survey six months after to see if you get some more formal feedback. At this point, you need to determine if you should continue with the 360 . This depends. If you know what you are doing, I guess you can ask for feedback, or if your business is willing to pay for it...then you have time to use the tool, you continue to go through it on some basis. Throughout the process, you will have utilized other development tools in the company as needed, to target the development objectives that you have - they may be financial and have nothing to do with leadership, but this process certainly could identify something besides formal leadership as your challenge. That is what it is intended to do.

Prior to sharing the theory of action, I must remind the reader of a few important concepts. First, the theory of action is a representation of how the participants think their initiative is "supposed" to work. I helped make this explicit along the way but, ultimately, it is intended to reflect their thinking and ideas given the "real life" parameters (e.g., finances, time, location) within which they work. As a result, readers may find their own "unconnected links" or additional steps in the causal chain of events. This is okay and, ultimately, of benefit. However, for this exercise, the result made sense to participants; and, in a holistic way, represented "truth" about their program. This is also true for the validity assumptions. It is highly likely that additional validity assumptions exist. However, the intention is to allow the participants the opportunity to identify some of 
these and "discover" them for themselves. In this study, I presented several validity assumptions to Lynn and Oprah in meeting three; these were eliminated, confirmed or altered. In other instances, assumptions that I had not thought of were suggested and added to the final theory of action.

A final note is that the document reads from the bottom up. The reader should begin on page four and read the entire left side first. This represents the theory of action; how the leadership development initiative is supposed to work. After reading the entire left side, the reader should examine the right side of the equation. The entries on the right side represent validity assumptions. It is helpful to think of these as activities that "have to happen," or be present for the "next step" to occur. All are elements which, if absent, could undermine and/or affect the integrity of the theory.

\section{Beta Company Theory of Action}

\begin{tabular}{|c|c|}
\hline Ultimate Objectives (Corporate Effect) & $\begin{array}{l}\text { Validity Assumptions - What has to happen, or } \\
\text { be present for the "next step" to occur? What } \\
\text { elements could undermine and/or affect the } \\
\text { ability to get to the next level? }\end{array}$ \\
\hline \multicolumn{2}{|l|}{ 16. The business of Beta Company improves. } \\
\hline & $\begin{array}{l}\text { - Leadership capacity drives business results. } \\
\text { - Increased quality of leaders will increase the } \\
\text { chances of business success. }\end{array}$ \\
\hline \multicolumn{2}{|l|}{$\begin{array}{l}\text { 15. Beta Company executives continue } \\
\text { development through Executive Development } \\
\text { Programming. }\end{array}$} \\
\hline & $\begin{array}{l}\text { - Leaders want to continue in leadership positions. } \\
\text { - Leaders succeed in the new level of assignments. } \\
\text { - Participants are legitimately and proactively } \\
\text { involved in their own continual development. } \\
\text { - The metrics (mentioned in 13) can be measured. } \\
\text { - The metrics (mentioned in 13) can be attributed to } \\
\text { leadership development efforts. } \\
\text { - Leadership development efforts will have enough }\end{array}$ \\
\hline
\end{tabular}




\begin{tabular}{|c|c|}
\hline & $\begin{array}{l}\text { time to "take root" in the culture of the } \\
\text { organization before they are changed/dropped. }\end{array}$ \\
\hline \multicolumn{2}{|l|}{$\begin{array}{l}\text { 14. Leaders are better prepared for additional } \\
\text { leadership assignments and enter the pool of } \\
\text { "potential executives." }\end{array}$} \\
\hline & - 13 and 14 are parallel activities. \\
\hline \multicolumn{2}{|l|}{$\begin{array}{l}\text { 13. The company experiences a number of success } \\
\text { indicators: e.g., a decrease in turnover, decreased } \\
\text { level of stress, increased participation in leadership } \\
\text { development, more "solid" managers in the } \\
\text { pipeline, increased competition in the potential } \\
\text { executives category, a decrease in "open } \\
\text { doors," talent is being "poached," people want } \\
\text { to work for certain leaders, good people are } \\
\text { attracting good people from the outside, a } \\
\text { decreased numbers of "checks" in the system, } \\
\text { and an increased level of satisfaction with leaders. }\end{array}$} \\
\hline \multicolumn{2}{|l|}{ Intermediate Objectives (Individual Effect) } \\
\hline & $\begin{array}{l}\text { - A "feedback" culture is valued and helps drive } \\
\text { business results. }\end{array}$ \\
\hline \multicolumn{2}{|l|}{$\begin{array}{l}\text { 12. FLES fosters a culture of giving and receiving } \\
\text { feedback. The } 360 \text { instrument is no longer needed. }\end{array}$} \\
\hline & - 11 and 12 are parallel activities. \\
\hline \multicolumn{2}{|l|}{$\begin{array}{l}\text { 11. Leaders are more effective and more satisfied } \\
\text { in their roles. FLES develops excellent leaders } \\
\text { who help the company succeed. }\end{array}$} \\
\hline & $\begin{array}{l}\text { - Continual development and resource utilization } \\
\text { fosters excellent leadership. } \\
\text { - Development tools meet their needs and foster } \\
\text { growth. }\end{array}$ \\
\hline \multicolumn{2}{|l|}{$\begin{array}{l}\text { 10. Frontline supervisors have a strong grasp of } \\
\text { expectations and are in a process of continual } \\
\text { development. Leaders utilize development tools in } \\
\text { the company as needed. }\end{array}$} \\
\hline & $\begin{array}{l}\text { - A culture exists that values newly learned } \\
\text { knowledge, skills and abilities (KSAs) } \\
\text { - A culture exists that will reinforce newly learned } \\
\text { KSAs } \\
\text { - HR systems (e.g., hiring, management planning, } \\
\text { performance management, job selection, reward } \\
\text { and recognition systems, mistake systems, EDPs, } \\
\text { the immediate supervisor, succession planning } \\
\text { and career development) align with the } \\
\text { development programming }\end{array}$ \\
\hline \multicolumn{2}{|l|}{$\begin{array}{l}\text { 9. Frontline supervisors improve in their } \\
\text { knowledge, skills and abilities (leadership } \\
\text { capacity). }\end{array}$} \\
\hline & $\begin{array}{l}\text { - Supervisors are conducting coaching } \\
\text { conversations and reviewing EDPs - they } \\
\text { prioritize the time and have the skills and abilities } \\
\text { to coach others. } \\
\text { - Resources (time of self/other, human, financial) } \\
\text { are at the disposal of the leader based on his or }\end{array}$ \\
\hline
\end{tabular}




\begin{tabular}{|c|c|}
\hline & $\begin{array}{l}\text { her unique learning needs. } \\
\text { - Supervisors/Participants know where to turn for } \\
\text { support resources within their region or business. } \\
\text { - Supervisors/Participants know how to use these } \\
\text { resources. They are applicable for all employees } \\
\text { (e.g., different languages). } \\
\text { - Participants execute their action plans. } \\
\text { - Participants value learning and development. } \\
\text { - Participants will improve after receiving } \\
\text { feedback. } \\
\text { - Participants are prioritizing/practicing what they } \\
\text { have learned. } \\
\text { - FLES resources are marketed and disseminated in } \\
\text { a consistent and effective manner to all levels of } \\
\text { management. }\end{array}$ \\
\hline $\begin{array}{l}\text { 8. Coaching conversations surrounding } \\
\text { Performance Commitment Planning (PCP), } \\
\text { Performance Appraisal (PA), and Employee } \\
\text { Development Plans (EDP) occur. EDP } \\
\text { conversations occur on a quarterly basis and may } \\
\text { include a special assignment or a special project. } \\
\text { Ideally, these discussions align with the Leadership } \\
\text { Imperatives. }\end{array}$ & \\
\hline \multicolumn{2}{|l|}{$\begin{array}{l}\text { Immediate Objectives (Program } \\
\text { Implementation) }\end{array}$} \\
\hline & $\begin{array}{l}\text { - The minimum expectations are communicated to } \\
\text { all levels of the organization. } \\
\text { - Decision makers prioritize and value elements in } \\
\text { number } 7 \text {. } \\
\text { - Resources - Time, money and human resources } \\
\text { are allocated to accomplish the tasks in number } 7 \text {. }\end{array}$ \\
\hline \multirow[t]{2}{*}{$\begin{array}{l}\text { 7. EDP, Performance Assessment and EDP Quality } \\
\text { of Feedback Survey is required of all frontline } \\
\text { leaders worldwide. Leaders' behavior is consistent } \\
\text { with the leadership imperatives. Frontline leaders } \\
\text { support direct reports who want an EDP and } \\
\text { provide the coaching and feedback they need. }\end{array}$} & \\
\hline & - 6 and 7 are parallel activities. \\
\hline \multirow[t]{2}{*}{$\begin{array}{l}\text { 6. Businesses utilize the chosen resources that they } \\
\text { deem appropriate (internal or external). The } \\
\text { leadership fundamentals are corporately generated } \\
\text { and should be leveraged across the world. The } \\
\text { businesses will develop only that which is uniquely } \\
\text { needed by them. }\end{array}$} & \\
\hline & $\begin{array}{l}\text { - Regions/business/units are supportive and budget } \\
\text { for leadership development expenditures. } \\
\text { - Regions/business/units are supportive and make } \\
\text { development an internal metric for tracking. } \\
\text { - It is clear what metrics will be tracked by each } \\
\text { business. } \\
\text { - It is clear who will have responsibility for } \\
\text { tracking metrics. }\end{array}$ \\
\hline
\end{tabular}




\begin{tabular}{|c|c|}
\hline & $\begin{array}{l}\text { - It is clear what roles the business, region and } \\
\text { corporate will play. } \\
\text { - Everyone understands these roles. } \\
\text { - Each business has a plan for implementation of } \\
\text { development tools and this is communicated to } \\
\text { those who need to hear it. } \\
\text { - Individuals responsible for implementation have } \\
\text { the skills to implement successfully. } \\
\text { - Individuals responsible for implementation have } \\
\text { the financial resources, human resources and time } \\
\text { of participants as well as peers (committees to } \\
\text { help aid in the process). }\end{array}$ \\
\hline \multicolumn{2}{|l|}{$\begin{array}{l}\text { 5. Resources (time, human, financial) in the } \\
\text { business are allocated to leadership development. }\end{array}$} \\
\hline & $\begin{array}{l}\text { - The businesses are in the best position to } \\
\text { determine their individual development needs. } \\
\text { - The regions will understand their role in the } \\
\text { leadership development process. }\end{array}$ \\
\hline \multirow[t]{2}{*}{$\begin{array}{l}\text { 4. Responsibility for the few, common, global } \\
\text { aspects of the FLES is maintained at the corporate } \\
\text { level, while the businesses are given decision rights } \\
\text { relative to how they will address each aspect of the } \\
\text { FLES (e.g., based on business needs, what aspects } \\
\text { they will focus on \& what they will do). The } \\
\text { regions can influence decisions and approach. }\end{array}$} & \\
\hline & $\begin{array}{l}\text { - The three imperatives do, in fact, drive leadership } \\
\text { excellence. } \\
\text { - Beta Company has chosen the most effective/best } \\
\text { development tools for developing leadership } \\
\text { capacity for adults to learn. } \\
\text { - Giving and receiving feedback yields better } \\
\text { results. } \\
\text { - E-learning is an effective way for adults to learn. } \\
\text { - The developmental approach aligns with } \\
\text { employees of differing cultures, ethnicities and } \\
\text { languages. } \\
\text { - People in the management role want to be leaders. } \\
\text { - FLES has been communicated to needed decision } \\
\text { makers to make an educated decision for their } \\
\text { business. } \\
\text { - The "carrot" approach to frontline leadership } \\
\text { development is effective. }\end{array}$ \\
\hline \multicolumn{2}{|l|}{ Program Development and Background } \\
\hline $\begin{array}{l}\text { 3. Resources are developed to support the } \\
\text { minimum corporate requirements of the Frontline } \\
\text { Leadership Excellence System. At the corporate } \\
\text { level, these global resources include: Leader } \\
\text { Learning Teams, the } 360 \text { Leadership Assessment } \\
\text { Process (and follow-up survey) and e-learning } \\
\text { modules (these three sets of resources are known as } \\
\text { the Global Platform). All frontline supervisors are } \\
\text { made aware of Beta Company's } 2005 \text { Leadership } \\
\text { Imperatives - Drives to Succeed, Develops Others }\end{array}$ & \\
\hline
\end{tabular}




\begin{tabular}{|l|l|}
\hline and Leads with the Values. & \\
\hline & \\
\hline 2. The global team identifies the need to create & \\
global expectations \& standards with respect to & \\
leadership expectations (leadership imperatives), & \\
leadership selection, assessment, feedback & \\
\& coaching, leadership development & \\
and performance management (PA/PD, EDP). & \\
Beta Company introduces the Frontline Leadership & \\
Excellence System (FLES) which defines all of the & \\
above mentioned elements. & \\
"Minimum Global Corporate Requirements" & \\
are established and all businesses and regions are \\
expected to comply.
\end{tabular}

The theory of action explained

The following section highlights some important aspects of the theory of action. Please note that text in 'green' denotes the theory of action and text in 'blue' denotes validity assumptions. This is the seventh draft of the document and was validated by all three participants in the debriefing meeting. The document is broken into four primary sections: Program Development \& Background, Immediate Objectives (Program Implementation), Intermediate Objectives (Individual Effect) and Ultimate Objectives (Corporate Effect).

\section{Program Development and Background}

This section of the theory of action is foundational in nature; it represents the background of the Frontline Leadership Excellence System. For example, number one delineates the purpose or reason behind the program's inception:

1. A global team is convened to address a perceived gap in frontline supervisor leadership development within Beta Company. 
Number two identifies the perceived needs for frontline leaders within the organization. What do they need to know as they take on their new role within the organization?

2. The global team identifies the need to create global expectations \& standards with respect to leadership expectations (leadership imperatives), leadership selection, assessment, feedback \& coaching, leadership development and performance management (PA/PD, EDP). Beta Company introduces the Frontline Leadership Excellence System (FLES) which defines all of the above mentioned Elements.

"Minimum Global Corporate Requirements" are established and all businesses and regions are expected to comply.

Number three within this section outlines some of the inputs or development tools utilized to assist with teaching the topics mentioned in number two.

3. Resources are developed to support the minimum corporate requirements of the Frontline Leadership Excellence System. At the corporate level, these global resources include: Leader Learning Teams, the 360 Leadership Assessment Process (and follow-up survey) and e-learning modules (these three sets of resources are known as the Global Platform). All frontline supervisors are made aware of Beta Company's 2005 Leadership Imperatives - Drives to Succeed, Develops Others and Leads with the Values.

For this exercise, I did not identify validity assumptions in the Program

Development and Background section. However, we did identify validity assumptions as we moved from number three to number four, which is the beginning of the implementation phase. I identify these and make comments on a few to help the reader better understand the thought process and reasoning. At this stage, a few crucial assumptions we identified include:

- The three imperatives do, in fact, drive leadership excellence. - the CEO's 2005 Leadership Imperatives do, in fact, develop and drive leadership capacity. 
- Beta Company has chosen the most effective/best development tools for developing leadership capacity for adults to learn. - The architects of the FLES are banking on the fact that the Global Platform (360, e-learning, Employee Development Plan, and Leader Learning Labs) are the most appropriate development tools for employees to learn.

- Giving and receiving feedback yields better results. - By using the 360 Leadership Assessment Process, architects assume that feedback interventions foster employee development, growth, and, ultimately, a positive change in behavior.

- E-learning is an effective way for adults to learn. - Architects are assuming that e-learning is an effective way for adults to learn.

- The developmental approach aligns with employees of differing cultures, ethnicities and languages.

- People in the management role want to be leaders.

- FLES has been communicated to needed decision makers to make an educated decision for their business. - Appropriate marketing and communication has occurred so that the 5000 frontline leaders (and their supervisors) are aware of, and in tune with, the FLES.

- The "carrot" approach to frontline leadership development is effective. - As previously suggested, there is virtually no accountability built into the FLES. Resources are available for use only for those interested. As a result, Beta Company is banking on the fact that this is an effective model upon which the system is established.

\section{Immediate Objectives (Program Implementation)}

The "immediate objectives" section is where initiative architects turn the FLES components over to the organization. Ultimately, it is the implementation phase of the model. Number four in the theory of action rests on the notion that each individual business determines which, if any, of the corporately generated resources (The Global Platform) best meet business needs. In addition, the theory assumes that each of the five regions (e.g., Regions 1-5) will have an influence role in this process.

\section{Responsibility for the few, common, global aspects of the FLES is maintained at the corporate level, while the businesses are given decision rights relative to how they will address each aspect of the FLES (e.g., based on business needs, what aspects they will focus on \& what they will do). The regions can influence decisions and approach.}


Two identified assumptions at this point:

- The businesses are in the best position to determine their individual development needs. - Does each business have an individual who is capable, knowledgeable and in a position to influence and identify an appropriate theory of action for the organization?

- The regions will understand their role in the leadership development process. In the previous model, regions had decision making authority. Now, this responsibility will be eliminated, yet regions may be responsible for implementation of the business' theory of action.

Number five of the theory of action focuses its attention on the resources (e.g., time, human, financial) allocated within each of the businesses. In conversation, both Oprah and Lynn thought that this would vary depending on the organization. However, the amount of time, money and human resources allocated may affect programming within each organization.

\section{Resources (time, human, financial) in the business are allocated to leadership development.}

We made explicit a number of assumptions between four and five of theory of action.

- Regions/business/units are supportive and budget for leadership development expenditures.

- Regions/business/units are supportive and make development an internal metric for tracking.

- It is clear what metrics will be tracked by each business.

- It is clear who will have responsibility for tracking metrics.

- It is clear what roles the business, region and corporate will play.

- Everyone understands the above mentioned roles.

- Each business has a plan for implementation of development tools and this is communicated to those who need to hear it. - Not only is each individual business managing the implementation of their efforts, they are marketing and communicating the efforts to those who need to hear the information within the business.

- Individuals responsible for implementation have the skills to successfully implement. - Each individual business has an individual in place who can effectively manage the FLES process for their organization. 
- Individuals responsible for implementation have the financial resources, human resources and time of participants as well as peers (committees to help aid in the process). - People have the resources needed to succeed.

Implementation within each business occurs at number six of the theory of action.

At the same time, number six is occurring and each frontline leader is participating in the one corporately mandated activity - the Employee Development Activity. Because these occur simultaneously (or close to it), no validity assumptions exist between these two; six and seven are known as "parallel activities."

6. Businesses utilize the chosen resources that they deem appropriate (internal or external). The leadership fundamentals are corporately generated and should be leveraged across the world. The businesses will develop only that which is uniquely needed by them.

- 6 and 7 are parallel activities.

7. EDP, Performance Assessment and EDP Quality of Feedback Survey is required of all frontline leaders worldwide. Leaders' behavior is consistent with the leadership imperatives. Frontline leaders support direct reports who want an EDP and provide the coaching and feedback they need.

For the theory to move to the next level (Individual Effect), these assumptions should be addressed:

- The minimum expectations are communicated to all levels of the organization. The new theory of action is communicated and marketed to all supervisors within the business.

- Decision makers prioritize and value elements in number 7.

- Resources - Time, money and human resources are allocated to accomplish the tasks in number 7.

\section{Intermediate Objectives (Individual Effect)}

The Individual Effect section of the theory of action is where the initiative affects the individual or end user. The theoretical underpinnings exist and the development tools 
exist and have been communicated and implemented within each business. It is here where development of the individual begins. Number eight represents the corporately mandated elements of the theory.

\section{Coaching conversations surrounding Performance Commitment Planning (PCP), Performance Appraisal (PA), and Employee Development Plans (EDP) occur. EDP conversations occur on a quarterly basis and may include a special assignment or a special project. Ideally, these discussions align with the Leadership Imperatives.}

Validity assumptions between eight and nine include:

- Supervisors are conducting coaching conversations and reviewing EDPs - they prioritize the time, and have the skills and abilities to coach others.

- Supervisors/Participants know where to turn for support resources within their region or business.

- Supervisors/Participants know how to use these resources. They are applicable for all employees (e.g., different languages).

- Participants execute their action plans.

- Participants value learning and development.

- Participants will improve after receiving feedback.

- Participants are prioritizing/practicing what they have learned.

- Resources (time of self/other, human, financial) are at the disposal of leaders based on their unique learning needs.

- FLES resources are marketed and disseminated in a consistent and effective manner to all levels of management.

As a result of the activities outlined in number eight, individual leaders begin to grow and develop in their abilities represented by number nine in the theory of action.

Please note that employee development plans are not necessarily "leadership" oriented. Participants may focus on "management" activities such as quality or finances.

9. Frontline supervisors improve in their knowledge, skills and abilities (leadership capacity). 
Between numbers nine and ten, the theory assumes that learning is practiced, valued and reinforced. In the end, unused knowledge will likely be lost during the brain's "pruning process." According to Hoiland (n.d.):

Synaptic pruning eliminates weaker synaptic contacts while stronger connections are kept and strengthened. Experience determines which connections will be strengthened and which will be pruned; connections that have been activated most frequently are preserved. Neurons must have a purpose to survive. Without a purpose, neurons die through a process called apoptosis in which neurons that do not receive or transmit information become damaged and die. Ineffective or weak connections are 'pruned' in much the same way a gardener would prune a tree or bush, giving the plant the desired shape. It is plasticity that enables the process of developing and pruning connections, allowing the brain to adapt itself to its environment (Developmental Plasticity: Synaptic Pruning section, para. 3).

- A culture exists that values newly learned knowledge, skills and abilities (KSAs).

- A culture exists that will reinforce newly learned KSAs.

- HR systems (e.g., hiring, management planning, performance management, job selection, reward and recognition systems, mistake systems, EDPs, the immediate supervisor, succession planning and career development) align with the development programming. - In essence, HR systems help foster a culture of continual leader development and growth.

Number 10 in the theory of action is where individuals within the organization are in a process of continual development and growth.

10. Frontline supervisors have a strong grasp of expectations and are in a process of continual development. Leaders utilize development tools in the company as needed.

Validity assumptions between 10 and 11 include:

- Continual development and resource utilization fosters excellent leadership.

- Development tools meet their needs and foster growth. - employees have access to needed resources that will aid in their development and growth as frontline supervisors. 
As a result of their continual development and growth, the leaders' level of satisfaction increases and it is beginning to affect the organization in a beneficial manner. A parallel activity is that a culture of feedback and coaching exists and, as a result, becomes less of a focus.

11. Leaders are more effective and more satisfied in their roles. FLES develops excellent leaders who help the company succeed.

- 11 and 12 are parallel activities.

12. FLES fosters a culture of giving and receiving feedback. The 360 instrument is no longer needed.

- A "feedback" culture is valued and helps drive business results.

\section{Ultimate Objectives (Corporate Effect)}

Ultimate objectives are the effect on the corporation and its businesses. In theory, it is here where Beta Company begins to see results as an organization. In number 13 , the organization realizes a number of benefits. A parallel activity at this point in the theory is number 14 where there are an increased number of high potentials in the pipeline.

13. The company experiences a number of success indicators: e.g., a decrease in turnover, decreased level of stress, increased participation in leadership development, more "solid" managers in the pipeline, increased competition in the potential executives category, a decrease in "open doors," talent is being "poached," people want to work for certain leaders, good people are attracting good people from the outside, a decreased numbers of "checks" in the system, and an increased level of satisfaction with leaders.

- 13 and 14 are parallel activities.

14. Leaders are better prepared for additional leadership assignments and enter the pool of "potential executives." 
Between number 14 and 15, are a number of key assumptions - especially the ability to link leadership development efforts to organizational effect.

- Leaders want to continue in leadership positions.

- Leaders succeed in the new level of assignments.

- Participants are legitimately and proactively involved in their own continual development.

- The metrics (mentioned in 13) can be measured.

- The metrics (mentioned in 13) can be attributed to leadership development efforts.

- Leadership development efforts will have enough time to "take root" in the culture of the organization before they are changed/dropped. - Beta Company has seen change and its efforts in the area of leadership development have not been exempt from these changes.

Number 15 is an entry into the organization's executive development

programming. The executive development program works under a different model than that of the FLES. For instance, participation in the 360 Leadership Assessment Process is mandatory and linked to compensation. In addition, executives participate in activities such as action learning, succession planning, an executive mentoring process and executive events.

\section{Beta Company executives continue development through Executive Development Programming.}

Between 15 and 16 are some key assumptions:

- Leadership capacity drives business results. - perhaps the largest assumption upon which the theory of action is predicated.

- An increased quality of leaders will increase the chances of business success.

Ultimately, the goal of leadership development at Beta Company is to improve the business. This is a major concern which I discuss in subsequent sections. 


\section{The business of Beta Company improves.}

Once again, the model as described makes explicit Beta Company's theory of action. I worked with participants to build the theory of action and validated its content with all three participants in the debriefing meeting. The following section outlines observations and reactions of participants in the debriefing meeting.

\section{Results, Reactions, and Findings - The Theory of Action}

The following section focuses results, researcher observations, participant perceptions and other key findings based upon a debriefing session held with all three participants. The conversation allowed for emergence of several themes for discussion in Chapter Five.

\section{Making explicit the theory of action}

A primary goal of this research was to work with an organization to make explicit its theory of action that guides a leadership development initiative. There were questions as to whether or not it was feasible to translate Patton's work to this setting. Without question, the user-focused theory of action approach translated to this case and, with relative ease, I was able to articulate the organization's theory of action and validity assumptions surrounding its Frontline Leadership Development Process. In the end, I had consensus of the three participants that, together, we had accurately mapped the theory of action and pinpointed a number of assumptions that, if not addressed, could compromise the integrity of the FLES. 
The process sparked a lively conversation

The process allowed for rich discussion and debate once the three participants were in the same room. Interestingly, each person focused on a different point in the chain, which is discussed in subsequent sections. Because of the nature of the tool, participants had the opportunity to examine the leadership development initiative; its impetus, its implementation, the desired effect on the individual and the desired effect on the organization. On another level, it allowed participants to examine the objectives and goals of the initiative at the individual and organizational levels. In essence, the process maps it all out and makes explicit the assumptions contained in the theory of action.

For instance, the process sparked discussion regarding philosophical challenges one participant had with Beta Company's new approach. At one point, Oprah said:

The interesting thing is our new model has given up some of the things we have a lot of influence or control over. They're operating as independent businesses, making choices based on their needs, prioritizing, and allocating in a way that they believe makes the most sense for them to be successful. There's a whole philosophy behind that approach which doesn't require, or would be inconsistent with, a lot of control mechanisms.

Later in the conversation Oprah said:

I look at the businesses and I think there are a lot of vulnerabilities. I mean philosophically, I have a lot of issues with our whole new structure. We are putting a lot of responsibility and trust in the fact that the leaders in these businesses will make good decisions. 
In another exchange between participants, the following dialogue took place:

Scott: $\quad$...leads with the values. That those (The CEO's 2005 Leadership Imperatives) do, in fact, drive leadership capacity. If they do not, the fundamental foundation may not be there. So, to get from four to five, we are making the assumption that

- The businesses are in the best position to determine what their individual development needs are.

- The regions will understand their role in the leadership development process.

Oprah: $\quad$ Pretty huge assumptions though...if you think about it...

Lynn: $\quad$ Um hum

Ms. Reeves: But they are the assumptions upon which our approach is predicated...

Oprah: $\quad$ Absolutely but, you know, you start to look at it and think, "Do you really think the business is in the best..."

Ms. Reeves: Who knows if the CEO has the right things there (points to the 2005 Leadership Imperatives)?

Oprah: Well, as you asked your question, all I kept thinking was, "We are assuming we are doing the right things..."

Had the focus of the meeting been solely on the findings of the process, I imagine a lively discussion would have occurred. Because this was a research interview, I was forced to reign in these conversations in an effort to stay on track. However, my observation was that participants were less interested in focusing on the questions I was asking because they consistently gravitated to whatever aspect of the theory "spoke" to them. For instance, Oprah returned to the control mechanisms and the overall theory, while Ms. Reeves focused on the bottom line results. 


\section{Multiple levels of discussion}

As previously mentioned, Beta Company is at a challenging time in its history. The business finds itself immersed in an industry that has completely changed in recent years. One of Ms. Reeves' reactions to the process was, "I do think it's useful to put these things in a cascading way and test your assumptions about what really goes on.” In addition, at four points in the meeting, Ms. Reeves returned to the ultimate question of how all of their efforts result in business results. How does all this translate into bottom line results for the business? Sample comments from Ms. Reeves include:

- "Why bother with all the individual programmatic objectives being met if 16 (the business of Beta Company improves) is not accomplished?"

- "So, does the business improve because we have done the right things from a leadership development standpoint? Because we do everything the Top 20 (an annual award given by Hewitt or Mercer) do...we just do not have that (points to number 16 - the business of Beta Company improves)." Perhaps it's an issue of lag time to see the results......and we don't know what the results would have been had we NOT been doing the right things for leadership development.

- "But that is the point of it all...all these things are getting done and yep, supervisors want to be leaders in the future, and we have 360s and all that stuff...and we are getting better...but what is our earnings per share?"

- "Truly, I believe that is, there is all kinds of leadership theory out there that measures this stuff (everything below 16 - the business of Beta Company improves), and that's like the Top 20, right? We are doing some very good things in the Top $20 \ldots$ but we do not have 16 , so what is the difference? 
My impression is that Ms. Reeves' focus on this question was at the forefront of her thinking. Her focus and concern was upon the business results of their efforts. Based on the conversation, my impression was that in her mind, that is the ultimate question. Again, an attribute of this instrument is that, it allows for conversation on different levels - foundational inputs and activities, implementation, individual effect and organizational effect. Ms. Reeves suggests, “That's why our shift on metrics has been from measuring the progress on activities to measuring the ultimate outcomes the activities create. That's the ultimate business goal."

\section{The process sparked informal brainstorming}

The process not only sparked discussions about the theory and its inherent assumptions, but also informal brainstorming by Oprah - a natural extension. For instance, Oprah began thinking of ways to show a return on investment when she suggested:

There's got to be some direct way of measuring that this is impacting business results as opposed to, 'well, we'll just go through these 16 steps and go on blind faith or hope that every step has to have some business impact - some quantifiable business impact - to justify its existence and time, resources, energy invested in it.'

Theory of action as a diagnostic tool \& extensions

All three participants agreed that one benefit of the tool is its ability to serve as a diagnostic instrument for a leadership development initiative. Early in the meeting, Oprah said: 
It would be interesting at some point if you had a diagnostic tool to say 'o.k., these are things that are critical given the structure we have in place, how are we doing?' (Lynn agreed). I am not looking to come up with a complex system, but it would be interesting to say, 'Where are we most vulnerable? Where do we need to do something to ensure that this critical success factor is in place in order for us to be successful?

Later in the meeting Lynn explained:

I do like the tool and I like the methodology from a starting out standpoint making plans and looking at the assumptions that you are making or the critical success factors that have to be in place for you to get to the next step. I like that. And I like it as a debriefing tool to look at what we've done - looking at the assumptions that really needed to be in place and then saying that 'we really started to fall down here and let's see if we can understand why.' So, as a diagnostic tool, I think it's helpful if you want to use it that way...so, in and of itself, I think it's a good tool.

When asked about extension for the user-focused theory of action approach, Oprah said, "We could really complicate it and put in barriers and obstacles as well. The ones that you had to overcome if you are looking at this retrospectively, or you anticipate...you know, what is the show stopper?" Oprah continued later in the conversation:

And then you look at those other things involved and say, 'Which of those can you influence and essentially control?' I don't even think the control word belongs in there, but to what extent can we influence and shape them and have a positive impact on the other things we are working on? To me, we have been very focused on an element, and almost treated it as if it is in isolation as opposed to looking at the whole system and how the pieces fit together... 
The above comments convey their perception of the value of the user-focused theory of action approach as a tool for evaluation of a leadership development initiative. As mentioned in Chapter Three, benefits highlighted by Patton (1997) were that it afforded participants the opportunity to reflect on their programming and be a part of the process - both of these rang true in my experience. The process did, in fact, foster reflection and having participants assist in the process only increased the validity of the resulting document.

\section{Potential for resistance, defensiveness and frustration}

A caution or guideline offered by Patton (1997) is the potential for defensiveness and resistance to this process; especially when you are meeting with the people who developed the initiative under examination. Although I did not face this situation, I can see that the potential exists. After all, the process makes explicit a number of assumptions and gaps in logic. It is clear that some assumptions are out of the direct control of the initiative architects. However, others may not be, and individuals who are not secure with their roles and places in the organization could quite easily feel threatened or insecure throughout the process.

One unexpected reaction came in the debriefing meeting when Oprah expressed, "This has been a very depressing session because, at the end of the day, I don't feel like what we have done makes much of a difference. You know you have to ask yourself, 'Is there something else you could be doing that would have a greater impact?' I don't know what that is..." My observation was that the process left Oprah feeling as though she had 
a minimal effect on the organization and that her efforts had not helped the organization realize its potential. These are important side effects of this process. Organizations do not exist in a vacuum. They are filled with real people with real feelings and reactions to the process. Practitioners face organizational constraints (e.g., financial, geographical) that limit their ability to develop their ideal leadership development initiatives.

\section{No new information}

In the debriefing meeting, Lynn shared an important observation. She was the primary architect of the Frontline Leadership Excellence System and has been with Beta Company for more than 20 years. Her initial reaction to this process was:

Quite honestly, this does not spark any additional insights or thoughts, because it is sort of a recap of our thought process and a description of a path we have taken and assumptions we made along the way. Where something like this might be helpful is if you are beginning the journey and you want to be thinking about the path and all the things you should be thinking about that need to be in place that could increase the likelihood of impact. But you know, I am sitting here thinking about it. Do I look at things differently as a result of this? I don't think I do...

However, later in the same meeting she said:

I do like the tool and I like the methodology from a starting out standpoint making plans and looking at the assumptions that you are making or the critical success factors that have to be in place for you to get to the next step. I like that. And I like it as a debriefing tool to look at what we've done - looking at the assumptions that really needed to be in place and then saying that 'we really started to fall down here and let's see if we can understand why.' So, as a diagnostic tool, I think it's helpful if you want to use it that way...so, in and of itself, I think it's a good tool. 
When used in this environment, the development of additional steps in the userfocused theory of action approach is a necessity. The approach only brings the discussion to a certain point. The tool's power exists when it assists the organization systematically, in not only to identify the assumptions, but address and provide potential solutions - this is discussed in Chapter Five. Perhaps if we had this piece of the equation, Lynn's reaction would have been different. This may be what Ms. Reeves was alluding to when she said:

I think there is huge value in thinking through the causality of one activity to another and what needs to be in place at various steps in the process. So, in that respect, I think it is valuable. I don't think - particularly for staff-oriented initiatives - this activity is rigorous enough.

In summary, the user-focused theory of action approach brought to light some interesting discussions, observations and thinking. Perhaps Oprah summed it up best with the following statement:

It (the activity) just reinforced and brought to light things I have been questioning all along. When you start to see the steps, you are like 'wow.' We are assuming that the businesses are going to know what they need to do, make the good decisions, allocate their resource appropriately and 'course correct.' They're not doing that now! (Lynn agreed). There is lots of hand holding, direction, guidance, and limitation. They (the businesses) are not being given a lot of latitude. There is very little right now. They are being hand held every step of the way, and we are breaking that model at a time when they are not showing that they have earned that privilege. You know what I mean? I am nervous about this. We have been there before in our history when the company did better (Lynn - right, right), and it did not work; they made poor decisions, they spent money inappropriately, 
things got ignored that should not have, so what did we do? We brought it all in corporate and said, 'They are not capable of making these decisions, so we are going to get some Centers of Excellence. We are gonna help.'

\section{Discussion Points from the Literature}

In Chapter Two, I highlighted five primary areas of the literature: leadership theory, linkage to HR systems, adult development and learning, development tools and evaluation. The following section compares and contrasts the literature with the Frontline Leadership Excellence System at Beta Company. Neither the literature on leadership development or Beta Company's practice is viewed as the standard - the purpose of this section is simply to highlight the differences between theory and practice in this case study. Please note that the alignment, moderate alignment and little/no alignment designations are based upon the espoused theory of action not the theory-in -use.

\begin{tabular}{|c|c|c|c|c|}
\hline Literature Topic & & Alignment & Moderate Alignment & Little/No Alignment \\
\hline Leadership Theory & & & & \\
\hline & & & $\checkmark$ & \\
\hline Organizational Context & & & $\checkmark$ & \\
\hline Business Context & & & & \\
\hline Target Population & & $\checkmark$ & & \\
\hline Shared Responsibility & & $\checkmark$ & & \\
\hline Business Systems & & & & \\
\hline Technology & & $\checkmark$ & & \\
\hline Development Plans & & $\checkmark$ & & \\
\hline
\end{tabular}




\begin{tabular}{|c|c|c|c|}
\hline Reward Systems & & & $\checkmark$ \\
\hline Immediate Supervisor & & $\checkmark$ & \\
\hline Hiring & & $\checkmark$ & \\
\hline Succession Planning & & $\checkmark$ & \\
\hline Career Development & & & $\checkmark$ \\
\hline Performance Mgmt. & & $\checkmark$ & \\
\hline Adult Learning & & & $\checkmark$ \\
\hline Transfer of Learning & & & $\checkmark$ \\
\hline Adult Development & & & $\checkmark$ \\
\hline Development Tools & & $\checkmark$ & \\
\hline Dev. Relationships & & & $\checkmark$ \\
\hline Dev. Assignments & & & $\checkmark$ \\
\hline Job Rotation & & & $\checkmark$ \\
\hline Job Enrichment & & & $\checkmark$ \\
\hline Action Learning & & & $\checkmark$ \\
\hline 360 Feedback Process & $\checkmark$ & & \\
\hline Instruments & & & $\checkmark$ \\
\hline Coaching & & & $\checkmark$ \\
\hline Evaluation & & & $\checkmark$ \\
\hline Level 1 & & & $\checkmark$ \\
\hline Level 2 & & & $\checkmark$ \\
\hline Level 3 & & & $\checkmark$ \\
\hline Level 4 & & & $\checkmark$ \\
\hline
\end{tabular}




\section{Leadership theory}

Several theorists assert the importance of having a leadership development initiative rooted in a theory of leadership (e.g., Avolio, 1999; Avolio, 2005; Cacioppe, 1998; Conger, 1992; Conger \& Benjamin, 1999; Goleman, Boyatzis \& McKee, 2002; Popper \& Lipshitz, 1993; Vicere \& Fulmer, 1998). In the case of Beta Company, the organization has its foundation in the 2005 Leadership Imperatives set forth by the CEO:

- Drives to Succeed
o Conveys shared vision/strategy
- Increases shareholder value through focus and accountability
- Creates a Winning/Inclusive Environment
o Leads Change
- Collaborates Across Boundaries

- Develops Others
- Gives/receives feedback and coaching
- Develops diverse successors

- Leads with Values
o Respect for the dignity of the individual
o Uncompromising Integrity
o Trust
o Credibility
o Continuous Improvement and Renewal
- Recognition and Celebration

Aspects of the 2005 Leadership Imperatives closely mirror leadership theory. For instance, Develops Others, Conveys a Shared Vision and Feedback \& Coaching resemble dimensions of transformational leadership theory. Likewise, Credibility, Recognition \& Celebration and Creates a Shared Vision are congruent themes found in Kouzes and 
Posner's Five Practices of Exemplary Leadership. Other components, however, such as Collaborates Across Boundaries, Drives to Succeed, Increases Shareholder Value and Continuous Improvement and Renewal are not mentioned in any theory of leadership of which I am aware. As a result, the three imperatives (and its subsets) are, in part rooted in leadership theory and, in part, rooted in management theory or desired business results rather than theoretical attributes of an effective leader. As a result, it is unclear if the three imperatives upon which Beta Company's FLES exists do, in fact, drive and develop leadership capacity in an individual. Returning to Avolio (2004), "evaluating leadership development interventions is essentially testing the construct validity of the model that underlies leadership development." If this is true, the validity construct of Drives to Succeed, Develops Others, and Leads with Values is called for.

\section{The organizational context}

In Chapter Two, I discussed a culture of leadership development through a supportive organizational context. Returning to Moxley and O'Conner-Wilson (1998), a supportive organizational context includes the business context, target population, shared responsibility and supportive business systems. With the advent of Beta Company's new model, each business is in a position to determine how the organizational context aligns with its leadership development activities for frontline supervisors. In addition, businesses are responsible for deciding the target population for development, the curriculum, the implementation model, evaluation techniques and all other aspects of the leadership development process. If an individual business chooses to utilize corporate resources such as e-learning and the 360 Leadership Assessment Process, it is responsible 
for purchasing them. In sum, the organizational context changes within each individual business.

As a result, each individual business within the organization is responsible for aligning its business systems such as technology, personal development plans, reward systems, the immediate supervisor, hiring, succession, career development and performance management with leadership development efforts. Leadership and adult learning scholars assert that linking leadership development efforts to organizational systems is a crucial component of the transfer of training process. On balance, technology, the immediate supervisor, performance management and reward systems all have a real effect on behavior modification.

\section{Adult development and learning}

Merriam \& Caffarella (1999) suggest five primary orientations to adult learning: behaviorism, cognitivism, humanist, social learning and constructivist. Behaviorism's primary purpose is to elicit behavioral change in a new and desired direction. While behaviorists are concerned with behavioral change, cognitivists focus upon developing “capacity and skills to learn better" (p. 264). Humanists, on the other hand, are primarily concerned with the learner attaining self-actualization and an autonomous, self-directed process to fulfill personal needs. Proponents of social learning examine the intersection of the social context and the learner. Finally, constructivists are concerned with the learners' construction of reality and how individuals make meaning of experiences.

While aspects of adult learning theory permeate Beta Company's Frontline Leadership Excellence System, the architects did not consciously consider adult learning 
theory as it was developed. According to Lynn, the development team relied upon its experience and knowledge of the industry, rather than intentionally turning to theories of adult learning. For instance, a 360 process can help individuals make meaning of their experience, which by nature is valued by constructivists. However, the architects of the FLES did not choose the instrument for this reason. In a similar vein, the architects of the initiative did not seek out theories of adult development theorists either. However, aspects of adult development theory exist in the FLES. For instance, the reflection that coincides with the 360 process aligns nicely with Brookfield's writing on critical reflection. However, these tools were not consciously chosen for these reasons.

\section{Development tools}

The primary development tools used in the Frontline Leadership Excellence System (FLES) are e-learning, the 360 Leadership Assessment Process, and personal development plans. The following section briefly describes the literature on each of these three development tools and how this information aligns with Beta Company.

E-Learning - By all accounts, those interviewed were content with the e-learning process at Beta Company. Oprah and Lynn discussed its benefits for the organization. Elearning is defined as "the use of computer network technology, primarily over an intranet or though the Internet, to deliver information and instruction to individuals" (Welsh, Wanberg, Brown, \& Simmernig, 2003, p. 246). This is an appealing medium and will only grow. For example, in Managing Training and Development's "2004 Training Management and Cost Control Survey" the authors found that 59.2 percent of the organizations surveyed have adopted e-learning and 23.5 percent reported that they plan 
to adopt it. A number of researchers have found that e-learning is an effective means of providing information to adults (Baker, 1992; Brown, 2001; North et al., 2000; O'Hara, 1990). In fact, one study by Kulik \& Kulik (1991) found that technology is slightly more effective than classroom-based training. In addition, a number of studies have shown that learning increases in as much as 50 percent less time (Burns, 2005). A third benefit is that research has concluded that e-learning "can reduce costs if there are a large number of learners, if the learners are geographically disbursed and if the course will be repeated several times" (Welsh, Wanberg, Brown, \& Simmernig, 2003, p. 255). However, elearning may not be for everyone; especially those who are not familiar with technology (Martocchio, 1994; Gist et al., 1989). Moreover, research has found that e-learning may not be appropriate for all learning interventions (Kulik \& Kulik, 1991). For example, an e-learning course in "driver's education" could be of benefit; however, at certain times, a driver needs to actually practice in a car.

360-Feedback - The 360 Leadership Assessment Process at Beta Company is a cornerstone of its leadership development efforts. However, as the organization shifts, corporate will no longer allocate a set number of instruments to its businesses and/or regions. Each business is responsible for registering and paying for its employees to participate. Doing so may increase the instrument's effect on the end user and better facilitate behavior change. The literature is vast; however, in the following section, I highlight research findings that may be of interest to Beta Company.

Also known as multi-rater or multisource feedback, a $360^{\circ}$ feedback instrument facilitates feedback from supervisors, direct reports, peers and others working closely with the individual (e.g., customers and vendors). Sometimes, the individual under 
consideration also performs a self-assessment. According to the Center for Creative Leadership, several studies have shown that "multirater feedback can have a positive impact on individuals" (Chappelow, 2005, p. 67). An organization that aligns the instrument with its values, standards and goals increases the instrument's effect. Alignment of the instrument with these variables not only reinforces organizational values, but also assist in holding individuals accountable to the mission of the organization. Beta Company aligns nicely with this statement and has spent the last year working to align its instrument with its 2005 Leadership Imperatives mentioned earlier in this dissertation. According to Garavan, Morley, \& Flynn (1997), the instrument should focus on behavior and not simply on traits of an individual. The authors suggest that "the instrument should ask raters whether the manager does or does not do something rather than whether the manager possesses some personal characteristics" (p. 139). Here again, Beta Company is in alignment with the literature.

In their meta-analysis, Kluger \& DeNisi (1996) found that, upon receipt of feedback; (1) one third of participants improve; (2) one-third maintains the status quo; and (3) one-third decrease in performance. In addition, the authors found that people internalize feedback depending on their perception of "feedback consequences." For instance, individuals who know that change is expected are more likely to work on their behavior. Maurer \& Palmer (1999) found that three factors affect an individual's decision to make changes following feedback: perceived favorable outcomes, perceived social pressures, and the individual's perceived control over his improvement. Ryan, Brutus, Greguras, \& Hakel (2000) researched recipient characteristics that led to feedback acceptance: self-awareness, age, demographic similarity, acquaintance, and self-esteem. 
The authors found partial support for the notion that those with increased self-awareness would better receive the feedback. In addition, Ryan et al. (2000) found that older individuals were less likely to accept the feedback; however, racial similarity and level of acquaintance partially-increased participant receptivity to feedback. Greguras, Ford, \& Brutus (2002) found that participants in multi-source feedback tend to focus on the supervisor rating, which may be good because Eichinger \& Lombardo (2004) found that an individual's supervisor was the most accurate rater in predicting long-term success. In addition, the largest factor in rater accuracy was "how long the rater has known the person." Moreover, Eichinger \& Lombardo (2004) found that self-ratings often miss the mark and individuals who overrate themselves tend to "fail" and those who underrate tend to "succeed." Finally, Smither, London, Flautt, Vargas, \& Kucine (2003) found that individuals working with a coach following $360^{\circ}$ feedback were more likely to set specific goals, solicit ideas for improvement from their supervisors and receive improved scores in subsequent $360^{\circ}$ evaluations.

The effect of the instrument is dependent upon a number of variables; however, according to some research, one of the most important is the debriefing and feedback process. Realizing the importance of this phase of the process, Beta Company developed a training module for leaders to review prior to debriefing feedback with participants. The effect of the $360^{\circ}$ feedback increases with a solid performance development plan and assistance from a coach. Once again, this development tool in conjunction with others leads to a larger degree of success.

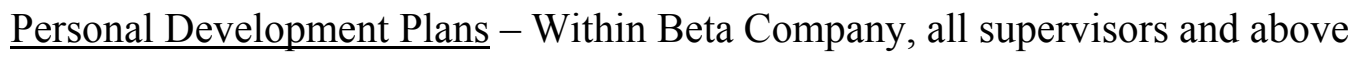
are required to have a personal development plan. The personal development plan is a 
more individualized approach to leadership development and, like other development tools, has potential benefits and drawbacks. Taylor and Edge (1997) define a personal development plan as "a process through which the individual prepares a training and development plan, and for which the individual takes responsibility" (p. 21).

Little empirical evidence exists for the benefit of personal development plans. However, development plans may encourage individual accountability for learning, reinforce the total quality principles of continuous improvement, and translate learning into meaningful and measurable action plans that not only improve the individual, but also the organization (Floodgate \& Nixon, 1994). In addition, development plans may assist supervisors in coaching and developing others and help foster a culture of leadership development at all levels of the organization. In addition, cultural traditions of how people learn are a natural barrier; employees may be unaccustomed to guiding their own development which can be major paradigm shift. As a result, supervisors may not be trained to (or take the time to) follow-up with individuals regarding their development plans. Finally, personal development can be a challenge to administer without the use of technology. Popular in practice, it is cost effective, easy to implement and a "spark" for ongoing and continued development. However, employee development plans have little power and effect without the support of organization leaders and a culture that links it to elements such as performance appraisal and succession planning.

Based on conversations with Oprah and Lynn, I assert that their experience is similar to that of the literature. When a supervisor takes the time to develop and follow through with plans, it can be a powerful experience. However, absent this crucial 
dimension, personal development plans, like other development tools, may not have the desired effect without other organizational systems supporting them.

\section{Evaluation}

There has been no formal evaluation of the Frontline Leadership Excellence System. However, evaluation of certain aspects of the FLES, such as the 360 Leadership Assessment Process, occurs on a few levels. For instance, in a follow-up survey, 80 percent of the participants perceived a positive change in the behavior of their leader. However, other than anecdotal feedback, it is unknown as to the overall effectiveness of the e-learning, personal development plans and Frontline Leadership Excellence System as a whole. However, the following exchange makes explicit an interesting anecdotal statistic.

Scott: What is the likelihood that, as a frontline supervisor, I get from 4 to 12 ? What is your percentage if you had a $1 \%$ to $100 \%$ chance? - return to the theory of action and recall that it is at number 4 that the user interfaces with the development process and at number 12 that she is in a continual process of development and growth.

Oprah: Greater than one and less than 100. (laughter)

Lynn: So, what that's really saying is, 'What are the chances that you are going to have some focused development discussion and planning with your supervisor, that you are going to have the opportunity to be coached, and be engaged in some development activities and that, in fact, your KSAs will improve?'

Scott: That the business is going to pick a model that it is going to be implemented, that I am going to be participating in those resources and tools and that I am going to be continuously growing as a leader... with these activities in mind...

Lynn: Um...Okay 
Oprah: I am just waiting. I am going to see if you are more or less optimistic than I...

Lynn: In 2006, the new year that we are going into...

Oprah: And we will never know because we will not measure it. I think 50 percent is very unrealistically generous.

Lynn: I was going to say probably realistically 40 percent.

To be fair, later in the meeting Lynn said that she thought it may be above 50 percent. Regardless, it is difficult to know what the effect of the FLES has been on the organization; with the elimination of Lynn's function, it may never be known. Once again, the individual businesses are responsible for determining measurement, tracking and initiative evaluation.

All in all, a close similarity between the literature and what is happening at Beta Company does not exist. First, the literature exists in a vacuum. For instance, the literature has the ability to exist in an ideal scenario with plenty of money, time and human resources to work on initiatives. Aligning the performance appraisal system with leadership development sounds simple on paper but, in reality, an individual could spend the entire year working on aligning these two systems. Beta Company experienced this when implementing its 360 Leadership Assessment Process. Oprah spent the vast majority of her time on tasks such as developing the instrument, translating the instrument and testing the instrument. I imagine when practitioners read the literature they immediately see these barriers (real and perceived).

A second observation I made through this process was that the literature does not address two important aspects of leadership development, marketing and implementation. 
In the end, each can make or break the program. An informal observation of mine is that so much time may be spent on developing the "development tools" that the marketing and implementation of the system can be overlooked and neglected. For instance, once each individual business within Beta Company determines how leadership development works within its organization, an essential component is marketing and implementation. I suggest that a number of leadership development initiatives in the business world have not adequately planned for these components of the process. What some do not realize is that OD or leadership development is a field in itself with all its own jargon and idiosyncrasies. To the sales manager, e-learning and a 360 are foreign concepts. I do not know of literature that has specifically discussed how an organization can better market and implement leadership development initiatives.

Another difference between the literature and this organization is intentionality. Having an awareness of the literature allows an individual the luxury of intentionally choosing theories, development tools and evaluation techniques. Organizations in a position to intentionally consider these factors are more likely to choose a theory of action that is realistic.

A final difference between the literature and Beta Company is that it does not clearly define a realistic objective for measurement. A number of authors have written about evaluating leadership and training, and development; returning to Conger's suggestion, "Most would agree that to seriously train individuals in the arts of leadership takes enormous time and resources - perhaps more than societies or organizations possess, and certainly more than they are willing to expend" (p. 38-39). As Oprah and others embark on the journey of "creating leaders" at Beta Company, is it far too 
unrealistic? Given organizational constraints, human resources constraints, time and finances, what can Ms. Reeves and her team realistically hope to accomplish? This is a big question because, by their own account, the organization has a theory of action in place that may work only 40 percent of the time. However, the literature does not address this issue on a large scale. A rosy picture of developing a leader is painted without "real life" discussions about what it is like to be in the trenches and back alleyways of leadership development; a place where one does not know if it is affecting the bottom line. So what is realistic? What is a realistic theory of action for leadership development? Is the literature setting up organizations for failure?

\section{Chapter Four Summary}

Chapter Four contained four primary sections. First, I discussed the specific case and outlined several aspects of the organization and its Frontline Leadership Excellence System. In the second section, I outlined the theory of action and provided commentary on the components therein. The third section provided commentary on observations and findings of the debriefing meeting. In this section, I identified potential areas of focus for Chapter Five. The chapter concluded with a comparison of the leadership development literature with the Beta Company's FLES.

Chapter Five focuses on identified gaps in the literature, thoughts on the theory of action process and identifies suggestions for practice. Likewise, I revisit some of the reactions from Chapter Four and discuss their implications for future research and practice. 


\section{IMPLICATIONS: CHAPTER FIVE}

The purpose of this study was to examine how academic notions of leadership development compare and contrast with the theory of action that guides corporate leadership development initiatives. A secondary purpose was to analyze the process and potential extensions of the user-focused theory of action approach. Chapter One introduced the background, purpose and problem statement. Chapter Two served as a general overview of the leadership development literature and began with a broad overview of the landscape and narrowed to focus on five specific areas of leadership development: leadership theory, organizational context, adult development and learning, development tools, and evaluation. Chapter Three began with a discussion situating me in the research and making transparent the political, social and cultural biases I potentially brought to the study. I then discussed case study methodology and its inherent benefits and drawbacks. Case study methodology served as a container for the study and the userfocused theory of action approach served as a technique for data collection. I discussed this approach and then shared the framework and results of the pilot study and the overall study design. Chapter Three concluded with a discussion of methods of addressing validity, reliability and ethical considerations inherent in research. Chapter Four focused

on the actual study and the findings. I discussed the specific case examined and explained the organization's theory of action and the validity assumptions. I concluded with a comparative benchmark of Beta Company's approach with that of the literature on leadership development. 
In part, this has been a study about academic and practitioner-based notions of leadership development. Why do practitioners think what they are doing will yield certain results? How does the literature address these notions? How do they compare and contrast?

This study has offered a glimpse into these two parallel, yet different worlds. This chapter contains three sections. The first section is an examination of potential gaps within the leadership development literature. The second focuses on the user-focused theory of action process and offers suggestions for practice. The third section focuses on extensions of the user-focused theory of action approach.

\section{The Literature on Leadership Development}

As highlighted in Chapter Two, a number of authors have written about leadership development. A primary gap, however, has been the literature's apparent incoherence. For instance, the theories, models and definitions of leadership development are difficult to locate. However, a background rooted in the literature in conjunction with my experience at Beta Company helped me identify two areas for further investigation.

One is the marketing of leadership development. By nature, those who create leadership development initiatives are educators/trainers, curriculum designers and builders of learning opportunities. However, leadership development is about asking people to change behavior. Heifetz \& Linsky (2002) suggest that “To persuade people to give up the love they know for a love they've never experienced means convincing them to take a leap of faith in themselves and in life" (p. 26). As a result, leadership development is just as much about individual change, organizational change and behavior modification as it 
is anything else. Therefore, inherent in a leadership development endeavor is the need to convey why these changes in behavior benefit participants and the organization. In a sense, it is "conceptual sales." The second gap is the implementation phase of a leadership development initiative. Practitioners and scholars should align and integrate leadership development initiatives with the culture of the organization. A realistic implementation plan yields a more realistic theory of action which, in turn, causes better results.

\section{Marketing/communication of leadership development initiatives}

If the end users and their supervisors are not aware of how the initiative or system can benefit them, it is likely that resource utilization will be low or misguided. The creation of a clear and simple marketing plan helps practitioners convey the benefits of participation in the development activities. How will it help the participants in their careers? How will it make the supervisor's job less of a challenge? The focus should be on gaining commitment from constituents, not simply compliance. In subsequent conversations with "Oprah," we discussed the need to communicate the 360 process effectively. After all, it has been "off-line" for more than a year and there are a number of new supervisors in the organization. Naturally, communication can be a challenge at a number of levels in a global organization.

The leadership development literature does not provide guidance on this topic. I am confident that such information would be of interest to organizations of all sizes. Similar to the implementation phase, I assert that some of the aforementioned executive's "missing 50 cents:" "Probably at least half of every training dollar we spend is wasted - 
we just don't know which half" - can be found in marketing and communication. Some key questions that could serve as guideposts in this phase include:

- Who needs to understand the leadership development initiative?

- How is the initiative being communicated to key constituents?

- How do initiative architects plan to gain commitment vs. compliance?

- How will the initiative benefit the end user? Why should they invest their time?

- What's in it for the manager of the supervisor? Why will the initiative benefit them?

- How does everything work? What is the process? Is it explained in a simple and straightforward manner? Is it user friendly?

- How does the implementation plan interface with the marketing and communication plan?

- How will feedback be gathered from the end users and his supervisors?

Marketing and communication of leadership development initiatives should be a major focus. A lack of focus in this area may serve as a stumbling block for organizations as they implement leadership development initiatives.

\section{Implementation of leadership development}

In a conversation with Oprah following the formal research process, she asked, “Are we doing the wrong things?" My response was simple, “I don't think you are necessarily doing the wrong things, but the things you are doing are having a difficult time getting to the end user." There are simply too many barriers for the current theory of action to work as designed. In my mind, another portion of the "50 cents" discussed in Chapter One can be found in the implementation process. Returning to the comments 
made by Oprah and "Lynn" in the debriefing meeting, less than 50 percent of their frontline leaders are experiencing the FLES in the intended way. Leadership development architects build the 360 , the personal development plan process, and have the ability to pick and choose e-learning courses and products. However, that is only one part of a larger equation; if the end user is not using these materials and the organization does not foster a culture of growth and development, there is a missed opportunity. Returning to McCauley, Moxley, \& Van Velsor (1998):

Other organizational systems must support the leadership development process. To be fully effective, a development system must be integrated with the organization's other processes: management planning, performance management, job selection, reward and recognition systems and even mistake systems. The confluence of these processes determines the relative effectiveness of any one development activity. (p. 228-229)

The complexity of this phase is infinite. Myriad variables exist and there is no quick fix. That said, the development of guideposts for implementation will help organizations better translate their theory of action into reality; therefore, reducing validity assumptions and better serving the end user and the organization. Although a heading in the model for "implementation" currently exists, others are appropriate. For instance, one might describe how the architects build a coalition of "partners" to assist in making the initiative a reality for end users. Bass (1990) asserts that "most important to whether training will modify behavior back on the job is the trainee's immediate supervisor" (p. 854). 
Managing the implementation phase of leadership development is a foundational piece of the leadership development puzzle. Key questions that may assist the practitioner in implementation include:

- How does the initiative link with the strategic direction of the organization?

- Who are the primary stakeholders and does programmatic success assist in meeting their goals?

- How will the initiative be communicated? All at once? In pieces? Is it voluntary? Mandatory?

- How will the initiative be marketed to the end users and those in their sphere of influence?

- Upon whom does the implementation depend? How are individuals made aware of their roles, expectations and objectives?

\section{The User-Focused Theory of Action Process}

The process of making explicit Beta Company's theory of action was quite enjoyable. It began with a set of interviews with Lynn and Oprah. These interviews varied in length (usually about an hour) and started with my attempt to understand the organization and the Frontline Leadership Excellence System. I have identified seven hallmarks that assisted in the process of making explicit Beta Company's theory of action: (1) organizational awareness and understanding; (2) a semi-structured interview process; (3) adaptation of Patton's original process; (4) a symbiotic process of theory development; (5) a knowledge of the leadership literature; (6) time; and (7) willing/open participants. 


\section{Organizational awareness}

A strong grasp of the organization and historical information was helpful in laying the foundation for the theory of action. As Oprah and Lynn described, they have witnessed different swings of the pendulum. Each has seen intense periods of training and development with a centralized approach. Now the organization is moving toward a more decentralized approach to leadership development. All of this information was valuable when working to understand the context of the organization's theory of action. Perhaps most importantly, it helped me understand that the organization's theory of action is not necessarily what they (Lynn, "Ms. Reeves" and Oprah) would like it to be. However, given a messy and "real life" set of parameters, they have done the best they can to develop programming and resources that develops the end user. This sentiment hit home when Lynn said:

It's lack of funds - budget. It's been really, really frustrating. We've had to do all this stuff on a shoestring. And I understand why, and there's good reason for it. If we had a little bit of money to spend, I think we could beef up what we're doing across all areas. We could offer more. Right now, we have limited resources 'people resources' and 'dollar resources.' I'd like to see us do more of what we're doing. Additional funding will help us do that. Leadership development is tough it's hard, it's very hard.

Understanding this helped me better understand what the organization was trying to accomplish given its spending constraints. It helped me understand their course of action and made clear a discrepancy between the literature and practice - practice is not as neat and clean as it is in the literature. Returning to Chapter Two, the suggestion that 
leadership development initiatives link to organizational systems is easy to say on paper, but difficult to create in a global organization.

\section{Semi-structured interview process}

The interviews process was semi-structured. For the most part, I did not enter the meetings with a prescribed set of questions; and when I did, questions changed in an effort to make the process seem more organic and relevant given the conversation. This is not to say that I left meetings with unanswered questions. I did not. I did, however, hold off on certain questions when appropriate and waited to see if participants mentioned issues such as implementation, evaluation and marketing. That said, a few direct questions were helpful in making explicit the organization's theory of action. For instance, when asked, "Ideally how would it (FLES) affect participants?" Lynn responded with a concise description of "how FLES is supposed to work" when she replied:

Well, if they're doing what they should be doing, the frontline supervisor would understand what the expectations are. In addition, they would know the leadership imperatives and what they mean to them - in terms of their behavior and their actions. They (frontline supervisors) would be participating in the 360 process, so they'd be getting feedback on their behaviors, their actions, and how those expectations are being perceived by their managers, their peers, and their employees. This feedback would help them prepare for their employee development planning discussion. Ideally, the process would impact their whole job.

Another question that helped me better understand the ultimate objectives (effect on the organization) was, "If you were to have to come up with the ultimate objectives of the FLES, what would they be?" To this question, Lynn responded: 
I'd say it's to perpetuate great leaders who can help the company be successful; and that means adjusting leadership at every level. Everything that we are doing surrounds creating, sustaining and improving leadership effectiveness, because it is critical to a company's success. Without it we won't survive.

Oprah expressed similar sentiments when she said, "To have competent leaders...more than competent, but you know...very competent; excellent leaders capable of doing their job."

\section{Adaptation of Patton's original process}

To better understand the organization's theory of action, I had to break from what Patton (1997) had originally outlined as the process and major sub-headings. To me, it made more sense to outline the process in the following manner.

- Program Development and Background Information - This information is the historical information and underpinnings upon which the initiative exists. In ways, it is the foundation. Within this section, I housed the curriculum, development tools, time commitment, resources and process. A number of validity assumptions exist in this phase. For instance, the Frontline Leadership Excellence System exists upon the notion that the organization's 2005 Leadership Imperatives do, in fact, drive leadership excellence. If this is not true, then the initiative may be in trouble from the beginning.

- Immediate Objectives (Program Implementation) - I defined Immediate Objectives as the "program implementation" phase of the FLES. After the content, curriculum and development tools are created, the question becomes "How do we make this 'live' in the organization? How will it reach the 5,000 
frontline supervisors intact, and as we designed?" Again, this phase contains a number of key validity assumptions that will likely hinder progress if not addressed. For instance, the current theory of action assumes that the regions and businesses have clearly defined roles as the organization moves to this new model. Given the fact that each individual business is developing its own theory, I wonder who has decision making authority regarding roles. How will it all be managed?

- Intermediate Objectives (Individual Effect) - This phase of development focuses on the development of the individual. Foundational elements have been delivered and are now interfacing with the end user. What the end user experiences (in this case a frontline supervisor), is another crucial phase in the process. After all, a primary assumption upon which the initiative exists is that developing leadership capacity in individuals will foster organizational development and business results. An additional assumption at this phase is that managers of the supervisors are, in fact, helping the initiative "live" within the organization.

- Ultimate Objectives (Organizational Effect) - Ultimately, Beta Company's goal is that leadership development will result in business success. However, organizational effect is contingent upon what has been occurring at lower levels of the causal chain. Thus, it is unrealistic to think that the overall theory of action can work unless all levels (or many) are working as they should. 
These were the four primary sub-headings for the current study. However, upon reflection, I envision others such as marketing/communication, linkage to culture and evaluation. These additions may help practitioners and researchers focus on crucial components of the leadership development process. To present it in the form of an equation:

Clear objectives + sound development tools/learning activities + resources + effective implementation + effective marketing/communication + linkage to HR systems + transfer of training techniques + evaluation $=$ effective leadership development.

Of course, other variables exist. For instance, The Center for Creative Leadership (2005) asserts that the individual's ability to learn is an additional variable. However, the above components are some of the major guideposts that could serve as headers for the user-focused theory of action approach.

\section{A symbiotic process of theory development}

The progression of the theory of action yielded seven versions and it has been a symbiotic process between researcher and participants. After two meetings with Oprah and Lynn, I began formulating the theory of action and brainstorming potential validity assumptions. Meeting three with Oprah and Lynn provided an opportunity to explain the "draft" theory of action and served as a venue to receive feedback about content. In some ways, this meeting was a challenge because it was content heavy. My impression was that participants needed time to "digest" the four page theory of action, so I asked each to send me feedback, suggestions, additions or deletions. Both did. After I gained agreement 
between Oprah and Lynn, I presented the information to Ms. Reeves over the phone. I spent 15 minutes with her discussing the informed consent form, the general purpose of the study, as well as the draft theory of action so she had a level of understanding and agreement prior to the debriefing meeting with all three participants. A hallmark of this process, however, is that all parties come to agreement with the final theory of action which provides the researcher or consultant a sound point of departure. Reaching a common theory of action was not a challenge in this instance; however, I imagine it could be in certain situations.

\section{Participant ownership}

Participant ownership is a fundamental cornerstone of this model and affords the consultant or researcher the luxury of saying, "This is your theory of action" as the group begins deconstructing or taking a closer look at assumptions. Furthermore, working with participants affords the opportunity to establish rapport, build relationships and better understand their perceptions regarding the organization and the leadership development initiative. There is an inherent "give and take" throughout the process and, through the semi-structured interview process, all participants have some influence over theory development. There is power in the participants "owning" their theory and, although I helped along the way, it was created as a group.

\section{Knowledge of leadership literature}

I found it helpful to be familiar with the leadership literature. This foundation not only helped me quickly understand the programs and processes at Beta Company, but 
also gave me a unique lens from which to view the organization. Moreover, as we discussed validity assumptions, I had the ability to draw upon knowledge of leadership theory, transfer of training, linkage to human resources systems, adult learning, adult development, and the evaluation of leadership development.

However, knowledge of the literature alone does little to help an organization with real world constraints and barriers, especially when it comes to implementation, marketing, transfer of training and working through "real world" barriers. The participants each have worked in learning and development for more than 20 years and still struggle with the correct course of action.

\section{Time}

The luxury of time was a benefit to me as a researcher. In the pilot study, I did not allow enough time for the process which created a stressful experience. The process of working with Beta Company unfolded over two months, which allowed me time to meet with participants and reflect on the meetings, the transcripts and the organizational documents. I would not need this amount of time in the future, but it was a primary benefit in this case. The researcher should set aside ample time for the process to unfold; especially when new to the process.

\section{Willing and open participants}

As mentioned by Patton (1997), one of the potential drawbacks of this process is that, as practitioners struggle to share their theory of action, they may become defensive or frustrated by the process. In this instance, the initiative $(F L E S)$ is the direct product of 
those with whom I was working. As a result, the process could have made participants feel defensive and uncomfortable because the primary purpose of the activity is to unearth their theory of action and identify validity assumptions to locate and address faulty logic. In my experience at Beta Company, I tried to minimize this dynamic and expressed to participants that organizational barriers and factors outside their control have a major effect on the FLES. In addition, I expressed that this is not an activity intended to shine a light on them, it is an activity to shine a light on organizational barriers and decisions that may hinder the program's ability to succeed or have the intended effect on participants. That said, I found the participants at Beta Company to be warm, open and more than willing to share their feelings throughout the process.

\section{The User-Focused Theory of Action - Extensions for Use}

The user-focused theory of action approach has potential for the field of leadership development. Naturally, areas for further investigation exist. The following section highlights a number of extensions, thoughts and observations. These are rooted in the debriefing session with study participants and my own reflections. These extensions include using the approach:

- in developing a realistic theory of action;

- as a planning tool;

- as a diagnostic tool;

- as a springboard for brainstorming;

- as the groundwork for formal examination of the literature;

- outside of leadership development;

- in the development of directions and parameters for use;

- in communicating the technique; 
- in aligning with Appreciative Inquiry (AI); and

- in making explicit the theory-in-use.

First, the process may help organizations create a more realistic theory of action. Helping an organization develop a more realistic theory of action will not only increase the credibility of practitioners, but also it will help them affect business results. Second, the process may help in the planning stages of a leadership development initiative. Using the user-focused theory of action approach as a lens from which to view the planning process may be an important resource. Third, for those organizations already in the midst of a leadership development initiative, user-focused theory of action approach may serve as a diagnostic intervention that can help practitioners intervene and adjust as needed. A fourth area for investigation is the tool's ability to serve as a springboard for brainstorming. A fifth extension is located in the leadership development literature. Once the theory of action and validity assumptions are explicit, a natural "next step" is to look to the literature for suggestions. A sixth extension is the user-focused theory of action approach's use outside of leadership development. I suggest that this approach translates into other fields where training and development occurs and/or a change in behavior is the goal. A seventh extension is the development of more formalized directions and parameters for use; a number of these were identified in the previous section (Thoughts on the User-Focused Theory of Action Process) and were discussed. Eighth, the technique should be communicated in a simple and straightforward manner. As I have explained my research to friends and family, I have continually struggled to discuss the user-focused theory of action approach. There must be a better way to communicate this technique so others can understand the power and benefit of the process. A ninth 
extension is combining the current approach with an Appreciative Inquiry. A final extension relates to Argyris and Schön's (1978) original thinking about espoused theories and theories-in-use. After the theory of action is made explicit, a discussion with end users would likely yield the theory-in-use and additional areas for exploration.

\section{Developing a realistic theory of action}

Perhaps the greatest value of this exercise is the opportunity to view the theory of action and validity assumptions in their entirety. This tool provides a unique lens that, when examined, has the power to pinpoint areas of weakness and gaps in logic. Returning to Lynn and Oprah's assertion that less than 50 percent of Beta Company employees were experiencing the model as described, a natural extension of this tool is to develop a more realistic theory of action. Doing so benefits the organization in a number of ways. First, it helps the organization create an initiative that will be more likely to have the desired effect on the end user. Second, it may help initiative architects place boundaries on their process because developing leadership capacity is a lifelong endeavor. To think that an organizational initiative will foster results of this nature may be outside the span of their control. Placing boundaries around the theory may foster a more realistic opportunity to develop the end user. I assert that Beta Company's current theory of action has a low chance of affecting the end user in the manner described. A number of barriers exist for the FLES to work as the theory of action describes. So what needs to change? How could the theory of action be made more realistic while still supporting the organization and its business goals? One example may be that, in conjunction with the development plan, frontline supervisors work on a project (within their span of control) 
that yields a financial benefit to the organization. As part of the process, the supervisor could reflect and discuss the project with his manager. This is more realistic than having leaders "poached" as an ultimate objective (see number 13 in the theory of action). Moreover, the project described can be tracked, quantified and directly attributed to the FLES.

As a planning tool

My primary goals for this dissertation were to outline the theory of action, determine the validity assumptions and benchmark this information with the leadership literature. This was accomplished. However, from Lynn's perspective, as expressed in the debriefing meeting, the process only brought the group to a certain place - a place that begged the question, "Is that it?" or "Now what?" When participants were asked to brainstorm "next steps" for such a tool, they were short on ideas. However, it is important to note Lynn's initial reaction, "You know what I would say, that quite honestly, that this does not spark any additional insights or thoughts" is important because it is the springboard for the "so what, now what" discussion. To describe it another way, "Great, now all this is in front of us, where do we go from here? How do we make it better? How do we tighten up the process? Which assumptions can we manage? Which assumptions are outside our control?"

Using the process as designed (with slight alterations) could serve as a planning tool for an organization in the initial stages of developing its initiative. Such a process may look something like this:

- Investigate organizational context 
- Determine primary sub-headings. As discussed previously in this chapter (Adaptation of Patton's Original Process) these may include: desired effect on the organization, desired effect on the individual, implementation, marketing, development tools/learning activities, identification of organizational resources, marketing/communication, linkage to HR systems, transfer of training techniques and evaluation.

- Once these sub-headings exist, a brainstorming session occurs to capture the team's thoughts and ideas for each of the major sub-headings.

- Organizational constraints or limitations should be discussed and help initiative architects identify realistic components of the initiative.

- Once these have been captured, the committee should begin constructing the theory of action or mapping each step in the process. Doing so will produce a logical progression of how the initiative is supposed to work.

- Once the theory of action is developed, participants should brainstorm validity assumptions. Once these assumptions are identified, they should be managed or simply accepted as items that cannot be controlled. These should be flagged and revisited throughout the process.

- Next, a focus group may help initiative architects identify and determine additional thoughts for the committee.

- The final step is to begin work on the initiative along with a continual performance improvement process.

A variation of the above mentioned method exists. In her book Planning Program for Adult Learners: A Practical Guide for educators, trainers and staff developers, Rosemary Caffarella (2002) outlined a checklist for planning programs. She has several section headings which include: discerning the context, building a solid base of support, identifying program ideas, and developing program objectives. Under each of these headings she outlines a number of "things to think about." For instance, under Devising Transfer-of-Learning Plans, she suggests: 
- Be knowledgeable about the major factors that influence transfer of training.

- Choose transfer strategies that are most useful in assisting participants to apply what they have learned.

- Decide what transfer of training strategies should be employed before, during and/or after a program (p. 24).

A similar checklist exists for leadership development. After using the userfocused theory of action approach in several organizations, I am confident that themes and patterns will emerge. As a result, a planning resource similar to Caffarella's could be developed.

As a diagnostic tool

In a similar vein, the technique may serve as a diagnostic tool. For organizations already in the midst of a leadership development initiative, the technique is an ideal activity to pause and evaluate the program. After all, the approach helped Lynn and Oprah "ballpark" the effectiveness of the FLES.

When used as a diagnostic tool, altering the user-focused theory of action approach is a necessity. The process may look something like this:

- Make explicit objectives of the exercise. Are the objectives to develop a more realistic theory of action? Investigate the current model for potential gaps? This should be clear from the outset.

- Investigate organizational context and meet with initiative architects or those in decision making roles to make explicit their current theory of action. It is important to allow the initiative architects the opportunity to share everything they can prior to a more specific investigation of "missing" pieces of the 
theory (e.g., perhaps they have not fully examined their process for marketing).

- Based on organizational context and needs, determine primary sub-headings for investigation. These may include: desired effect on the organization, desired effect on the individual, implementation, marketing, development tools/learning activities, identification of organizational resources, marketing/communication, linkage to HR systems, transfer of training techniques and evaluation.

- After making explicit the theory of action, examine validity assumptions with participants and gain agreement.

- Next, revisit objectives for the exercise and, if appropriate, pinpoint five areas for further investigation. These may be current aspects of the theory of action or areas not yet addressed by initiative architects. For example, if marketing has been non-existent, it may become an area for further investigation (AFFI).

- Once the AFFIs exist, participants should brainstorm possible solutions and discuss appropriate organizational constraints or limitations.

- Five realistic initiative alterations are appropriate here. These should be within the committee's span of control and implemented with relative ease.

- Ideally, this is an iterative process similar to any performance improvement or quality methodology (e.g., PDSA - plan, do, study, act).

An additional diagnostic extension could be an inventory for organizations in the midst of a leadership development initiative. Similar to the "planning" suggestions discussed earlier, common themes and problems will be identified as the user-focused theory of action approach gains in use. By capturing these common barriers and naming them, leadership development architects have the opportunity to self-assess. The instrument may be known as the Organization Leadership Development Inventory (OLDI) and could ask initiative architects to answer statements such as: 
- I feel the leadership development initiative is working as it should.

- Ultimately, we would like to have the following effect on individuals.

- Ultimately, we would like to have the following effect on the organization.

- Managers are supportive of leadership development initiatives.

- The leadership development initiative links to HR systems.

- The leadership development initiative is rooted in leadership theory.

- The leadership development initiative has clear objectives.

- The foundation of the leadership development initiative relies on a realistic theory of action.

- The leadership development initiative has the support of key decision makers.

- Development tools link to the performance management process.

- Developmental activities and assignments link to financial return on investment.

A natural challenge to this approach is that it is a stock instrument that does not adequately represent organizational context and, in fact, may negate the need for the user-focused theory of action approach. However, I envision an instrument such as this could change and adapt the line of questioning similar to an "in person" researcher. The technology would investigate organizational context and all other aspects of the leadership development initiative. Although conducted in a different format, such a tool could assist organizations in making sense of their theory of action and address validity assumptions accordingly. In addition, an instrument of this nature could assist the researcher to more quickly understand the organizational context and programming prior to direct one-on-one work. 


\section{A springboard for brainstorming}

I suggested in Chapter Four that informal brainstorming began in the debriefing meeting. This was a natural outgrowth of the process and could be formally built into it. For instance, once the theory of action is explicit, one could pinpoint key validity assumptions for management and, if possible, elimination. In so doing, the group could benefit from formalized brainstorming which is simply a process of building upon one another's ideas. Doing so may help architects manage and control or revise key assumptions.

\section{A formal examination of the literature}

The literature holds important information and, although it is not a panacea, may offer important clues. For instance, in their meta-analysis, Kluger \& DeNisi (1996) found that upon receipt of feedback; (1) one third of participants improve; (2) one-third maintains the status quo; and (3) one-third decreases in performance. This is an important study to be aware of as an organization embarks on a feedback process. Awareness in this instance may help initiative architects avoid a number of barriers and pitfalls inherent in the feedback process.

In addition, awareness of the leadership development literature brings a level of intentionality to the process. Development tools selected (in part) because of a supportive literature base rather than simple familiarity is a better decision making process. Moreover, the literature is rich in the benefits, challenges and lessons learned - many of which are mentioned in the section on development tools. 


\section{Use outside of leadership development}

This approach finds its roots in the context of "evaluation research." In fact, after the theory of action and validity assumptions, Patton (1997) suggests pinpointing areas for evaluation. However, this approach has clear implications for almost any initiative within an organization. For instance, if a healthcare organization were working to implement the National Patient Safety Goals (set of safety guidelines set forth by regulatory bodies) into its culture, the user-focused theory of action approach could help architects manage and plan for this change in culture. In addition, the approach allows for contextual variables and nuances.

As mentioned, there are technological extensions as well. For instance, an online tutorial or program could walk users through the process under each of the key subheadings. This would not have the same effect as working with people face to face, but it could help architects begin thinking and designing initiatives within the context of this framework. A computer program could even estimate the effect of assumptions. What if Beta Company went into the FLES knowing that there was a less than 50 percent chance of organizational effect? I imagine a different theory would exist.

\section{Directions and parameters for use}

One clear need for an instrument of this nature is directions and parameters for use. Some of the suggestions within this section will sound familiar, and others are new concepts. However, it is important to "house" these in one place. The suggestions for practice are important to the success of this technique. 
According to Patton (1997), a researcher using this method must do at least five things:

1. Make the process of theory articulation understandable.

2. Help participants be comfortable with the process intellectually and emotionally.

3. Provide direction for how to articulate espoused theories that participants believe undergird their actions.

4. Facilitate a commitment to test espoused theories in the awareness that actual theories-in-use, as they emerge, may be substantially different from espoused theories.

5. Keep the focus on this to make the evaluation useful (p. 223).

I offer a few other suggestions because the above mentioned guidelines focus only on the process of making explicit the theory of action in an evaluation setting. In the context of using this instrument for a leadership development initiative, I would add the following general suggestions for practice.

1. Gain an understanding of organizational context and identify research participants.

2. Define objectives and desired outcomes for the user-focused theory of action approach.

3. Make the process of theory articulation understandable.

4. Help participants be comfortable with the process intellectually and emotionally.

5. Provide direction for how to articulate espoused theories that participants believe undergird their actions.

6. Be clear about the major sub-headings for each step of the causal chain and identify additional sub-headings specific to the organizational context.

7. Work with participants to make explicit the theory of action and validity assumptions. 
8. Identify areas of focus. Pinpoint key validity assumptions that may hinder progress if not addressed.

9. Discuss organizational constraints and brainstorm possible solutions to the identified assumptions and revisit objectives.

10. Evaluate the process with participants.

\section{Communicating the technique}

In casual conversation with friends, family and even those familiar with the literature, I struggle to explain this approach in a simple and straightforward manner. In my experience, the term user-focused theory of action approach sounds highly academic and obtuse. Other terminology such as "theory of action" and "validity assumptions" are confusing and could be better expressed. There has to be a better way to communicate what this approach, technique and tool provides. Although I do not yet have a concrete solution, the following section briefly explores possibilities.

As I explained the process to Lynn, she mentioned the terminology "key success factors" in place of "validity assumptions." The term "key success factors" is easy to understand and one can quickly visualize the meaning of this phrase. In the future, I would like to partner with practitioners to create terminology that is more accessible and easy to understand. Some initial thoughts include:

- Theory of action - logic roadmap, causal chain, chart, plan, logic trail, logical model, ideal model or process

- Sub-headings - guideposts, checkpoints or imperatives

- Validity assumptions - key success factors, areas for further investigation, road blocks, imperatives or essential elements 


\section{Alignment with Appreciative Inquiry}

At first glance, the user-focused theory of action approach has little in common with Appreciative Inquiry (AI). According to Cooperrider \& Whitney (n.d.), "Appreciative Inquiry is about the coevolutionary search for the best in people, their organizations, and the relevant world around them. In its broadest focus, it involves systematic discovery of what gives "life" to a living system when it is most alive, most effective, and most constructively capable in economic, ecological, and human terms" (p. 3). A natural link between AI and user-focused theory of action approach exists. At first glance, this may not seem the case because user-focused theory of action approach focuses on gaps in logic and assumptions. I suggest that AI is the opposite of validity assumptions. Rather than the "gaps," it focuses on the "energy" or "commonalities" that provide energy and life to an initiative. An extension of the current process could be the addition of a third column. For example:

\begin{tabular}{|l|l|l|}
\hline Appreciative Inquiry & Theory of Action & Validity Assumptions \\
\hline
\end{tabular}

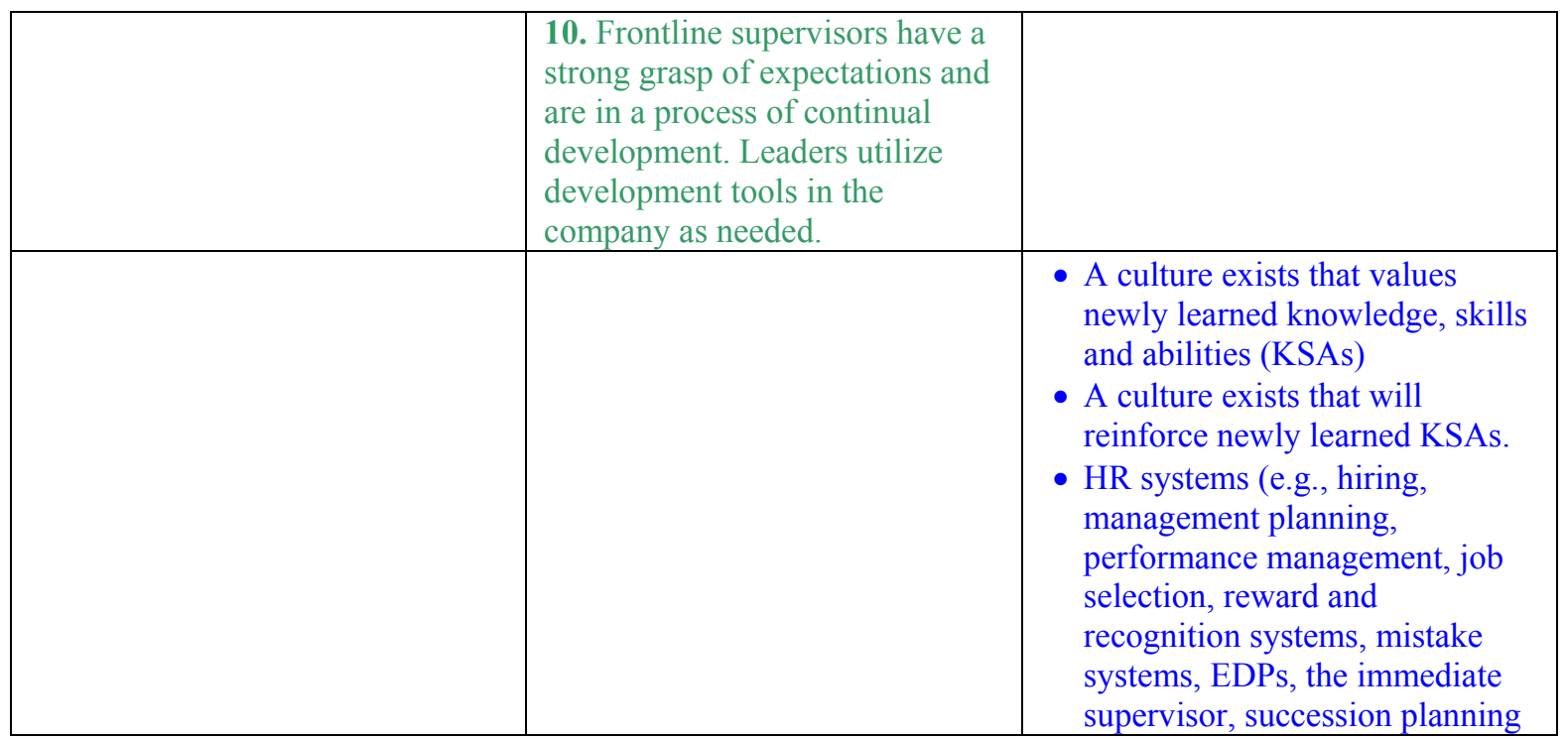




\begin{tabular}{|l|l|l|}
\hline & & $\begin{array}{l}\text { and career development) align } \\
\text { with the development } \\
\text { programming. }\end{array}$ \\
\hline & $\begin{array}{l}\text { 9. Frontline supervisors improve } \\
\text { in their knowledge, skills and } \\
\text { abilities (leadership capacity). }\end{array}$ & \\
\hline
\end{tabular}

In the above model, the second column (theory of action) and the third column (validity assumptions) remain the same. However, to the left of the theory of action column could be the possibilities, areas of strength and where the energy lies. Returning to Cooperrider \& Whitney (n.d.):

AI involves, in a central way, the art and practice of asking questions that strengthen a system's capacity to apprehend, anticipate, and heighten positive potential. It centrally involves the mobilization of inquiry through the crafting of the 'unconditional positive question' often-involving hundreds or sometimes thousands of people. In AI the arduous task of intervention gives way to the speed of imagination and innovation; instead of negation, criticism, and spiraling diagnosis, there is discovery, dream, and design. AI seeks, fundamentally, to build a constructive union between a whole people and the massive entirety of what people talk about as past and present capacities: achievements, assets, unexplored potentials, innovations, strengths, elevated thoughts, opportunities, benchmarks, high point moments, lived values, traditions, strategic competencies, stories, expressions of wisdom, insights into the deeper corporate spirit or soul -- and visions of valued and possible futures. (p. 3)

Throughout the theory of action approach, opportunity for the above exists. Whether the instrument serves as a planning tool or diagnostic instrument, the AI concepts align well. As a result, potential gaps and opportunities are named, and acted upon. The 4 D Cycle (discovery, dream, design, and destiny) could serve as a framework to fill in the left hand column and capture energy specific to the context. 
Making explicit the theory-in-use

Once the theory of action has been made explicit, the investigator has a number of other levels to evaluate. The theory of action describes how initiative architects intend the model to work; also called the espoused theory. Returning to Argyris (1997):

Human beings hold two different master designs. The first incorporates the theories humans espouse about dealing effectively with others. The second design involves the theories of action they use (i.e., their theories-in-use). Whenever any issue is dealt with that activates embarrassment or threat, we have found a systemic discrepancy between the espoused theories and the theories-in-use and a systemic unawareness of the discrepancy while individuals are producing it. (p. 10)

An investigation of the theory-in-use would yield interesting results. For instance, how does the average supervisor of an individual participating in FLES perceive the process? What perceived benefits do they see? Which aspects have they incorporated? Which aspects do they deem inappropriate or not needed? Understanding how they perceive the FLES works would provide Oprah, Lynn and Ms. Reeves with valuable information as they choose future courses. A random sample of supervisors and their managers would yield data rich in information that may help initiative architects determine the level to which the program is affecting the end user as it is designed. Such an investigation would also yield additional assumptions that Lynn, Oprah and Ms. Reeves may have never thought of; therefore, a more realistic picture of the system as it actually exists in the organization. This could be shared as a visual: 


\begin{tabular}{|l|l|l|}
\hline Theory-in-Use & Theory of Action & Validity Assumptions \\
\hline
\end{tabular}

\begin{tabular}{|c|c|c|}
\hline $\begin{array}{l}\text { Depends on the supervisors } \\
\text { and how long they have } \\
\text { been with the organization. } \\
\text { Most learning occurs on the } \\
\text { job. }\end{array}$ & $\begin{array}{l}\text { 10. Frontline supervisors have a } \\
\text { strong grasp of expectations and } \\
\text { are in a process of continual } \\
\text { development. Leaders utilize } \\
\text { development tools in the } \\
\text { company as needed. }\end{array}$ & \\
\hline $\begin{array}{l}\text { - Yes } \\
\text { No } \\
\text { - No- with the exception of } \\
\text { EDPs. }\end{array}$ & & $\begin{array}{l}\text { - A culture exists that values } \\
\text { newly learned knowledge, skills } \\
\text { and abilities (KSAs). } \\
\text { - A culture exists that will } \\
\text { reinforce newly learned KSAs. } \\
\text { - HR systems (e.g., hiring, } \\
\text { management planning, } \\
\text { performance management, job } \\
\text { selection, reward and } \\
\text { recognition systems, mistake } \\
\text { systems, EDPs, the immediate } \\
\text { supervisor, succession planning } \\
\text { and career development) align } \\
\text { with the development } \\
\text { programming. }\end{array}$ \\
\hline $\begin{array}{l}\text { - Supervisor does not agree } \\
\text { that this is occurring. }\end{array}$ & $\begin{array}{l}\text { 9. Frontline supervisors improve } \\
\text { in their knowledge, skills and } \\
\text { abilities (leadership capacity). }\end{array}$ & \\
\hline
\end{tabular}

The theory-in-use column is filled with feedback and comments from the supervisor or manager being interviewed. In the end, the consultant or researcher will have a clear picture of the theory-in-use for this particular employee and his or her unit or department. As more and more supervisors are interviewed, patterns will appear. As with other extensions, technology may serve as a valuable tool. For instance, the theory in action could be transferred to an assessment to which managers respond electronically. Based on responses, concrete numbers and percentages could be attributed to each step of the theory. Likewise, validity assumptions could be confirmed or discounted. 


\section{Conclusion}

Growing up, I spent my summer days with family at a cabin in Northern Minnesota. Blackwater Lake was a respite from the hustle and bustle of everyday life; a place where time stood still and the most important item on the agenda was relaxation. One form of relaxation for my grandmother and family members was assembling puzzles. I remember the boxes piled high, never quite understanding the allure. At the time, Go Fish! and Uno were more exciting. However, there was something about the half-completed puzzles that kept me coming back for a look. At times, it was because I thought I would get lucky and add a piece before jumping in the lake. Other times, it was to check on progress. Day after day, family members would work at it; all with one common goal. As the time at the lake passed, the puzzles would take shape. Eventually, the 1,000 piece puzzle would be completed.

I view leadership development as a puzzle. My hope is that this work adds to the puzzle. Legends such as Bass, Avolio, Day, Conger, Lombardo, Fulmer, Mezirow, Patton, Kegan, Kirkpatrick, Phillips, Zaleznik, McCall, McCauley, Kotter, and Yukl have all sat at the table and worked on the puzzle. Amazingly, individuals in other fields have spent time on their own puzzles and, at times, their pieces somehow fit into ours.

This dissertation has afforded me the opportunity to work on the puzzle of leadership development and has accomplished several objectives. First, I reviewed and made sense of a disparate and segmented base of literature. I aligned themes from leadership theory, adult development and learning, human resources, development tools and evaluation. I then adapted Patton's (1997) user-focused theory of action approach to leadership development initiatives; primarily in an attempt to use it as a springboard to 
examine how academic notions of leadership development compare and contrast with the theory of action that guides corporate leadership development initiatives. At first, it was unknown if the tool could be transferred and utilized in a global organization such as Beta Company. However, with a beneficial pilot study and a few alterations, the approach did transfer. I was able to make explicit the organization's theory of action for the Frontline Leadership Excellence System. The debriefing meeting was a wonderful experience and identified potential challenges (Lynn's "I do not see anything new"), potential extensions (as a planning or diagnostic tool) and general reactions from three individuals whom I respect and admire. A benchmarking of the leadership development literature with the Frontline Leadership Excellence System produced additional learning moments. Viewing the FLES and the leadership development literature through the lens of the user-focused theory of action approach helped me pinpoint additional gaps in the literature (e.g., marketing and implementation) and critical thinking about the process of the approach. These are only a few examples of how the dissertation process in and of itself helped me reflect and make meaning of the experience.

I conducted this research because, at face value, I agreed with the anonymous executive. Returning to his quote:

"Probably at least half of every training dollar we spend is wasted - we just don't know which half" (Martochhio \& Baldwin, 1997, p. 15).

Of course, the quote is vague and a direct correlation between training (which he mentions) and leadership development does not exist. However, I can see how this sentiment is widespread in industry. In fact, a number of studies cited by Ohlott (2005) 
reveal that "a number of research studies conducted at CCL and elsewhere in the 1980s and 1990s support the notion that many managers consider job experiences the primary source of learning" (p. 152). That notion can change.

In my opinion, to get somewhere new, our thinking must change. Apple's marketing slogan captures it best - "Think Different." I am also reminded of the quote by Joel Barker - "What is impossible to do right now, but if you could do it, would fundamentally change your business?" This question takes people to the fringe, which is where a great number of innovations occurs. After all, organizations are spending millions of dollars and often do not have a clear picture of the puzzle and perhaps have not even located all of the pieces (and maybe never will). However, the user-focused theory of action approach is a tool that will help organizations "Think Different." The approach can help organizations better identify where to focus their efforts to better develop individuals, their industry and the world. 


\section{REFERENCES}

Abernathy, D. (1999). Think outside the evaluation box. Training, 53 (2), 19-23.

Alber, A. (1979). Job enrichment for profit. Human Resource Management, 18 (1), 15-25.

American Management Association (1999). 1999 AMA survey on workplace testing. Management Review, 88 (7), 44-47.

American Society for Training and Development. (1990, October). National HRD Executive Survey.

American Society for Training and Development. (1995, August). National HRD Executive Survey.

Argyris, C. (1997). Learning and teaching: A theory of action perspective. Journal of Management Education, 21 (1), 9-26.

Argyris, C. \& Schön, D. (1978). Theory in practice: Increasing professional effectiveness. San Francisco: Jossey-Bass.

Avolio, B. (1999). Full leadership development. Thousand Oaks, CA: Sage.

Avolio, B. (2004). Examining the full range model of leadership: Looking back to transform forward. In Day, D., Zaccaro, S., \& Halpin, S. (Eds.), Leader development for transforming organizations: Growing leaders for tomorrow (pp. 71-98). Mahwah, New Jersey: Lawrence Erlbaum Associates.

Avolio, B. (2005). Leadership development in balance. Mahwah, New Jersey: Lawrence Erlbaum Associates.

Avolio, B. \& Gibbons, T. (1989). Developing transformational leaders: A life span approach. In Conger, J. \& Kanungo, R. (Eds.), Charismatic leadership: The elusive factor in organizational effectiveness (p. 276-308). San Francisco: Jossey-Bass.

Baker, G. (1992). Application report: Instructional design of a computer-assisted work-related literacy program. Journal of Computer-Based Instruction, 19, 33-6.

Bandura, A. (1977). Social learning theory. New York: General Learning Press.

Bass, B. (1985). Leadership and performance beyond expectations. New York: The Free Press. 
Bass, B. (1990). Bass \& Stogdill's handbook of leadership: Theory, research and managerial applications $\left(3^{\text {rd }}\right.$ edition). New York: The Free Press.

Bennett, B. (2003). Job rotation: Its role in promoting learning in organizations. Training Strategies for Tomorrow, 17 (4), 7-9.

Blank, W., Weitzel, J. R., \& Green, S. G. (1990). A test of the situational leadership theory. Personnel Psychology, 43, 579-597.

Bowen, D. D., \& Hall, D. T. (1977). Career planning for employee development: A primer for managers. California Management Review, 20 (000002), 23-35.

Bowsher, J. (1998). Evaluate or else. Training, 35 (12), 88.

Brinkerhoff, R. (1981). Making evaluation more useful. Training \& Development Journal, 35 (12), 66-70.

Brookfield, S. (1986). Understanding and facilitating adult learning. San Francisco: Jossey-Bass.

Brotman, L. E., Liberi, W. P., \& Wasylyshyn, K. M. (1998). Executive coaching: The need for standards of competence. Consulting Psychology Journal: Practice and Research, 50, 40-46.

Brown, K. G. (2001), Using computers to deliver training: Which employees learn and why? Personnel Psychology, 54, 271-96.

Brutus, S., Ruderman, M. N., Ohlott, P. J., \& McCauley, C. D. (2000). Developing from job experiences: The role of organization-based self-esteem. Human Resource Development Quarterly, 11 (4), 367-380.

Burns, J. (1978). Leadership. New York: Harper and Row.

Cacioppe, R. (1998). An integrated model and approach for the design of effective leadership development programs. Leadership and Organization Development Journal, 19 (1), 44-53.

Caffarella, R. S. (2002). Planning program for adult learners: A practical guide for educators, trainers, and staff developers. San Francisco: Jossey-Bass.

Campion, M. A., Cheraskin, L., \& Stevens, M. J. (1994). Career-related antecedents and outcomes of job rotation. Academy of Management Journal, 6 (37), 1518-1542.

Cascio, W. (1991). Costing human resources: The financial impact of behavior in organizations ( $3^{\text {rd }}$ Ed.). Boston, MA: PWS-KENT Publishing. 
Chappelow, C. T. (1998). 360-degree feedback, In McCauley, C. D., Moxley, R., \& Van Velsor, E. (Eds.), The center for creative leadership handbook of leadership development (p. 29-65). San Francisco: Jossey-Bass.

Charan, R., Drotter, S., \& Noel, J. (2001). The leadership pipeline: How to build the leadership-powered company. San Francisco: Jossey-Bass.

Charbonneau, D., Barling, J., \& Kelloway, E. (2001). Transformational leadership and sports performance: The mediating role of intrinsic motivation. Journal of Applied Social Psychology, 31 (7), 1521-1534.

Cheraskin, L., \& Campion, M. A. (1996). Study clarifies job-rotation benefits. Personnel Journal, 75 (11), 31-36.

Clarkson, P., \& Shaw, P. (1992). Human relationships at work in organizations. Management Education and Development, 21 (1), 18-28.

Coleman, E., Patterson, E., Fuller, B., Hester, K., \& Stringer, D. (1995). A metaanalytic examination of leadership style and selected follower outcomes. Unpublished manuscript, University of Alabama.

Conger, J. (1989). The charismatic leader: Behind the mystique of exceptional leadership. San Francisco: Jossey-Bass.

Conger, J. (1992). Learning to lead: The art of transforming managers into leaders. San Francisco: Jossey-Bass.

Conger, J. \& Benjamin, B. (1999). Building leaders: How successful companies develop the next generation. San Francisco: Jossey-Bass.

Cooperrider, D. L., \& Whitney, D. (n.d.). A positive revolution in change: Appreciative inquiry. Retrieved from http://appreciativeinquiry.cwru.edu on December 5, 2005.

Creswell, J. W. (1998). Qualitative inquiry and research design: Choosing among five traditions. Thousand Oaks, CA: Sage.

Davies, J., \& Easterby-Smith, M. (1984). Learning and developing from managerial work experience. Journal of Management Studies, 21 (2), 169-183.

Davis, N. (2001). Building muscle: Developing leaders with follower weight. Organization Development Journal, 19 (3), 27-25.

Day, D. (2001). Leadership development: A review in context. Leadership Quarterly, 11 (4), 581-613. 
Day, D. (2004). Leadership development. In Goethals, G., Sorenson, G., Burns, J. (Eds.), The Encyclopedia of Leadership, Volume 2 (p. 840-844). Thousand Oaks, CA: Sage.

Day, D., \& Halpin, S. (2001). Leadership development: A review of industry best practices (Technical Report No. 1111). Fort Leavenworth, KS: Army Research Institute.

Day, D., \& O’Conner, P. (2003). Leadership development: Understanding the process. In Murphy, S. \& Riggio, R. (Eds.), The future of leadership development (pp. 11-28). Mahwah, New Jersey: Lawrence Erlbaum Associates.

Day, D., \& Halpin, S. (2004). Growing leader for tomorrow: An introduction. In Day, D., Zaccaro, S., \& Halpin, S. (Eds.), Leader development for transforming organizations: Growing leaders for tomorrow (pp. 3-22). Mahwah, New Jersey: Lawrence Erlbaum Associates.

Day, D., \& Lance, C. (2004). Understanding the development of leadership complexity through latent growth modeling. In Day, D., Zaccaro, S., \& Halpin, S. (Eds.), Leader development for transforming organizations: Growing leaders for tomorrow ( $\mathrm{p}$. 41-69). Mahwah, New Jersey: Lawrence Erlbaum Associates.

Delahoussaye, M. (2001). Leadership in the $21^{\text {st }}$ century. Training, 38 (8), 50-59.

Day, D., \& Zaccaro, S. J (2004). Toward a science of leader development. In Day, D., Zaccaro, S., \& Halpin, S. (Eds.), Leader Development for Transforming Organizations: Growing Leaders for Tomorrow (p. 383-399). Mahwah, New Jersey: Lawrence Erlbaum Associates.

De Loo, I. (2002). The troublesome relationship between action learning and organizational growth. Journal of Workplace Learning, 14 (5/6), 245-255.

Dixon, N. (1996). New routes to evaluation. Training \& Development, 50 (5), 82 85.

Doltich, D. L., \& Noel, J. L. (1998). Action learning: How the world's top companies are creating their leaders themselves. San Francisco: Jossey-Bass.

Drath, W. (2001). The deep blue sea: Rethinking the source of leadership. San Francisco: Jossey-Bass.

Edmonstone, J. (2002). Problems and projects in action learning. Industrial and Commercial Training, 34 (6/7), 287-289.

Eichinger, R. W., \& Lombardo, M. M. (2004). Knowledge summary series: 360degree assessment. Human Resource Planning, 26 (4), 34-44. 
Erikson, E. (1959). Identity and the life cycle. New York: Norton.

Facteau, J. D., Dobbins, G. H., Russell, J. E. A., Ladd, R. T. \& Kudisch, J. D. (1995). The influence of general perceptions of the training environment on pretraining motivation and perceived training transfer. Journal of Management, 21 (1), 1-24.

Fernandez, C. F., \& Vecchio, R. P. (1997). Situational leadership theory revisited: A test of an across-jobs perspective. Leadership Quarterly, 8 (1), 67-84.

Floodgate, J. \& Nixon, A. (1994). Personal development plans: The challenge of implementation - a case study. Journal of European Industrial Training, 18 (11), 43-47.

Frisch, M. H. (2001). The emerging role of the internal coach. Consulting Psychology Journal: Practice and Research, 53 (4), 240-250.

Fulmer, R. M. (1997). The evolving paradigm of leadership development. Organizational Dynamics, 25 (4), 59-72.

Fulmer, R. M., \& Conger, J. A. (2004). Identifying talent. Executive Excellence, $21(4), 11$.

Fulmer, R., \& Wagner, S. (1999). Leadership: Lessons from the best. Training \& Development, 53 (3), 29-32.

Fulmer, R., Gibbs, P., \& Goldsmith, M. (2000). Developing leaders: How winning companies keep on winning. MIT Sloan Management Review, 42 (1), $49-59$.

Garavan, T. N., Morley, M., \& Flynn, M. (1997). 360 degree feedback: Its role in employee development. Journal of Management Development, 16 (2), 134-147.

Gasper, R. (1992). Transformational leadership: An integrative review of the literature. Unpublished doctoral dissertation, Western Michigan University, Kalamazoo.

Geber, B. (1995). Does training make a difference? Prove it! Training, March, 1995, 27-34.

Giber, D., Carter, L. \& Goldsmith, M. (Eds.) (2000). Linkage Inc. 's best practices in leadership development handbook. San Francisco, CA: Jossey-Bass Pfeiffer.

Gist, M. E., Schwoerer, C., \& Rosen, B. (1989). Effects of alternative training methods on self efficacy and performance in computer software training. Journal of Applied Psychology, 74, 884-891.

Goldwasser, D. (2001). Beyond ROI. Training, 38 (1), 82-90. 
Goleman, D. (2000). Leadership that gets results. Harvard Business Review, March-April, 2000, 78-90.

Goleman, D., Boyatzis, R. \& McKee, A. (2002). Primal leadership: Realizing the power of emotional intelligence. Boston, MA: Harvard Business School Press.

Goodson, J. R., McGee, G. W., \& Cashman, J. F. (1989). Situational leadership theory: A test of leadership prescriptions. Group and Organization Studies, 14, 446-461.

Greguras, G. J., Ford, J. M., \& Brutus, S. (2002). Manager attention to multisource feedback. Journal of Management Development, 22 (4), 345-361.

Grove, J. T., Kibel, B. M. \& Hass, T. (2005). Evalulead: A guide for shaping and evaluating leadership development programs. Oakland, CA: Public Health Institute.

Hall, B. (2003). Click here for ROI. Training, 40 (3), 15.

Halpren, D. F. (2004). The development of adult cognition: Understanding constancy and change in adult learning. In Day, D., Zaccaro, S., \& Halpin, S. (Eds.), Leader development for transforming organizations: Growing leaders for tomorrow ( $\mathrm{p}$. 125-152). Mahwah, New Jersey: Lawrence Erlbaum Associates.

Harland, L. (2003). Using personality tests in leadership development: Test format effects and the mitigating impact of explanations and feedback. Human Resource Development Quarterly, 14 (3), 285-301.

Hartley, J., \& Hinksman, B. (2003). Leadership development: A systemic review of the literature. Retrieved March 5, 2005 from http://www.leadership.modern.nhs.uk

Hambleton, R. K., \& Gumpert, R. (1982). The validity of Hersey and Blanchard's theory of leader effectiveness. Group and Organization Studies, 7 (2), 225-242.

Heifetz, R. A., \& Linsky, M. (2002). Leadership on the line. Cambridge, MA: Harvard Business Review.

Hernez-Broome, G. \& Hughes, R. L. (2004). Leadership development: Past, present, and future. Human Resource Planning, 27 (1), 24-32.

Herzberg, F. (1968). One more time: How do you motivate employees? Harvard Business Review, 46 (1), 53-63.

Higson, M. \& Wilson, J. (1995). Implementing personal development plans: A model for trainers, managers and supervisors. Industrial and Commercial Training, 27 (6), 25-29. 
Hoiland, E. Brain Plasticity: What is it? Retrieved on April 6, 2005 from http://faculty.washington.edu/chudler/plast.html

Huczynski, A. A., \& Lewis, J. W. (1980). An empirical study into the learning transfer process in management training. Journal of Management Studies, 17 (2), $227-$ 240.

Hunt, J. G. (1991). Leadership: A new synthesis. Newbury Park, CA: Sage.

Imel, S. (1998). Transformative learning in adulthood (Columbus, OH: ERIC Clearinghouse on Adult Career and Vocational Education). (ERIC Document Reproduction Service No. ED423426)

Janson, R. (1970). Job enrichment. Challenge of the 70's. Training \& Development Journal, 24 (6), 7-9.

Kegan, R. (1982). The evolving self: Problem and process in human development. Cambridge, Massachusetts: Harvard.

Kegan, R. (1994). In over our heads: The mental demands of modern life. Cambridge, Massachusetts: Harvard.

Kegan, R., \& Lahey, L. (1984). Adult leadership and adult development: A constructivist view. In Kellerman, B. (Ed.), Leadership: Multidisciplinary perspectives (pp. 199-229). Englewood Cliffs, New Jersey: Prentice Hall.

Keys, L. (1994). Action learning: Executive development of choice for the 1990s. Journal of Management Development, 13 (8), 50-56.

Kiel, F., Rimmer, E., Williams, K., \& Doyle, M. (1996). Coaching at the top. Consulting Psychology Journal: Practice and Research, 48, 67-77.

Kilburg, R. R. (1996). Toward a conceptual understanding and definition of executive coaching. Consulting Psychology Journal: Practice and Research, 48 (2), 134144.

Kilburg, R. R. (2000). Executive coaching: Developing managerial wisdom in a world of chaos. Washington, DC: American Psychological Association.

Kincaid, S. B., \& Gordick, D. (2003). The return on investment of leadership development: Differentiating our discipline. Consulting Psychology Journal: Practice and Research, 55 (1), 47-57.

Kirk, J. J., Downey, B., Duckett, S., \& Woody, C. (2000). Name your career development intervention. Journal of Workplace Learning, 12 (5), 205-216. 
Kirkpatrick, D., (1994). Evaluating training programs: The four levels. San Francisco, CA: Berrett Koehler Publishers, Inc.

Klein, K. J., \& Ziegert, J. C. (2004). Leader development and change over time: A conceptual integration and exploration of research challenges. In Day, D., Zaccaro, S., \& Halpin, S. (Eds.), Leader development for transforming organizations: Growing leaders for tomorrow (pp. 359-382). Mahwah, New Jersey: Lawrence Erlbaum Associates.

Kluger, A. N., \& DeNisi, A. (1996). The effects of feedback interventions on performance. A historical review, meta-analysis, and a preliminary feedback intervention theory. Psychological Bulletin, 119 (2), 254-284.

Kokesch-Kampa, S., \& Anderson, M. Z. (2001). Executive coaching: A comprehensive review of the literature. Consulting Psychology Journal: Practice and Research, 53 (4), 40-46.

Kotter, J. (1982). The general managers. New York: The Free Press.

Krein, T., \& Weldon, K. (1994). Making a play for training evaluation. Training \& Development, 48 (4), 62-67.

Kuhnert, K., \& Lewis, P. (1987). Transactional and transformational leadership: A constructive/developmental analysis. The Academy of Management Review, 12 (4), 648-657.

Kulik, C. C., \& Kulik, J. A. (1991). Effectiveness of computer-based instruction: An updated analysis. Computers in Human Behaviors, 7, 75-94.

Lahey, L., Souvaine, E., Kegan, R., Goodman, R., \& Felix, S. (1988). A guide to the subject-object interview: Its administration and interpretation. Cambridge, MA: Subject-Object Research Group.

Laske, O. E. (1999). An integrated model of development coaching. Consulting Psychology Journal: Practice and Research, 51, 139-159.

Leobowitz, Z. B. (1987). Developing career development systems: Principles and practices. Human Resource Planning, 10 (4),195-207.

Levinson, H. (1996). Executive coaching. Consulting Psychology Journal: Practice and Research, 48 (2), 115-123.

Levinson, D. J. (1978). The seasons of a man's life. New York: Knopf.

Livers, A. B., \& Caver, K. A. (2005). Leadership development across race. In McCauley, C. D., \& Van Velsor, E. (Eds.), The center for creative leadership handbook of leadership development (p. 304-330). San Francisco: Jossey-Bass. 
London, M. (2002). Leadership development: Paths to self-insight and professional growth. Mahwah, NJ: Lawrence Erlbaum Associates.

Lowe, K., Kroeck, K., \& Sivasubramaniam, N. (1996). Effectiveness correlates of transformational and transactional leadership. Leadership Quarterly, 7 (3), 385-425.

Machiavelli, N. (1505). The Prince. Retrieved December 2, 2004 from http://www.constitution.org/mac/prince $00 . h$ tm

Martocchio, J. J. (1994). Effects of conceptions of ability on anxiety, selfefficacy, and learning in training. Journal of Applied Psychology, 79, 819-25.

Martocchio, J. J., \& Baldwin, T. T. (1997). The evolution of strategic organizational training: New objectives and research agenda. Research in Personnel and Human Resources Management, 15, 1-46.

Mauer, T. J., \& Palmer, J. K. (1999). Management development intentions following feedback: Role of perceived outcomes, social pressures, and control. The Journal of Management Development, 18 (9), 733-751.

Merriam, S. (1998). Qualitative research and case study application in education. San Francisco: Jossey-Bass.

Merriam, S. B., \& Caffarella, R. S. (1999). Learning in adulthood: A comprehensive guide. San Francisco: Jossey-Bass.

McCall, M. (1992). Executive development as a business strategy. Journal of Business Strategy, 3 (January/February), 25-31.

McCall, M. W., Lombardo, M. M., \& Morrison, A. M. (1988). The lessons of experience: How successful executives develop on the job. Lexington, MA: Lexington Books.

McCauley, C. D. (2001). Leader training and leader development. In Zaccaro, S. J., \& Klimoski, R. J. (Eds.), The nature of organizational leadership: Understanding the performance imperatives confronting today's leaders (p. 347-383). San Francisco: Jossey-Bass.

McCauley, C. D. \& Douglas, C. (1998). Developmental relationships. In McCauley, C. D., Moxley, R. S., and Van Velsor, E. (Eds.), The center for creative leadership handbook of leadership development (p. 160-193). San Francisco: JosseyBass.

McCauley, C. D. \& Van Velsor, E. (Eds.). (2005). The center for creative leadership handbook of leadership development. San Francisco: Jossey-Bass. 
McCauley, C. D. \& Moxley, R. (1996). Developmental 360: How feedback can make managers more effective. Career Development International, 1 (3), n.p.

McCauley, C. D., \& Young, D. P. (1993). Creating developmental relationships: Roles and strategies. Human Resource Management Review, 3 (3), 219-230.

McCauley, C. D., Eastman, L. L., \& Ohlott, P. J. (1995). Linking management selection and development through stretch assignments. Human Resources Management, 34 (1), 93-115.

McCauley, C. D., Moxley, R., \& Van Velsor, E. (Eds.). (1998). The center for creative leadership handbook of leadership development. San Francisco: Jossey-Bass.

McCauley, C. D., Ruderman, M. N., Ohlott, P. J., \& Morrow, J. E. (1994). Assessing the developmental components of managerial jobs. Journal of Applied Psychology, 79 (4), 544-560.

Mezirow, J. \& Associates (2000). Learning as transformation: Critical perspectives on a theory in progress. San Francisco: Jossey-Bass.

Moxley, R. \& O’Conner-Wilson, P. (1998). A systems approach to leadership development. In McCauley, C. D., Moxley, R., \& Van Velsor, E. (Eds.), The center for creative leadership handbook of leadership development (p. 217-241). San Francisco: Jossey-Bass.

Mumford, M. D., \& Manley, G. G. (2003). Putting the development in leadership development: Implications for theory and practice. In Murphy, S. \& Riggio, R. (Eds.), The future of leadership development (p. 11-28). Mahwah, New Jersey: Lawrence Erlbaum Associates.

Murphy, S. E., \& Riggio, R. E. (2003). Introduction to the future of leadership development. In Murphy, S. \& Riggio, R. (Eds.), The future of leadership development (pp. 11-28). Mahwah, New Jersey: Lawrence Erlbaum Associates.

Niemes, J. (2002). Discovering the values of executive coaching as a business transformation tool. Journal of Organizational Excellence, Autumn 2002, 61-69.

North, R. F. J., Strain, D. M. \& Abbott, L. (2000). Training teachers in computerbased management information systems. Journal of Computer-Assisted Learning, 16, 2740 .

O'Hara, J. M. (1990). The retention of skills acquired through simulator-based training. Ergonomics, 33, 1143-1153. 
Ohlott, P. (2005). Job assignments. In McCauley, C. D., \& Van Velsor, E. (Eds.), The center for creative leadership handbook of leadership development (pp. 151-182). San Francisco: Jossey-Bass.

O’Neil, H., \& Fisher, Y. (2004). A technology to support leader development: Computer games. In Day, D., Zaccaro, S., \& Halpin, S. (Eds.), Leader development for transforming organizations: Growing leaders for tomorrow (pp. 41-69). Mahwah, New Jersey: Lawrence Erlbaum Associates.

Palus, C. J., Horth, D. M., Selvin, A. M., \& Pulley, M. L. (2003). Exploration for development: Developing leadership by making shared sense of complex challenges. Consulting Psychology Journal: Practice and Research, 55 (1), 26-40.

Parker, S. K. (1998). Enhancing role breadth self efficacy: The roles of job enrichment and other organizational interventions. Journal of Applied Psychology, 83 (6), 835-852.

Parkes, D. (1998). Action learning: Business applications in North America. Journal of Workplace Learning, 10 (3), n.p.

Parry, S. (1997). Evaluating the impact of training: A collection of tools and techniques. Alexandria, VA: ASTD.

Patten, T. H. (1977). Job evaluation and job enlargement: A collision course? Human Resource Management, 16 (4), 2-8.

Patton, M. Q. (1997). Utilization-focused evaluation: The new century text. Thousand Oaks, CA: Sage.

Pernick, R. (2001). Creating a leadership development program: Nine essential tasks. Public Personnel Management, 30 (4), 429-444.

Peters, L., Hartke, D., \& Pohlman, J. (1985). Fiedler's contingency theory of leadership: An application of the meta-analysis procedure of Schmidt and Hunter. Psychological Bulletin, 97, 274-285.

Phillips, J. (1991). Handbook of training evaluation and measurement methods (2nd ed.). Houston, TX: Gulf.

Phillips, J. (Ed.) (1994). In action: Measuring return on investment. Volume I. Alexandria, VA: ASTD.

Phillips, J. (1996). How much is the training worth? Training \& Development, 50 (4), 20-24. 
Phillips, J. (Ed.) (1997). In action: Measuring return on investment. Volume II. Alexandria, VA: ASTD.

Phillips, J., \& Stone, R. (2000). Level 4 evaluation: Business results. Info Line. Alexandria, VA: ASTD.

Phillips, J., Jones, W. \& Schmidt, C. (2000). Level 3 application: Business results. Info Line. Alexandria, VA: ASTD.

Phillips, J., Wright, J., \& Pettit-Sleet, S. (2000). Level 1 evaluation: Reaction and planned action. Info Line. Alexandria, VA: ASTD.

Pierce, J. L., \& Dunham, R. B. (1976). Task design: A literature review. Academy of Management Review, 1 (4), 83-97.

Peters, J., \& Smith, P.A.C. (1998). Action learning and the leadership development challenge. Journal of Workplace Learning, 10 (6/7), 284-291.

Peterson, D. B. (1996). Executive coaching at work: The art of one-on-one change. Consulting Psychology Journal: Practice and Research, 48 (2), 78-86.

Polyani, M. (1983). The tacit dimension. Gloucester, MA: Peter Smith.

Popper, M., \& Lipshitz, R. (1993). Putting leadership theory to work: A conceptual framework for theory-based leadership development. Leadership \& Organization Development Journal, 14 (7), 23-27.

Ready, D. \& Conger, J. (2003). Why leadership-development efforts fail. MIT Sloan Management Review, 44 (3), 83-88.

Ready, D., \& Yeung, A. K. (1995). Developing leadership capabilities of Global corporations: A comparative study of eight nations. Human Resource Management, 34 (4), 529-547.

Reichardt, C. S., \& Cook, T. D. (1979). Beyond qualitative versus quantitative methods. In T. D. Cook \& C. S. Reichardt (Eds.), Qualitative and quantitative methods in evaluation research. Thousand Oaks, CA: Sage.

Reif, W. E., \& Luthans, F. (1972). Does job enrichment really pay off? California Management Review, 15 (000001), 30-37.

Ruderman, M. N. (2005). Leader development across gender. In McCauley, C. D., \& Van Velsor, E. (Eds.), The center for creative leadership handbook of leadership development (p. 271-303). San Francisco: Jossey-Bass. 
Rudestam, K. E., \& Newton, R. R. (2001). Surviving your dissertation: A comprehensive guide to content and process ( ${ }^{\text {nd }}$ Ed.). Thousand Oaks, CA: Sage.

Ryan, A. M., Brutus, S., Greguras, G. J., \& Hakel, M. D. (2000). Receptivity to assessment-based feedback for management development. Journal of Management Development, 19 (4), 252-276.

Salas, E., \& Cannon-Bowers, J. A. (2001). The science of training: A decade of progress. Annual Review of Psychology, 52, 471-499.

Schwandt, T. A. (2001). Dictionary of qualitative inquiry (2nd ed.). Thousand Oaks, CA: Sage.

Silberman, M. \& Auerbach, C. (1998). Active training: A handbook of techniques, designs, case examples, and tips (2nd ed.). San Francisco: Jossey-Bass.

Sindell, M. \& Hoang, T. (2001). Leadership Development. Info Line. Alexandria, VA: ASTD.

Smith, P. A. C., \& O'Neil, J. (2003). A review of the action learning literature 1994-2000: Part 1 - bibliography and comments. Journal of Workplace Learning, 15 (2), 63-69.

Smither, J. W., London, M., Flautt, R., Vargas, Y., \& Kucine, I. (2003). Can working with an executive coach improve multisource feedback ratings over time? A quasi-experimental field study. Personnel Psychology, 56 (1), 23-44.

Spitzer, D., \& Conway, M. (2003). Link training to your bottom line. Info Line. Alexandria, VA: ASTD.

Spreitzer, G. (2003). Leadership development in the virtual workplace. In Murphy, S. \& Riggio, R. (Eds.), The future of leadership development (pp. 71-86). Mahwah, New Jersey: Lawrence Erlbaum Associates.

Strube, M. \& Garcia, J. (1981). A meta-analytical investigation of Fiedler's contingency model of leadership effectiveness. Psychological Bulletin, 90, 307-321.

Suchman, E. A. (1967). Evaluative research. New York: Russell Sage.

Tannenbaum, I., \& Yuk1, G. (1992). Training and development in work organizations. Annual Review of Psychology, 43, 399-441.

Taylor, D. \& Edge, D. (1997). Personal development plans: Unlocking the future. Career Development International, 2 (1), 21-23.

The Virtual Leader. Retrieved on December 20, 2004 from www.simulearn.net 
Tichey, N. M., \& Devanna M. E. (1986). The transformational leader. New York: John Wiley \& Sons, Inc.

Tobias, L. L. (1996). Coaching executives. Consulting Psychology Journal: Practice and Research, 48 (2), 87-95.

Umstot, D. D., Mitchell, T. R., \& Bell, C. H. (1978). Goal setting and job enrichment: An integrated approach to job design. Academy of Management: The Academy of Management Review, 3 (000004), 867-879.

Van Velsor, E. \& Drath, W. H. (2005). A lifelong developmental perspective on leader development. In McCauley, C. D., \& Van Velsor, E. (Eds.), The center for creative leadership handbook of leadership development (p. 383-414). San Francisco: Jossey-Bass.

Van Velsor, E. \& McCauley, C. D. (2005). Introduction: Our view of leadership development. In McCauley, C. D., \& Van Velsor, E. (Eds.), The center for creative leadership handbook of leadership development (p. 1-24). San Francisco: Jossey-Bass.

Van Velsor, E., Moxley, R. S., \& Bunker, K. A. (2005). The leader development process. In McCauley, C. D., Moxley, R., \& Van Velsor, E. (Eds.). The center for creative leadership handbook of leadership development (p.204-233). San Francisco: Jossey-Bass.

Van Velsor, E., \& Guthrie, V. A. (1998). Enhancing the ability to learn from experience. In McCauley, C. D., Moxley, R., \& Van Velsor, E. (Eds.), The center for creative leadership handbook of leadership development. San Francisco: Jossey-Bass.

Vicere, A. \& Fulmer, R. (1996). Leadership by design. Boston, Massachusetts: Harvard Business School Press.

Vecchio, R. P. (1987). Situational leadership theory: An evaluation of a prescriptive theory. Journal of Applied Psychology, 72, 444-451.

Waagen, A. (1999). Essentials for evaluation. Info Line. Alexandria, VA: ASTD.

Welsh, E. T., Wanberg, C. R., Brown, K. G., \& Simmernig, M. J. (2003). Elearning: Emerging issues, empirical results and future directions. International Journal of Training and Development, 7 (4), 245-258.

When executive coaching fails to deliver (2003). Training Strategies for Tomorrow, 17 (2), 17-20.

Witherspoon, R., \& White, R. P. (1996). Executive coaching: A continuum of roles. Consulting Psychology Journal: Practice and Research, 48 (2), 124-133. 
Wright, K., Rowitz, L., \& Merkle, A. (2001). A conceptual model for leadership development. Journal of Public Health Management \& Practice, 7 (4), 60-66.

Yukl, G. (2002). Leadership in organizations (5th ed.). Upper Saddle River, NJ: Prentice Hall.

Zacharatos, A., Barling, J., \& Kelloway, E. (2000). Development and effects of transformational leadership in adolescents. Leadership Quarterly, 11 (2), 211-226.

Zemke, R. (1992). Second thoughts about the MBTI. Training, 29 (4), 43-47.

Zenger, J., Ulrich, D., \& Smallwood, N. (2000). The new leadership development. Training \& Development, 54 (3), 22-27.

Zheng, Y. \& Kleiner, B. H. (2001). Developments concerning career development in transitions. Management Research News, 24 (3/4), 33-39.

Zuber-Skeritt, O. (2002). The concept of action learning. The Learning Organization, 9 (3), 114-124. 


\section{APPENDIX A}

\section{Mapping Leadership Development}

\begin{tabular}{|c|c|}
\hline Leadership Development Defined & $\begin{array}{l}\text { e.g., Avolio, 2004; Avolio, 2005; Davis, 2001; } \\
\text { Drath, 2001; London, 2002; Day, 2001; Day, 2004; } \\
\text { Popper \& Lipshitz, 1993; McCall, Lombardo, \& } \\
\text { Morrison, 1988; McCauley, Moxley \& Van Velsor, } \\
\text { 1998; O’Neil \& Fisher, 2004; Sindell \& Hoang, } \\
\text { 2001; and Vicere \& Fulmer, } 1996\end{array}$ \\
\hline Leadership Development Models & $\begin{array}{l}\text { e.g., Avolio, 1999; Avolio, 2005; Hunt, 1991; Klein } \\
\text { and Ziegert, 2004; Palus, Horth, Selvin \& Pulley, } \\
\text { 2003; Van Veslor, McCauley \& Moxley, 1998; } \\
\text { Vicere \& Fulmer, 1998; and Yukl, 2002. }\end{array}$ \\
\hline $\begin{array}{l}\text { Leadership Development \& Adult } \\
\text { Development Theory }\end{array}$ & $\begin{array}{l}\text { e.g., Avolio, 1999; Avolio, 2005; Avolio \& } \\
\text { Gibbons, 1989; Bass, 1990; Day \& Lance, 2004; } \\
\text { Day \& O’Conner, 2004; Day \& Zaccaro, 2004; } \\
\text { Heifetz \& Linsky, 2002; Klein, \& Ziegert, 2004; } \\
\text { Mumford \& Manley, 2003; Van Velsor \& Drath, } \\
2005 .\end{array}$ \\
\hline $\begin{array}{l}\text { Leadership Development \& Adult } \\
\text { Learning Theory }\end{array}$ & $\begin{array}{l}\text { e.g., Avolio, 1999; Conger \& Benjamin, 1999; } \\
\text { Goleman, Boyatzis \& McKee, 2002; Halpren, 2005; } \\
\text { London, 2002; Murphy \& Riggio, 2003; and } \\
\text { Wright, Rowitz, \& Merkle, 2001. }\end{array}$ \\
\hline $\begin{array}{l}\text { Leadership Development \& } \\
\text { Organizational Context }\end{array}$ & $\begin{array}{l}\text { e.g., Avolio, 1999; Avolio, 2005; Bass, 1990; } \\
\text { Cacioppe, 1998; Conger, 1989; Conger \& } \\
\text { Benjamin, 1999; Davis, 2001; Day, 2001; Fulmer \& } \\
\text { Wagner, 1999; Fulmer, Gibbs, \& Goldsmith, 2000; } \\
\text { Giber, Carter, \& Goldsmith, 2000; Goleman et al., } \\
\text { 2002; Hernez-Bloom \& Hughes, 2004; Klein, \& } \\
\text { Ziegert, 2004; London, 2002; McCauley, 2001; } \\
\text { McCauley, Moxley, \& Van Velsor, 1998; Pernick, } \\
\text { 2001; Ready \& Conger, 2003; Ready, \& Yeung, } \\
\text { 1995; Tichey \& Devanna, 1986; Vicere \& Fulmer, } \\
\text { 1996; and Yukl, 2002. }\end{array}$ \\
\hline $\begin{array}{l}\text { Leadership Development Rooted in } \\
\text { Leadership Theory }\end{array}$ & $\begin{array}{l}\text { e.g., Avolio, 1999; Avolio, 2005; Cacioppe, 1998; } \\
\text { Conger, 1992; Conger \& Benjamin, 1999; Goleman, } \\
\text { Boyatzis \& McKee, 2002; Popper \& Lipshitz, 1993; } \\
\text { and Vicere \& Fulmer, 1998. }\end{array}$ \\
\hline Leadership Development \& Time & $\begin{array}{l}\text { e.g., Avolio, 1999; Avolio \& Gibbons, 1989; Avolio } \\
\text { 2005; Conger \& Benjamin, 1999; and Fulmer, } 1997 .\end{array}$ \\
\hline Leadership Development Tools & $\begin{array}{l}\text { e.g., Bass, 1990; Cacioppe, 1998; Conger \& } \\
\text { Benjamin, 1999; Day, 2001; Day, 2004; Day \& } \\
\text { Halpin, 2001; Day \& Zaccaro, 2004; Hartley \& } \\
\text { Hinksman, 2003; Hunt, 1991; Giber, Carter, \& } \\
\text { Goldsmith, 2000; Klein, \& Ziegert, 2004; London, } \\
\text { 2002; McCauley, 2001; Yukl, 2002; Van Veslor, } \\
\text { McCauley \& Moxley, 1998; Vicere \& Fulmer, } \\
\text { 1998; and Zenger, Ulrich, \& Smallwood, 2000. }\end{array}$ \\
\hline
\end{tabular}




\begin{tabular}{|c|c|}
\hline The Process of Leadership Development & $\begin{array}{l}\text { e.g., Cacioppe, 1998; Giber, Carter, \& Goldsmith, } \\
\text { 2000; London, 2002; Van Velsor, Moxley \& } \\
\text { Bunker, 2005; and Vicere \& Fulmer, } 1998\end{array}$ \\
\hline $\begin{array}{l}\text { Curriculum Content of a Leadership } \\
\text { Development Process } \\
\text { Curriculum Content of a Leadership } \\
\text { Development Process }\end{array}$ & $\begin{array}{l}\text { e.g., Avolio, 1999; Avolio, 2005; Cacioppe, 1998; } \\
\text { Conger, 1992; Conger, 1993; Day, 2001; Day \& } \\
\text { O’Conner, 2003; Day \& Halpin, 2004; Giber, Carter } \\
\text { \& Goldsmith, 2000; Goleman, Boyatzis \& McKee, } \\
\text { 2002; Hunt, 1991; London, 1999; Popper \& } \\
\text { Lipshitz, 1993; Van Veslor, McCauley \& Moxley, } \\
\text { 1998;and Yukl, 2002. }\end{array}$ \\
\hline Leadership Development \& Context & $\begin{array}{l}\text { e.g., Day \& Lance, 2004; London, 1999; Goleman, } \\
\text { et al., 2002; Hartley \& Hinksman, 2003; and } \\
\text { Zenger, Ulrich, \& Smallwood, } 2000 .\end{array}$ \\
\hline Evaluating Leadership Development & $\begin{array}{l}\text { e.g., Avolio, 2005; Cacioppe, 1998; Conger, 1993a; } \\
\text { Day, 2004; Day \& O’Conner, 2004; Fulmer \& } \\
\text { Wagner, 1999; Fulmer, Gibbs, \& Goldsmith, 2000; } \\
\text { Giber, Carter, \& Goldsmith, 2000; Hartley \& } \\
\text { Hinksman, 2003; Hernez-Broome \& Hughes, 2004; } \\
\text { Kincaid \& Gordick, 2003; London, 2002; } \\
\text { McCauley, Moxley, \& Van Velsor, 1998; Ready \& } \\
\text { Conger, 2003; Wright, Rowitz, \& Merkle, 2001; } \\
\text { and Yukl, 2002. }\end{array}$ \\
\hline Leadership Development \& Gender & e.g., Ruderman, 2005. \\
\hline Leadership Development \& Race & e.g., Livers \& Caver, 2005. \\
\hline Leadership Development \& Technology & $\begin{array}{l}\text { e.g., Avolio, 2005; Avolio \& Kahai, 2003; O’Neil \& } \\
\text { Fisher, 2005; and Spreitzer, } 2003 .\end{array}$ \\
\hline Trends in Leadership Development & e.g., London, 2002 \\
\hline $\begin{array}{l}\text { Levels of Leadership Development } \\
\text { (Leader/Leadership Development) }\end{array}$ & $\begin{array}{l}\text { e.g., Avolio, 2005; Day, 2001; Day, 2004; and } \\
\text { McCauley \& Van Velsor, } 2005\end{array}$ \\
\hline $\begin{array}{l}\text { Types of Leadership Development } \\
\text { Programs }\end{array}$ & e.g., Conger, 1992 \\
\hline
\end{tabular}




\section{APPENDIX B}

Alpha Company

"Lucy's" Theory of Action

\section{Program Objectives}

\section{Ultimate Objectives}

Focus on long-term effects - results and lasting behavior change

7. A Culture of Engagement vs. Compliance

Intermediate Objectives

Focus on short-term outcomes - participants, reactions and knowledge, skill \& attitude changes.

6. Succession planning may be linked to development.

5. Action plans and development plans are linked and discussed at an individual's performance appraisal.

4. "Reaction" evaluations are collected and distributed to participants for feedback.

Immediate Objectives (short term)

Focus on implementation - activities and inputs

3. Supervisors and managers from the three service lines voluntarily participate in various courses that include-classroom training instruments, action learning, external learning development plans, learning management system and books and videos.

2. Assessments are conducted to determine course curriculum.

1. Management/Development education is offered to front-line supervisors, and branch managers for the three primary service lines - corporate, operations \& sales.

\section{Validity Assumptions}

- "Alpha Co." University is working under the correct paradigm or philosophy.

- There is a commitment to level three evaluation to see if results are being achieved.

- CEO and senior leaders are supportive of a culture shift of engagement vs. compliance.

- $\quad \mathrm{CEO}$ and senior leaders model culture shift.

- Participants are willing to engage in holding themselves accountable for their own learning.

- What was learned is being reinforced by the participant's supervisor.

- Members of the management team at all levels is playing on the same team and not out for only themselves.

- Learning is being applied. 


\section{"Lucy" - Alpha Company Program Theory of Events (Theory of Action)}

1. Program Inputs

2. Program Activities

3. Program Participants

4. Reactions
Resources expended; number and type staff involved time expended.

a. Five, full- time staff "management

developers" spent a projected 2832 hours in the classroom with participants.

Implementation data on what the program actually offers or does.

a. Various programs offer classroom training, instruments, action learning, external learning opportunities, individual development plans (action plans), a learning management system, books and videos, blended learning, online materials, job rotation, mentoring and internal conferences.

Characteristics of program participants and clients; numbers, nature of involvement, background

a. First line supervisors, branch managers, regional managers, voluntary involvement

What participants and clients say about the program; satisfaction; interest, strengths and weaknesses

a. Reactions to programming is positive.

Measures of individual and group change in knowledge, attitudes and skills

a. Not measured. 
6. Practice and Behavior $\Delta \quad$ Measures of adoption of new practices and behavior over time

a. $\quad$ Not measured with the exception of one 45day follow-up discussion for one course

7. End Results

Measures of effect on overall problem, ultimate goals, side effects, social and economic consequences.

a. A culture of engagement vs. compliance 


\section{Alpha Company}

"Sandy's" Theory of Action

\section{Program Objectives}

\section{Validity Assumptions}

\section{Ultimate Objectives}

Focus on long-term effect - results and lasting behavior change

7. Not overtly suggested, but Sandy feels it is to develop manager/leaders, aid in accountability, collaboration, and foster productive conflict.

Intermediate Objectives

Short-term outcomes - participants, reactions and knowledge, skill \& attitude changes.

6. Participation is informally linked to succession planning.

5. Action plans and development plans are linked to the formal evaluation process.

4. "Reaction" evaluations are collected and distributed to participants for feedback. Additional methods of evaluation include observation, participant feedback, instructor feedback, sidebar conversations and dialogue in the classroom.

Immediate Objectives (short term)

Focus on implementation - activities and inputs

3. Supervisors and managers are heavily encouraged to participate in various courses that include classroom training, instruments, action learning, external learning opportunities, individual development plans, learning management system, books and videos, job rotation, and mentoring.

2. Based upon participant feedback, business strategy, and job descriptions, curriculum is designed.

1. Management/development education is offered to front-line supervisors, and branch managers for the three primary service lines - operations, sales and corporate. 


\section{"Sandy" - Alpha Company Program Theory of Events (Theory of Action)}

1. Program Inputs

2. Program Activities

3. Program Participants

4. Reactions

5. KSAs $\Delta$
Resources expended; number and type staff involved, time expended.

a. Five, full-time staff "management developers" staff spent a projected 2832 hours in the classroom with participants.

Implementation data on what the program actually offers or does.

a. Various programs offer classroom training, instruments, action learning, external learning opportunities, individual development plans, learning management system, books and videos, job rotation, and mentoring.

The characteristics of program participants and clients; numbers, nature of involvement, background

a. First-line supervisors, branch managers, regional managers, voluntary involvement (although it is heavily encouraged)

What participants and clients say about the program; satisfaction; interest, strengths and weaknesses

a. Reactions to programming is positive.

Measures of individual and group change in knowledge, attitudes and skills

a. Not measured. 
6. Practice and Behavior $\Delta \quad$ Measures of adoption of new practices and behavior over time

a. Performance evaluation is formally linked to development plans and succession planning and one 45-day follow-up discussion for one course.

7. $\quad$ End Results

Measures of effect on overall problem, ultimate goals, side effects, social and economic consequences.

a. Develop manager/leaders, accountability, collaboration and productive conflict 
The Alpha Company

"Peter's" Theory of Action

\section{Program Objectives}

Ultimate Objectives

Focus on long-term impacts - end results and lasting behavior change

\section{No}

Intermediate Objectives

Focus on short term outcomes - participants, reactions and knowledge, skill \& attitude changes.

5. Action plans and development plans may be linked and discussed at an individual's performance appraisal.

4. "Reaction" evaluations are collected and distributed to participants for feedback.

Immediate Objectives (short-term)

Focus on implementation - activities and inputs

3. Supervisors and managers from the three service lines voluntarily participate in various courses that include classroom training, instruments, action learning, external learning opportunities, individual development plans (action plans), a learning management system, books and videos, blended learning, online materials, job rotation, mentoring and internal conferences.

2. Based upon the thoughts of vice presidents, participants, supervisors and the "management developers," classroom-based training curriculum and activities are designed.

1. Management/development education is offered to front-line supervisors, and branch managers for the three primary service lines - operations, sales, and corporate.

\section{Validity Assumptions}

- Management is involved and supportive in participant learning on an ongoing basis.

- Participants are prioritizing what they have learned upon their return.

- Training is ongoing once participants return.

- Participants are legitimately and proactively involved in their own development and not led by the nose.

- What was learned is being reinforced.

- They will execute their action plans.

- The training was effective.

- What we are teaching is of value to participants.

- $\quad$ supervisors and managers make every effort to come. None of the management training is mandatory.

- We are teaching appropriate materials 


\section{"Peter" - Alpha Company Program Theory of Events (Theory of Action)}

1. Program Inputs

2. Program Activities

3. Program Participants

4. Reactions

5. $\quad$ KSAs $\Delta$
Resources expended; number and type staff involved time expended.

a. Five, full-time staff “management developers" spent a projected 2832 hours in the classroom with participants.

Implementation data on what the program actually offers or does.

a. Various programs offer classroom training, instruments, action learning, external learning opportunities, individual development plans (action plans), a learning management system, books and videos.

The characteristics of program participants and clients; numbers, nature of involvement, background

a. First-line supervisors, branch managers, regional managers, voluntary involvement

What participants and clients say about the program; satisfaction; interest, strengths and weaknesses

a. Reactions to programming is positive.

Measures of individual and group change in knowledge, attitudes and skills

a. Not measured. 
6. Practice and Behavior $\Delta \quad$ Measures of adoption of new practices and behavior over time

a. Not measured with the exception of one 45-day follow-up discussion for one course

7. End Results

Measures of effect on overall problem, ultimate goals, side effects, social and economic consequences.

a. Not defined and/or measured. 


\section{APPENDIX C}

\section{Informed Consent Antioch University PhD in Leadership \& Change}

This study examines how academic notions of leadership development compare and contrast with the theory of action that guides corporate leadership development programs. It is performed as partial fulfillment of the requirements for the researcher's (Scott Allen) Ph.D. in Leadership and Change at Antioch University.

As a participant in this study, the researcher asks you to engage in four interviews related to the leadership development program in your organization.

- Meeting one - In meeting one, the focus will be to establish rapport, understand your role in the organization, your role in relation to the leadership development program, the organization's structure, and a broad overview of the leadership development program.

- Meeting two - In meeting two, I will confirm information gathered from meeting one and closely examine the leadership development program.

- Meeting three - In meeting three, I will confirm information from previous meetings and begin discussing validity assumptions based upon the theory of action developed in meetings one and two.

- Meeting four - In meeting four, I will meet with all three participants together to discuss the overall theory of action. Because there will be differences in responses, this meeting will align the three perspectives into one theory of action and corresponding validity assumptions.

The interviews will be about 1.5 hours in length and there will be four interviews in all. The interviews will take place over two months (at your convenience) and will be tape recorded for later analysis by the researcher. You will have an opportunity in interviews 2-4 to review the researcher's understanding of your ideas as they emerged in previous interviews. At the conclusion of the research process, the researcher will be available to each participant to discuss the overall findings of the study. If any quotations from the interviews are used in the final summary, you as the interviewee will be asked to approve their inclusion. 
There are no foreseeable risks with this research. However, all subjects should understand that their responses will be shared with the group in meeting four. At this point, their confidentiality will not be ensured and all three participants will know what the others reported.

The main potential benefit is in contributing to scientific knowledge on leadership development. No costs or payments are associated with participating in the study. If you have any questions about the nature and purpose of this research, the researcher will be happy to answer your questions prior to the beginning of our interview. If at anytime during the interview you feel uncomfortable, you may stop the process and terminate your participation in the study.

I agree to participate in this research project and I understand that

1. my participation is entirely voluntary. I may terminate my participation at any time without penalty.

2. all tape recordings will be destroyed after completion of the study

3. if I have questions about the research or, if I would like a copy of the aggregate findings of the study when it is complete, I can contact the researcher by calling 216.224 .7072 or sallen@phd.antioch.edu or the supervising faculty member, Professor Jon Wergin at jwergin@phd.antioch.edu.

Signed Date

(Participant)

Signed Date

(Scott Allen, Student Researcher) 